\title{
Investigation of the diachronic development of $\mathrm{CO}_{2}$ emission levels in relation to traffic characteristics along major road axes during an era of recession: The case of Greece
}

\author{
Pichou S., Tsakalidis A. and Kehagia F.* \\ Department of Civil Engineering, Aristotle University of Thessaloniki, AUTH Campus, 54124, Thessaloniki, Greece \\ Received: 19/06/2017, Accepted: 12/10/2017, Available online: 28/11/2017 \\ *to whom all correspondence should be addressed: e-mail: fkehagia@civil.auth.gr
}

\begin{abstract}
The problem of reducing $\mathrm{CO}_{2}$ emissions from transport, a major contributor to the greenhouse effect, has become a growing concern for the scientific community and various international committees monitoring climate change. Energy savings in the transport sector are a key factor towards rational management of oil reserves, while new trends in the automotive market have already been established, supported by research on efficient and environmentally-friendly technologies and alternative fuels to face fossil fuel dependency. The road transport sector is an important part for most developed economies but also a major source of pollutant emissions. In this framework, this paper focuses on transport emissions along the main road axis in Greece, connecting the country's two largest urban areas, during the years 20082014, a period of prolonged recession. Based on traffic data collected at the toll stations along the highway, greenhouse gas and pollutant emissions were calculated using the COPERT4 emission estimation tool. According to the results, a sharp fall in emissions is observed largely due to traffic volume reductions, but also due to a prevailing trend for larger displacement vehicles and technologically improved vehicles with better environmental standards.
\end{abstract}

Keywords: $\mathrm{CO}_{2}$ Emissions, Transport System, Road infrastructure, Road Transport, Traffic Characteristics, Recession.

\section{Introduction}

The transport sector, one of the fastest growing sectors of human activity, is a key economic and productivity factor of any society, although it progressively led to evident environmental impacts and to the appearance of harmful effects on human health. In the European Union (EU), passenger and freight transport increased considerably until 2008. In the years following the economic crisis beginning in 2008, the passenger transport has remained stable, while in the freight transport demand a sharp fall has occurred. The transport sector is the only main European economic sector in which greenhouse gas (GHG) emissions have increased since 1990 by estimated $19.4 \%$, whereas all other sectors have reduced their emissions. It should be noted that in 2013, transport was responsible for $25 \%$ of total GHG emissions in Europe. Road transport was responsible for almost $73 \%$ of all GHG transport emissions. Passenger cars accounted for $60 \%$ of all road transport emissions and heavy-duty vehicles for 27\% (European Commission, 2015; European Environment Agency, 2015).

Regarding the total GHG emissions in the EU, a decrease by 22.9\% was observed between 1990 and 2014 (in Greece the decline was $2.8 \%$ ), and by $17.9 \%$ between 2004 and 2014. However, total GHG emissions, deriving only from the transport sector, increased by $13.3 \%$ between 1990 and 2014, but decreased by $8.5 \%$ from 2004 to 2014 . For $\mathrm{CO}_{2}$, which is the most important GHG (it holds approximately $80 \%$ worldwide) and a key component of the exhaust gases, it was observed that emissions decreased by $20.6 \%$ from 1990 to 2014 and by $18.9 \%$ between 2004 and 2014. On the contrary, $\mathrm{CO}_{2}$ emissions from the transport sector, increased by $24.1 \%$ from 1990 to 2014 , but decreased by $8.4 \%$ between 2004 and 2014 . Similarly, the respective percentages for Greece concerning transport $\mathrm{CO}_{2}$ emissions, increased by $22.2 \%$ between 1990 and 2014 and decreased by $18.1 \%$ between 2004 and 2014 (European Environment Agency, 2016).

Moreover, apart from GHG, transport contributes significantly to the emissions of many air pollutants. Significant progress has been made since 1990 in reducing the emissions of many air pollutants due to the introduction of the Euro emission standards for vehicles and the gradual fleet renewal (European Environment Agency, 2015).

As mentioned above, road traffic is the greatest contributor to the transport sector carbon footprint and, therefore, its reduction has become one of the main targets of sustainable transport policies. An analysis of the main factors influencing GHG emissions is essential for designing new energy and environmentally efficient road transport strategies. General, physical, economic and social factors influence transport systems' development (Enoch et al., 2004), while the current global financial and economic crisis is affecting transport infrastructure projects (Wang, 2009). Few studies have been focused on 
the connections between urban form, location effects and transport behavior under changing economic conditions (Nielsen, 2015). Efthymiou and Antoniou (2017) investigated the impact of crisis on public transport users' satisfaction and demand, while Lee (2010), Papagiannakis et al., (2017) and Sobrino (2014) addressed the questions of how travel behavior is affected by the economic recession and what it would mean towards the goal of achieving sustainable transport. Lyamani et al. (2011) presented that the reduction in fossil fuels use due to the economic slowdown contributed significantly to the observed decrease in black carbon concentrations in 2008.

Within the latest global financial crisis context, which had a strong impact also on many EU countries since 2008, the transport sector was also affected, since it is one of the main pillars of developed economies. Among the affected EU countries, Greece suffered the most severe and prolonged recession, with side effects on all sectors of the economy, including transport. In May 2010, the International Monetary Fund (IMF) along with Eurozone governments provided Greece short and medium-term loans of more than 100 billion euros. In order to ensure repayment, the Greek government announced spending cuts; the latter including major reductions in public transport investment and subsidies. In addition, industry, all commercial activities and services have been severely affected by recession and are significantly limited following overall economy trends (Christoforou and Karlaftis, 2011).

In this framework, this paper focuses on the Greek economic recession influence on exhaust emissions from transport, using as a case study the traffic and emissions effects observed along the main highway infrastructure in Greece i.e. Highway A1, which connects the country's two largest urban metropolitan areas, Athens and Thessaloniki, during the recession period of 2008-2014.

\section{Materials and Methods}

The methodology for the analysis of the environmental performance of roadway infrastructure was based on the following steps:

1. traffic and economic data collection

2. traffic volume analysis

3. traffic emission estimation using COPERT

\subsection{Traffic and economic data collection}

According to the registered vehicles database of the Hellenic Statistical Authority (2017), since 1985 the number of total motor vehicles has been almost fourfold in Greece (Table 1). Private cars in Greece reached over 5 million in 2015. During the decade 2000-2010 an annual increase by about $5 \%$ was observed while the total increase in this period was $60 \%$. However, since 2011 , an annual decrease, in the total vehicles, by about $0.3 \%$ has been noticed due to economic crisis. Moreover, the number of motorcycles is increasing by about $1.5 \%$ annually.

According to the statistics of the European Automobile Manufacturers' Association (2016), a stable annual increase of passenger cars per 1000 inhabitants has been observed and almost one car corresponds to every two citizens. In Greece a stable status has been observed since 2010 as presented in Table 2.

Table 1. Motor vehicle in operation per category in Greece (1985-2015)

\begin{tabular}{cccccccc}
\hline & $\mathbf{1 9 8 5}$ & $\mathbf{1 9 9 0}$ & $\mathbf{1 9 9 5}$ & $\mathbf{2 0 0 0}$ & $\mathbf{2 0 0 5}$ & $\mathbf{2 0 1 0}$ & $\mathbf{2 0 1 5}$ \\
\hline Passenger cars & 1259335 & 1735523 & 2204761 & 3195065 & 4303129 & 5216873 & 5107620 \\
\hline Buses & 19234 & 21430 & 24600 & 27037 & 26829 & 27311 & 26586 \\
\hline Heavy trucks & 595761 & 766429 & 883823 & 1057422 & 1186483 & 1318768 & 1322604 \\
\hline Motorcycles & 162295 & 256594 & 475668 & 781361 & 1124172 & 1499133 & 1619621 \\
\hline Total vehicles & 2036625 & 2779976 & 3588852 & 5060885 & 6640613 & 8086910 & 8076431 \\
\hline
\end{tabular}

Table 2. Number of passenger cars per 1000 inhabitants

\begin{tabular}{ccccccccccc}
\hline & $\mathbf{2 0 0 5}$ & $\mathbf{2 0 0 6}$ & $\mathbf{2 0 0 7}$ & $\mathbf{2 0 0 8}$ & $\mathbf{2 0 0 9}$ & $\mathbf{2 0 1 0}$ & $\mathbf{2 0 1 1}$ & $\mathbf{2 0 1 2}$ & $\mathbf{2 0 1 3}$ & $\mathbf{2 0 1 4}$ \\
\hline European Union & 462 & 469 & 467 & 474 & 476 & 481 & 485 & 490 & 494 & 498 \\
\hline Greece & 392 & 413 & 435 & 454 & 463 & 469 & 468 & 466 & 466 & 468 \\
\hline
\end{tabular}

The age of the car fleet within the EU is increasing; specifically, in 2014, passenger cars in Europe had an average age of 9.73 years. The same phenomenon occurs in Greece, since relevant data show that in $2014,68 \%$ of passenger cars had an age of more than 10 years (Table 3 ). There is therefore significant potential for renewing the private car fleet in Greece and for penetration of improved and more efficient engines, in a market where petrol is by far the dominant fuel type (Table 4).
Furthermore, the monitoring of the Gross Domestic Product (GDP) and its comparison with the number of the total private cars in circulation per 1000 inhabitants is of interest (Figure 1). According to data from the Hellenic Statistical Authority (2017b), from 2008 onwards, GDP dropped significantly as a result of the economic recession, while the number of registered vehicles remains broadly stable.

Table 3. Passenger car fleet by age in Greece

\begin{tabular}{crr}
\hline Age (Years) & \multicolumn{2}{c}{ Fleet share (as of 2014) } \\
\hline$>5$ & $7 \%$ & $25 \%$ \\
\hline $5-10$ & $68 \%$ & $25 \%$ \\
\hline$>10$ & & 6 \\
\hline
\end{tabular}


Table 4. Passenger car fleet by fuel type

\begin{tabular}{lccc}
\hline & Petrol & Diesel & Alternative fuels \\
\hline European Union & $54.10 \%$ & $40.97 \%$ & $4.93 \%$ \\
\hline Greece & $95.00 \%$ & $3.95 \%$ & $1.05 \%$ \\
\hline
\end{tabular}

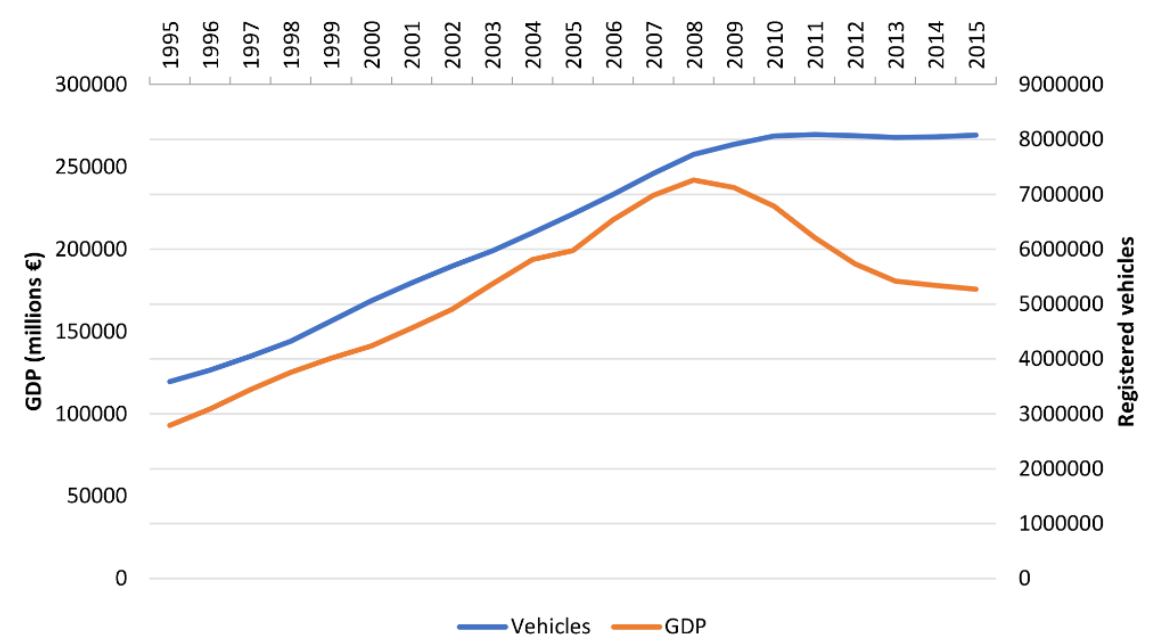

Figure 1. Evolution of the number of registered vehicles and GDP (mil. €) in Greece

For this case study, site-specific traffic data were collected from toll stations along the Highway $\mathrm{A} 1$ and analyzed for the recession period between 2008 and 2014. Highway A1, part of the European route E75, is the main north-south road axis in Greece and the country's second longest highway. It is a closed dual highway with a central reserve of total length of $495 \mathrm{~km}$, with two traffic lanes plus an emergency lane per direction for $343 \mathrm{~km}$ (total paved width of $20.4 \mathrm{~m}$ ) and three traffic lanes plus an emergency lane per direction for $152 \mathrm{~km}$ (total paved width of $27.5 \mathrm{~m}$ ).

\subsection{Traffic volume analysis}

The traffic volume of passenger and heavy vehicles measured at a series of toll stations along Highway A1 was examined for a seven-year period (2008-2014) for both directions (to Athens and to Thessaloniki) and they are presented in Table 5. The diachronic evolution of the aforementioned data of traffic volume per toll station is presented in Figures 2 and 3.

A significant downward trend in vehicle movement (passenger and heavy) is observed, particularly from 2010 onwards, coinciding with the onset of the economic crisis in Greece which resulted in fewer vehicle-kilometers travelled. It is also evident that there is a great difference in the number of vehicles between Afidnes and the other toll stations, which can be justified by the fact that Afidnes toll station is the one nearest to the capital, serving more vehicles that move daily towards both directions (either to or from Athens). The average reduction in total passenger vehicles between 2008 and 2014 for all stations is 47\%, while the average reduction of heavy vehicles is $40 \%$ respectively.

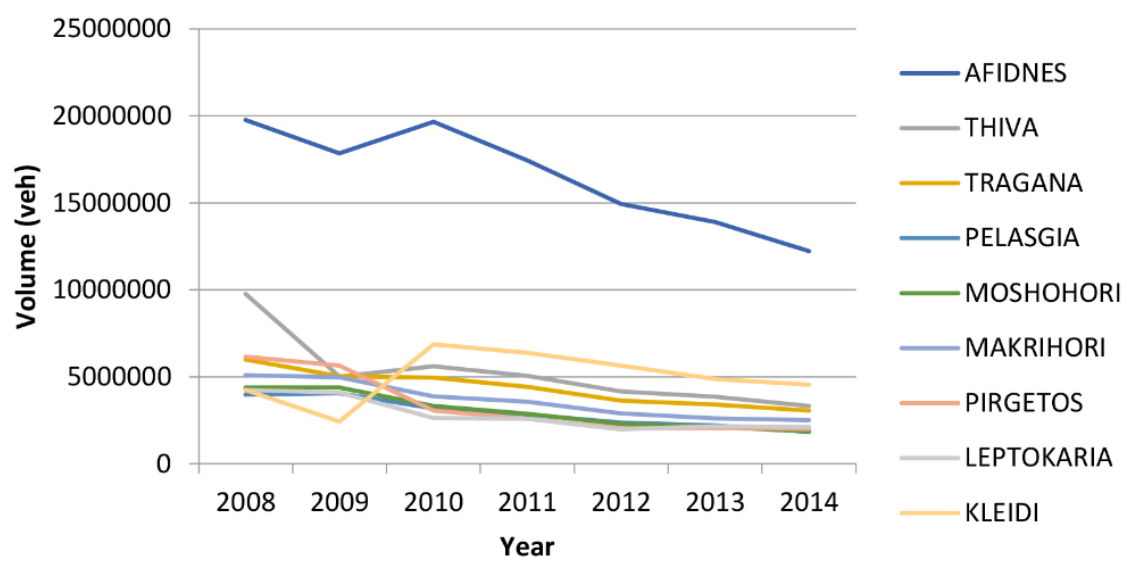

Figure 2. Total traffic volume of passenger vehicles per toll station for both directions (2008-2014) 


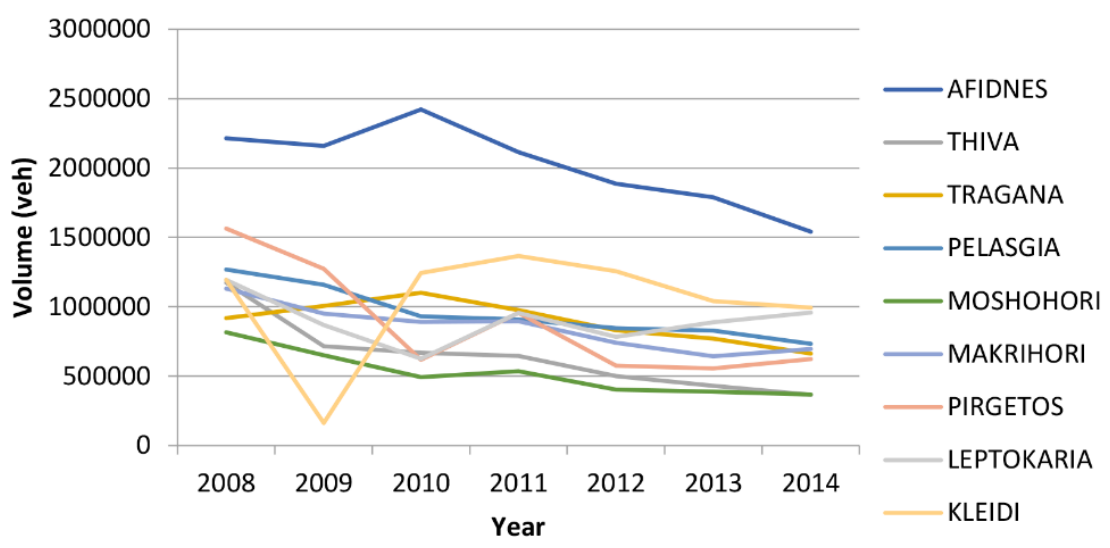

Figure 3. Total traffic volume of heavy vehicles per toll-station for both directions (2008-2014)

Table 5. Traffic volume and percentage of heavy vehicles per toll station in both directions

\begin{tabular}{|c|c|c|c|c|c|c|c|}
\hline \multirow{2}{*}{ Toll station } & \multicolumn{6}{|c|}{ Year } & \multirow[b]{2}{*}{2014} \\
\hline & 2008 & 2009 & 2010 & 2011 & 2012 & 2013 & \\
\hline \multicolumn{8}{|l|}{ ATHENS } \\
\hline \multirow{3}{*}{ (1) - AFIDNES } & $19769426^{*}$ & 17841997 & 19670388 & 17446377 & 14941327 & 13894715 & 12236176 \\
\hline & $2214726^{* *}$ & 2160028 & 2422541 & 2114274 & 1886194 & 1788862 & 1541913 \\
\hline & $10.07 \%^{* * *}$ & $10.8 \%$ & $10.97 \%$ & $10.81 \%$ & $11.21 \%$ & $11.41 \%$ & $11.19 \%$ \\
\hline \multirow{3}{*}{ (2) - THIVA } & 9767432 & 5026218 & 5616023 & 5062078 & 4172065 & 3858145 & 3334519 \\
\hline & 1174337 & 716613 & 667883 & 644430 & 499548 & 431211 & 365462 \\
\hline & $10.73 \%$ & $12.48 \%$ & $10.63 \%$ & $11.29 \%$ & $10.69 \%$ & $10.05 \%$ & $9.88 \%$ \\
\hline \multirow{3}{*}{ (3) - TRAGANA } & 6005264 & 5042789 & 4950053 & 4429986 & 3626028 & 3399869 & 3071746 \\
\hline & 919188 & 1006614 & 1101758 & 975306 & 831460 & 771842 & 662900 \\
\hline & $13.27 \%$ & $16.64 \%$ & $18.21 \%$ & $18.04 \%$ & $18.65 \%$ & $18.50 \%$ & $17.75 \%$ \\
\hline \multirow{3}{*}{ (4) - PELASGIA } & 3976327 & 4062554 & 3193313 & 2846516 & 2369426 & 2205496 & 1836577 \\
\hline & 1268935 & 1159460 & 929968 & 908563 & 845174 & 828383 & 733258 \\
\hline & $24.19 \%$ & $22.2 \%$ & $22.55 \%$ & $24.20 \%$ & $26.29 \%$ & $27.30 \%$ & $28.53 \%$ \\
\hline \multirow{3}{*}{ (5) - MOSHOHORI } & 4383949 & 4377501 & 3336892 & 2880914 & 2287269 & 2118940 & 1862119 \\
\hline & 816509 & 649580 & 494039 & 535954 & 403259 & 388895 & 367986 \\
\hline & $15.7 \%$ & $12.92 \%$ & $12.9 \%$ & $15.69 \%$ & $14.99 \%$ & $15.51 \%$ & $16.50 \%$ \\
\hline \multirow{3}{*}{ (6) - MAKRIHORI } & 5094984 & 4984553 & 3875508 & 3568038 & 2907798 & 2606526 & 2503601 \\
\hline & 1132006 & 950537 & 890484 & 895912 & 741261 & 642482 & 695980 \\
\hline & $18.18 \%$ & $16.02 \%$ & $18.68 \%$ & $20.07 \%$ & $20.31 \%$ & $19.77 \%$ & $21.75 \%$ \\
\hline \multirow{3}{*}{ (7) - PIRGETOS } & 6159190 & 5657412 & 3052629 & 2584215 & 2038056 & 2067093 & 2000164 \\
\hline & 1564174 & 1272671 & 617561 & 956780 & 575640 & 556519 & 623836 \\
\hline & $20.25 \%$ & $18.36 \%$ & $16.83 \%$ & $27.02 \%$ & $22.02 \%$ & $21.21 \%$ & $23.77 \%$ \\
\hline \multirow{3}{*}{ (8) - LEPTOKARIA } & 4251875 & 4099133 & 2632484 & 2585353 & 1973142 & 2156876 & 2134940 \\
\hline & 1194676 & 867370 & 625572 & 956282 & 781950 & 889124 & 958278 \\
\hline & $21.93 \%$ & $17.46 \%$ & $19.20 \%$ & $27 \%$ & $28.38 \%$ & $29.19 \%$ & $30.98 \%$ \\
\hline \multirow{3}{*}{ (9) - KLEIDI } & 4251875 & 2423676 & 6870224 & 6392521 & 5641346 & 4882740 & 4558138 \\
\hline & 1194676 & 163332 & 1244164 & 1366212 & 1255006 & 1040802 & 993744 \\
\hline & $21.93 \%$ & $6.31 \%$ & $15.33 \%$ & $17.61 \%$ & $18.20 \%$ & $17.57 \%$ & $17.90 \%$ \\
\hline
\end{tabular}

THESSALONIKI

${ }^{*}$ Number of passenger vehicles ${ }^{* *}$ Number of heavy vehicles ${ }^{* * *}$ Percentage of heavy vehicles

\subsection{Traffic emission estimation using COPERT}

In order to calculate the air pollution produced by traffic along the road segment under study, the Computer Programme to Calculate Emissions from Road Transport (COPERT), and specifically its fourth version, COPERT4, was used. The development of COPERT is coordinated by the European Environment Agency, within the activities of the
European Topic Centre on Air Pollution and Climate Change Mitigation and supported scientifically and technically by Emisia S.A. and the Aristotle University of Thessaloniki Laboratory of Applied Thermodynamics. The basic operation of COPERT is the calculation of emissions of all major air pollutants during transport; Vehicles are classified into five main groups: passenger, light, heavy, mopeds and motorcycles. Then each group is divided into 
subcategories, depending on the engine type of the vehicle. This provides the user with a clear picture of the traffic composition, while the program's calculations are more accurate in view of the specific emissions of each engine. The pollutants that are calculated by COPERT are divided in turn into four categories. The first category includes basic pollutants for which exists a specific calculation methodology with specific emission indicators. The second category includes the pollutants which are calculated based on fuel consumption. The third category pollutants are calculated with a simplified methodology, given the lack of detailed data for their analytical calculation. Lastly, the fourth category includes pollutants that are estimated as a fraction of non-methane volatile

Table 6. Final input parameters for COPERT4 software calculations organic compound (NMVOC) emissions (Gkatzoflias et al., 2012).

The import in the software of the occurrence of each engine type, along with all the features that affect the pollutants production is an essential precondition for the correct calculation of pollutants. Data collected from the toll stations along the selected highway segment are transformed according to the records of the national fleet in circulation, for each specific year, that have been prepared for COPERT in order to be imported into the program. Table 6 presents the final input parameters for the required COPERT4 software calculations.

\begin{tabular}{|c|c|c|c|c|}
\hline \multirow[b]{2}{*}{ Segment } & \multirow[b]{2}{*}{ Axis Distance $(\mathrm{km})$} & \multicolumn{2}{|c|}{ Traffic Speed $\left(\mathrm{km} \mathrm{h}^{-1}\right)$} & \multirow[b]{2}{*}{ Traffic Volume (veh) } \\
\hline & & $\begin{array}{l}\text { Passenger } \\
\text { Vehicles }\end{array}$ & Heavy Vehicles & \\
\hline 1 & 36.2 & \multirow{9}{*}{130} & \multirow{9}{*}{85} & \multirow{9}{*}{$\begin{array}{c}\text { Annual traffic volumes and } \\
\text { passenger/heavy vehicle fleet shares } \\
\text { according to Table } 5\end{array}$} \\
\hline 2 & 80.3 & & & \\
\hline 3 & 47.2 & & & \\
\hline 4 & 130.1 & & & \\
\hline 5 & 83.4 & & & \\
\hline 6 & 41.8 & & & \\
\hline 7 & 31.5 & & & \\
\hline 8 & 18.4 & & & \\
\hline 9 & 26.1 & & & \\
\hline
\end{tabular}

It should be noted that in this analysis data were imported for two categories of vehicles, passenger cars and heavy vehicles, and the zero value was used for any other vehicle category, for which no data were available. The estimate of the frequency of each engine type in the road section under study was estimated using the data counted at the toll stations in conjunction with the national fleet data.

As for the traffic speeds that the program estimates, the higher speed limits were used, as specified by the highway road traffic regulations for the relevant vehicle categories i.e. $130 \mathrm{~km} \mathrm{~h}^{-1}$ for passenger vehicles and $85 \mathrm{~km} \mathrm{~h}^{-1}$ for heavy vehicles.

The pollutants that were chosen for the analysis were carbon dioxide $\left(\mathrm{CO}_{2}\right)$, nitrous oxides $\left(\mathrm{NO}_{\mathrm{x}}\right)$, particulate Table 7. Overall evolution of emissions per year

\begin{tabular}{cccccc}
\hline Year & NMVOC $(\mathbf{t})$ & $\mathbf{N O}_{\mathbf{x}}(\mathbf{t})$ & $\mathbf{N H}_{\mathbf{3}}(\mathbf{t})$ & $\mathbf{P M}_{\mathbf{2 . 5}}(\mathbf{t})$ & $\mathbf{\mathbf { C O } _ { \mathbf { 2 } } ( \mathbf { t } )}$ \\
\hline 2008 & 110489.251 & 6862.998 & 171.811 & 143.539 & 1137152.184 \\
\hline 2009 & 110040.745 & 5300.679 & 152.716 & 111.613 & 954944.846 \\
\hline 2010 & 87933.739 & 5260.055 & 156.098 & 118.214 & 984496.713 \\
\hline 2011 & 75078.305 & 4700.718 & 141.253 & 110.919 & 908337.959 \\
\hline 2012 & 59581.781 & 3764.949 & 119.343 & 91.768 & 767947.522 \\
\hline 2013 & 52589.470 & 3271.597 & 110.642 & 82.124 & 709149.478 \\
\hline 2014 & 44941.683 & 2753.952 & 98.930 & 71.827 & 637935.501 \\
\hline
\end{tabular}

matter ( $\left.\mathrm{PM}_{2.5}\right)$, ammonia $\left(\mathrm{NH}_{3}\right)$ and NMVOC, as they were considered as important indicators of pollutant emissions.

\section{Results}

Using traffic volume data from the toll stations, the COPERT4 data import followed, according to the process described above, for each toll station, for both directions (to Athens and to Thessaloniki), for a seven-year period (2008-2014). Thus, all the necessary analyses were produced emissions along Highway A1 are presented below.

Table 7 and Figures 4-8 present the general trend of traffic emissions per year during the period of 2008-2014. performed using the program and the results obtained for 


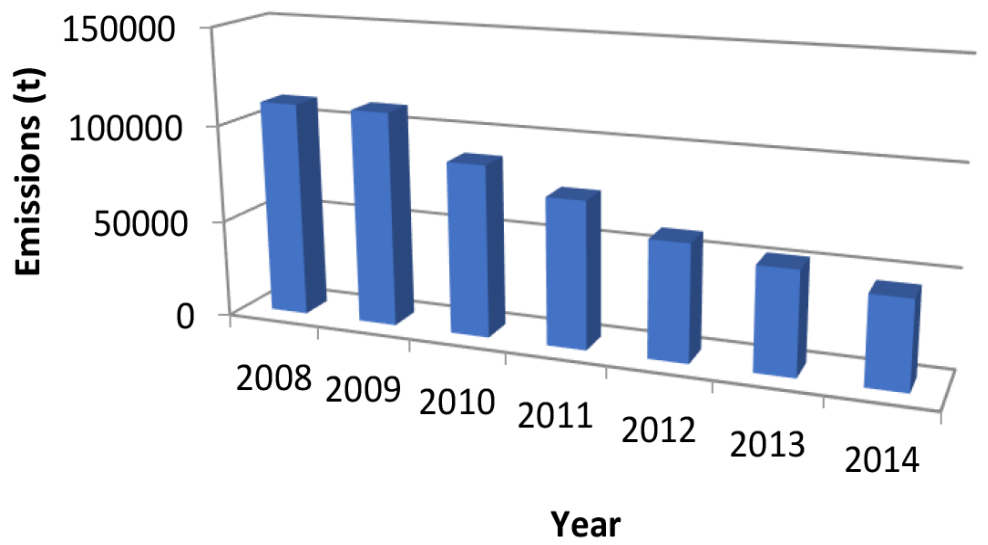

Figure 4. Evolution of total NMVOC emissions

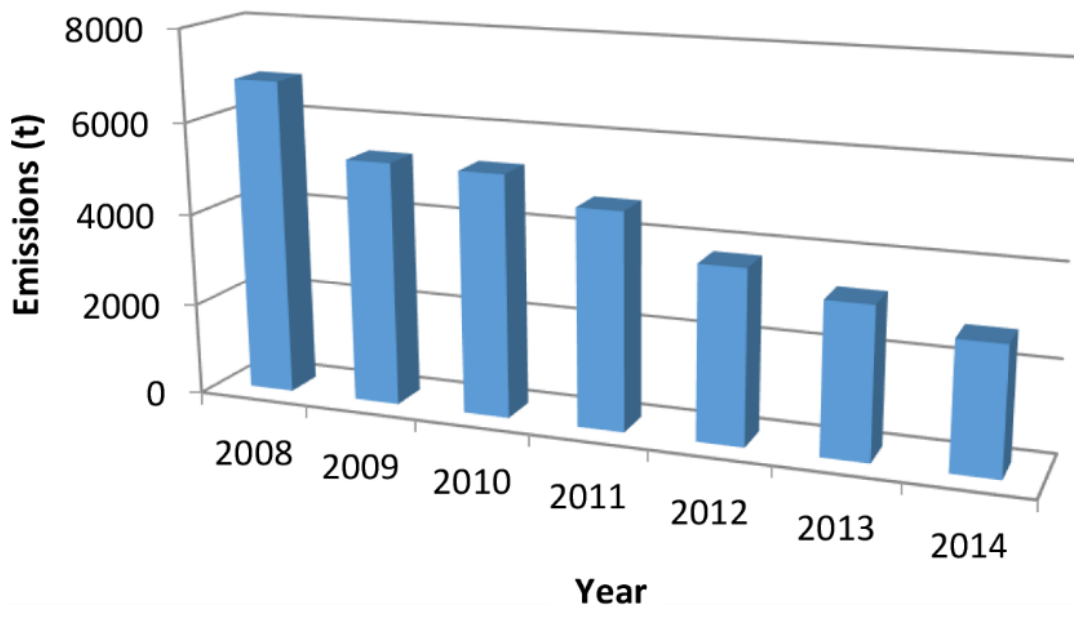

Figure 5. Evolution of total $\mathrm{NO}_{\mathrm{x}}$ emissions

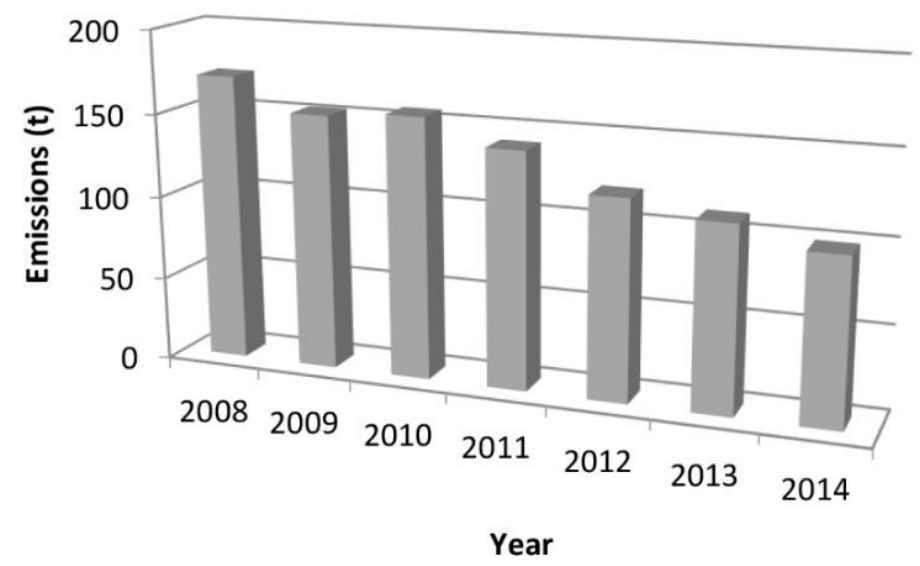

Figure 6. Evolution of total $\mathrm{NH}_{3}$ emissions 


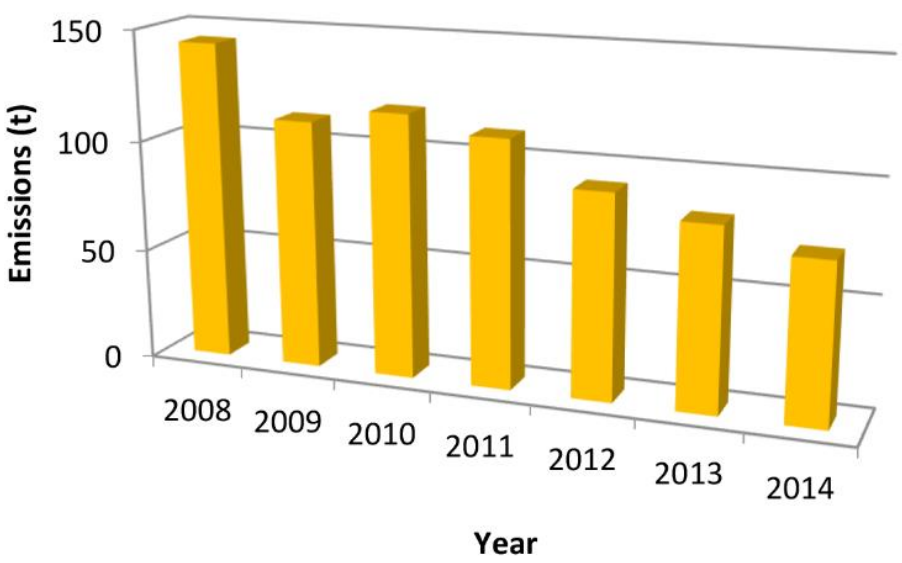

Figure 7. Evolution of total $\mathrm{PM}_{2.5}$ emissions

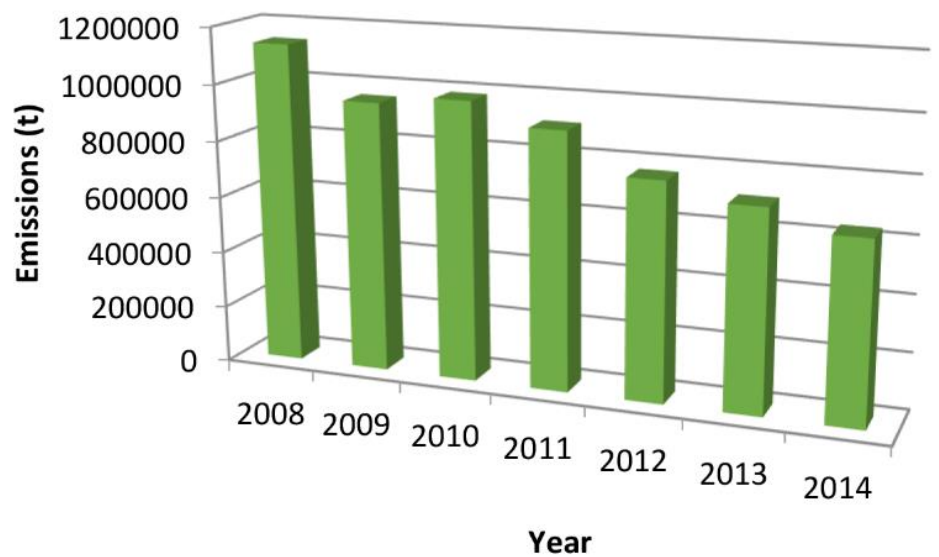

Figure 8. Evolution of total $\mathrm{CO}_{2}$ emissions

It can be observed that there is a downward trend in pollutant emissions over the years, with the largest decline in $\mathrm{NO}_{x}(59.87 \%)$ and the smallest in $\mathrm{NH}_{3}(42.42 \%)$. The total pollutant reductions between 2008 and 2014 are presented in Table 8. As expected, this decline in emissions is largely due to the overall reduction of the vehicle load.

Table 8. Decrease of all pollutants from 2008 to 2014

\begin{tabular}{ccccc}
\hline \multicolumn{5}{c}{ Overall decrease of all pollutants (2008 - 2014) } \\
\hline NMVOC & NOx $_{x}$ & $\mathbf{N H}_{3}$ & $\mathbf{P M}_{2.5}$ & $\mathbf{C O}_{2}$ \\
\hline $59.32 \%$ & $59.87 \%$ & $42.42 \%$ & $49.96 \%$ & $43.90 \%$ \\
\hline
\end{tabular}

\section{Discussion}

For the function of the COPERT program, the various vehicles are classified into five main groups (passenger, light, heavy, mopeds and motorcycles). Then each group is divided into subcategories, depending on the fuel, size and specifications of the engine, according to the European standards. This provides the user a clearer picture of the traffic composition at the national level, while the program's calculations are more accurate in view of the specific emissions of each engine type. In this study, traffic volume data are used only for two vehicle categories, passenger and heavy.
Comparing the vehicle fleet data for the years 2008 and 2014 some important conclusions are drawn. First, a significant drop in vehicles is observed between 2008 and 2014. There is a decrease of $44.36 \%$ for passenger and $30.66 \%$ for heavy vehicles. However, changes observed in vehicle categories that are in circulation are also notable. Generally, the passenger car fleet in Greece is characterized by dominance of gasoline to diesel in terms of fuel consumption. However, a trend towards larger displacement vehicles, better technology and better quality is noticed. Indeed, the fleet renewal with vehicles following improved technological standards, is performed faster in the larger displacement category irrespectively to fuel type, a fact that is directly connected with the fastest growth rate of medium and large vehicles. Similar conclusions can be drawn for heavy vehicles. For this category, there is also a turn in 2014 to classes of better and contemporary standards, which either did not exist in 2008 or their numbers increased significantly. The same applies to the two broad categories of heavy vehicles, articulated and non-articulated (rigid). 


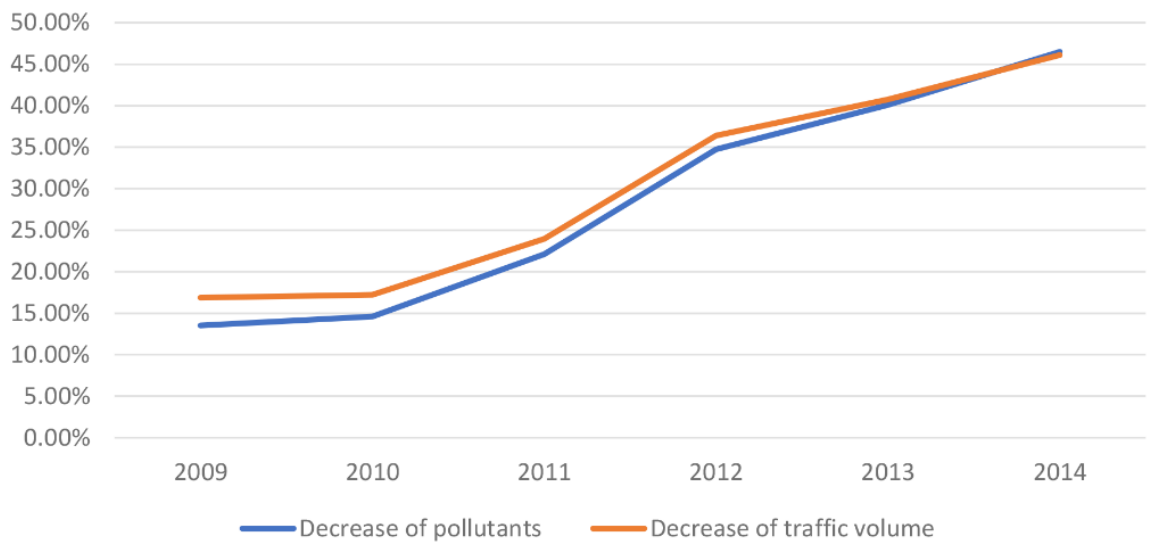

Figure 9. Relative decrease of pollutants and traffic volume compared to the levels of 2008

Finally, it is observed that, apart from the reduction of traffic volume, the size and engine technology, fuel type and vehicle age, play a key role in the reduction of pollutants in the studied road section, while an emerging trend towards vehicles larger displacement, better technology and better standards prevails (Figure 9).

\section{Conclusions}

This paper focuses on the diachronic evolution of exhaust emissions from traffic volume (passenger and heavy vehicles) along the main highway in Greece (Highway A1), connecting two largest urban areas of the country, Athens and Thessaloniki, during the economic recession period between 2008 and 2014. Based on the results of the analysis, a significant downward trend in the amount of different pollutant emissions was observed. The decline of emissions was between $42.42-59.87 \%$ and this is largely due to the reduction of traffic volume, but also due to a trend which generally prevails in Europe, for larger displacement vehicles with better technological standards.

\section{References}

Christoforou, Z., and Karlaftis, M. (2011), Urban restructuring and transportation in the financial crisis era: A study of the Athens CBD. In: European Transport Conference 2011, 1012/10/2011, Glasgow, UK, Association for European Transport, London.

Efthymiou, D., and Antoniou, C. (2017), Understanding the effects of economic crisis on public transport users' satisfaction and demand, Transport Policy, 53, 89-97. https://doi.org/10.1016/j.tranpol.2016.09.007

Enoch, M., Warren, J.P., Rios, H.V., and Menoyo, E.H. (2004), The effect of economic restrictions on transport practices in Cuba, Transport Policy, 11(1), 67-76. https://doi.org/10.1016/S0967-070X(03)00054-4

European Automobile Manufacturers Association. (2016), Statistics. Retrieved March 23, 2017, from http://www.acea.be/statistics

European Commission. (2015), EU Transport in Figures Statistical Pocketbook 2015, Publications Office of the European Union, Luxembourg. https://doi.org/10.2832/90922
European Environment Agency. (2015), EEA Report No 7/2015 Evaluating 15 years of transport and environmental policy integration - TERM 2015: Transport indicators tracking progress towards environmental targets in Europe, Luxembourg. https://doi.org/10.2800/214970

European Environment Agency. (2016), European annual statistics. Retrieved March 23, 2017, from http://www.eea.europa.eu/data-andmaps/data/aqereporting-1/estatistics

Gkatzoflias, D., Kouridis, C., Ntziachristos, L., and Samaras, Z. (2012), COPERT 4 Computer programme to calculate emissions from road transport User Manual, 9th edition, EMISIA S.A., Thessaloniki.

Hellenic Statistical Authority. (2017a), Cars and motorcycles, new and used, released for the first time in Greece (January 1985 - December 2016). Retrieved March 23, 2017, from http://www.statistics.gr/en/statistics/-/publication/SME18/

Hellenic Statistical Authority. (2017b), Gross Domestic Product (Flash Estimates) (1995 - 2016). Retrieved June 13, 2017, from http://www.statistics.gr/en/statistics/-/publication/SEL15/

Lee, S. (2010), Transport and the Recession: An Opportunity to Promote Sustainable Transport. International Planning Studies, 15(3), 213-226. https://doi.org/10.1080/13563475.2010.509475

Lyamani, H., Olmo, F.J., Foyo, I., and Alados-Arboledas, L. (2011), Black carbon aerosols over an urban area in south-eastern Spain: Changes detected after the 2008 economic crisis, Atmospheric Environment, 45(35), 6423-6432. https://doi.org/10.1016/j.atmosenv.2011.07.063

Nielsen, T.A.S. (2015), Changes in transport behavior during the financial crisis. An analysis of urban form, location and transport behavior in the greater Copenhagen area 20062011, Research in Transportation Economics, 51, 10-19. https://doi.org/10.1016/j.retrec.2015.07.003

Papagiannakis, A., Baraklianos, I., and Spyridonidou, A. (2016), Urban travel behaviour and household income in times of economic crisis: Challenges and perspectives for sustainable mobility, Transport Policy. https://doi.org/10.1016/j.tranpol.2016.12.006

Sobrino, N., and Monzon, A. (2014), The impact of the economic crisis and policy actions on GHG emissions from road 
transport in Spain, Energy Policy, 74, 486-498. https://doi.org/10.1016/j.enpol.2014.07.020

Wang, Z. (2009), Strengthening Investment in Transport Infrastructure to Address the Financial Crisis by Joint Effort,
In: 6th APEC Transportation Ministerial Meeting (TPTMM6) 27-29 April 2009, Manila, Philippines, Asia-Pacific Economic Cooperation, Manila. 
Annex - COPERT4 Input Data

\section{Afidnes Station}

\begin{tabular}{|c|c|c|c|c|c|c|c|c|c|}
\hline Sector & Subsector & Technology & 2008 & 2009 & 2010 & 2011 & 2012 & 2013 & 2014 \\
\hline Passenger Cars & Gasoline $0,8-1,4 \mathrm{I}$ & ECE $15 / 02$ & 36269 & 16717 & 6708 & 0 & 0 & 0 & 0 \\
\hline Passenger Cars & Gasoline $0,8-1,4$ I & ECE $15 / 03$ & 335102 & 252467 & 221392 & 160730 & 110490 & 83403 & 60162 \\
\hline Passenger Cars & Gasoline $0,8-1,4$ I & ECE 15/04 & 2070862 & 1690821 & 1588848 & 1216108 & 886023 & 690639 & 502048 \\
\hline Passenger Cars & Gasoline $0,8-1,4$ I & PC Euro 1 - 91/441/EEC & 2277714 & 1959888 & 1946425 & 1577761 & 1218697 & 1007051 & 774978 \\
\hline Passenger Cars & Gasoline $0,8-1,4$ I & PC Euro 2 - 94/12/EEC & 3681676 & 3270870 & 3371897 & 2853757 & 2316119 & 2024756 & 1660535 \\
\hline Passenger Cars & Gasoline $0,8-1,4$ I & PC Euro 3 - 98/69/EC Stage2000 & 3856203 & 3684751 & 3863964 & 3339079 & 2778889 & 2502982 & 2126236 \\
\hline Passenger Cars & Gasoline $0,8-1,4$ I & PC Euro 4 - 98/69/EC Stage2005 & 754945 & 867803 & 1330276 & 1427373 & 1426413 & 1507969 & 1479539 \\
\hline Passenger Cars & Gasoline $0,8-1,4$ I & PC Euro 5 - EC 715/2007 & 0 & 0 & 379953 & 600688 & 753958 & 936830 & 1043777 \\
\hline Passenger Cars & Gasoline 1,4-2,0। & ECE $15 / 02$ & 5234 & 2149 & 795 & 0 & 0 & 0 & 0 \\
\hline Passenger Cars & Gasoline 1,4-2,0। & ECE $15 / 03$ & 75810 & 54757 & 47887 & 34938 & 24595 & 19024 & 14001 \\
\hline Passenger Cars & Gasoline $1,4-2,0$ I & ECE $15 / 04$ & 541914 & 420322 & 386437 & 288909 & 205282 & 155819 & 110137 \\
\hline Passenger Cars & Gasoline 1,4-2,0। & PC Euro 1 - 91/441/EEC & 970480 & 807864 & 800339 & 646741 & 497669 & 409390 & 313397 \\
\hline Passenger Cars & Gasoline 1,4-2,0 I & PC Euro 2 - 94/12/EEC & 1505762 & 1292925 & 1327930 & 1118596 & 902540 & 783330 & 636835 \\
\hline Passenger Cars & Gasoline 1,4 - 2,0। & PC Euro 3 - 98/69/EC Stage2000 & 2487079 & 2334209 & 2445633 & 2110821 & 1753713 & 1575981 & 1334734 \\
\hline Passenger Cars & Gasoline 1,4-2,0I & PC Euro 4 - 98/69/EC Stage2005 & 565558 & 639468 & 843928 & 840929 & 798153 & 813185 & 776618 \\
\hline Passenger Cars & Gasoline 1,4-2,0 I & PC Euro 5 - EC 715/2007 & 0 & 0 & 155505 & 242323 & 304761 & 381586 & 429558 \\
\hline Passenger Cars & Gasoline $>2,0$ I & ECE $15 / 02$ & 2565 & 1396 & 742 & 0 & 0 & 0 & 0 \\
\hline Passenger Cars & Gasoline $>2,0 \mathrm{I}$ & ECE 15/03 & 4904 & 3811 & 3850 & 3210 & 2029 & 1237 & 536 \\
\hline Passenger Cars & Gasoline $>2,0$ I & ECE $15 / 04$ & 9707 & 7597 & 7730 & 6491 & 5257 & 4623 & 3846 \\
\hline Passenger Cars & Gasoline $>2,0 \mathrm{I}$ & PC Euro 1 - 91/441/EEC & 27635 & 21729 & 22212 & 18739 & 15249 & 13465 & 11252 \\
\hline Passenger Cars & Gasoline $>2,0 \mathrm{I}$ & PC Euro 2 - 94/12/EEC & 40128 & 31776 & 32714 & 27780 & 22757 & 20224 & 17003 \\
\hline Passenger Cars & Gasoline $>2,0 \mathrm{O}$ & PC Euro 3 - 98/69/EC Stage2000 & 171090 & 154630 & 160560 & 137468 & 113494 & 101621 & 86062 \\
\hline Passenger Cars & Gasoline $>2,0 \mathrm{I}$ & PC Euro 4 - 98/69/EC Stage2005 & 55897 & 60554 & 70293 & 64490 & 56887 & 54264 & 48825 \\
\hline Passenger Cars & Gasoline $>2,0 \mathrm{I}$ & PC Euro 5 - EC 715/2007 & 0 & 0 & 6547 & 9708 & 11821 & 14394 & 15786 \\
\hline Passenger Cars & Diesel $1,4-2,0$ I & Conventional & 7163 & 4154 & 2350 & 1024 & 400 & 155 & 52 \\
\hline Passenger Cars & Diesel 1,4-2,0I & PC Euro 1 - 91/441/EEC & 41470 & 32196 & 24984 & 15314 & 8645 & 5038 & 2638 \\
\hline Passenger Cars & Diesel $1,4-2,0$ I & PC Euro $2-94 / 12 /$ EEC & 37077 & 29245 & 23041 & 14316 & 8179 & 4813 & 2538 \\
\hline Passenger Cars & Diesel 1,4-2,0। & PC Euro 3 - 98/69/EC Stage2000 & 108714 & 105681 & 100952 & 77736 & 56276 & 42965 & 30097 \\
\hline Passenger Cars & Diesel $1,4-2,0$ I & PC Euro 4 - 98/69/EC Stage2005 & 16183 & 19630 & 38903 & 46252 & 47727 & 50194 & 47897 \\
\hline Passenger Cars & Diesel $1,4-2,0 \mid$ & PC Euro 5 - EC 715/2007 & 0 & 0 & 17239 & 28202 & 34406 & 40693 & 42727 \\
\hline Passenger Cars & Diesel $>2,0$ । & Conventional & 804 & 322 & 119 & 29 & 5 & 0 & 0 \\
\hline Passenger Cars & Diesel $>2,0 \mathrm{I}$ & PC Euro 1 - 91/441/EEC & 10889 & 7685 & 5682 & 3194 & 1585 & 774 & 315 \\
\hline Passenger Cars & Diesel $>2,0$ I & PC Euro 2 - 94/12/EEC & 9076 & 6579 & 4966 & 2820 & 1399 & 679 & 273 \\
\hline Passenger Cars & Diesel $>2,0$ I & PC Euro 3 - 98/69/EC Stage2000 & 40329 & 38722 & 39120 & 31926 & 24439 & 19570 & 14190 \\
\hline Passenger Cars & Diesel $>2,0$ I & PC Euro 4 - 98/69/EC Stage2005 & 7818 & 9163 & 13994 & 15233 & 15178 & 15837 & 15222 \\
\hline Passenger Cars & Diesel $>2,0$ I & PC Euro 5 - EC 715/2007 & 0 & 0 & 4014 & 6634 & 8321 & 10217 & 11215 \\
\hline Passenger Cars & LPG & Conventional & 8 & 0 & 0 & 0 & 0 & 0 & 0 \\
\hline Passenger Cars & LPG & PC Euro 1 - 91/441/EEC & 137 & 32 & 7 & 0 & 0 & 0 & 0 \\
\hline Passenger Cars & LPG & PC Euro 2 - 94/12/EEC & 2303 & 1414 & 836 & 332 & 96 & 20 & 2 \\
\hline Passenger Cars & LPG & PC Euro 3 - 98/69/EC Stage2000 & 9209 & 8664 & 8446 & 6537 & 4658 & 3426 & 2271 \\
\hline Passenger Cars & LPG & PC Euro 4 - 98/69/EC Stage2005 & 1712 & 2008 & 3033 & 3307 & 3305 & 3441 & 3274 \\
\hline Passenger Cars & LPG & PC Euro 5 - EC 715/2007 & 0 & 0 & 851 & 1431 & 1821 & 2244 & 2447 \\
\hline Passenger Cars & 2-Stroke & Conventional & 0 & 0 & 167775 & 217060 & 242376 & 276184 & 286482 \\
\hline Passenger Cars & Hybrid Gasoline 1,4 - 2,0I & PC Euro 4 - 98/69/EC Stage2005 & 0 & 0 & 191510 & 248391 & 277713 & 316694 & 328671 \\
\hline Heavy Duty Trucks & Rigid $<=7,5 \mathrm{t}$ & Conventional & 243154 & 221768 & 247347 & 202973 & 167574 & 146968 & 115813 \\
\hline Heavy Duty Trucks & Rigid $<=7,5 \mathrm{t}$ & HD Euro I - 91/542/EEC Stage I & 107791 & 98407 & 109975 & 91491 & 77835 & 70412 & 57910 \\
\hline Heavy Duty Trucks & Rigid $<=7,5 \mathrm{t}$ & HD Euro II - 91/542/EEC Stage II & 176990 & 161577 & 180559 & 150218 & 127797 & 115607 & 95086 \\
\hline Heavy Duty Trucks & Rigid $<=7,5 \mathrm{t}$ & HD Euro III - 2000 Standards & 201161 & 183639 & 205226 & 170732 & 145251 & 131400 & 108073 \\
\hline Heavy Duty Trucks & Rigid $<=7,5 \mathrm{t}$ & HD Euro IV - 2005 Standards & 82358 & 102321 & 116267 & 116218 & 116620 & 121356 & 99812 \\
\hline Heavy Duty Trucks & Rigid $<=7,5 \mathrm{t}$ & HD Euro V - 2008 Standards & 0 & 23681 & 28218 & 42978 & 55997 & 69650 & 70673 \\
\hline Heavy Duty Trucks & Rigid $<=7,5 \mathrm{t}$ & HD Euro VI & 0 & 0 & 0 & 0 & 0 & 0 & 17574 \\
\hline Heavy Duty Trucks & Rigid $7,5-12 \mathrm{t}$ & Conventional & 109390 & 99766 & 111274 & 91320 & 75385 & 66124 & 52104 \\
\hline Heavy Duty Trucks & Rigid 7,5 - $12 \mathrm{t}$ & HD Euro I - 91/542/EEC Stage I & 48496 & 44269 & 49478 & 41161 & 35017 & 31679 & 26052 \\
\hline Heavy Duty Trucks & Rigid 7,5 - $12 \mathrm{t}$ & HD Euro II - 91/542/EEC Stage II & 79622 & 72692 & 81236 & 67581 & 57497 & 52009 & 42778 \\
\hline Heavy Duty Trucks & Rigid $7,5-12 \mathrm{t}$ & HD Euro III - 2000 Standards & 90499 & 82620 & 92332 & 76816 & 65349 & 59117 & 48622 \\
\hline Heavy Duty Trucks & Rigid 7,5 - $12 \mathrm{t}$ & HD Euro IV - 2005 Standards & 37051 & 46033 & 52308 & 52289 & 52471 & 54595 & 44904 \\
\hline Heavy Duty Trucks & Rigid $7,5-12 \mathrm{t}$ & HD Euro V - 2008 Standards & 0 & 10660 & 12694 & 19335 & 25189 & 31337 & 31798 \\
\hline Heavy Duty Trucks & Rigid $7,5-12 \mathrm{t}$ & HD Euro VI & 0 & 0 & 0 & 0 & 0 & 0 & 7905 \\
\hline Heavy Duty Trucks & Rigid $12-14 \mathrm{t}$ & Conventional & 30606 & 27913 & 31136 & 25545 & 21088 & 18496 & 14573 \\
\hline Heavy Duty Trucks & Rigid $12-14 \mathrm{t}$ & HD Euro I - 91/542/EEC Stage I & 13564 & 12385 & 13837 & 11517 & 9794 & 8864 & 7286 \\
\hline Heavy Duty Trucks & Rigid $12-14 t$ & HD Euro II - 91/542/EEC Stage II & 22273 & 20337 & 22725 & 18907 & 16087 & 14550 & 11966 \\
\hline Heavy Duty Trucks & Rigid $12-14 \mathrm{t}$ & HD Euro III - 2000 Standards & 25317 & 23113 & 25832 & 21484 & 18279 & 16539 & 13599 \\
\hline Heavy Duty Trucks & Rigid $12-14 \mathrm{t}$ & HD Euro IV - 2005 Standards & 10366 & 12877 & 14636 & 14627 & 14679 & 15272 & 12565 \\
\hline Heavy Duty Trucks & Rigid $12-14 t$ & HD Euro V - 2008 Standards & 0 & 2978 & 3551 & 5411 & 7052 & 8770 & 8893 \\
\hline Heavy Duty Trucks & Rigid $12-14 \mathrm{t}$ & HD Euro VI & 0 & 0 & 0 & 0 & 0 & 0 & 2212 \\
\hline Heavy Duty Trucks & Rigid $14-20 t$ & Conventional & 84295 & 76885 & 85752 & 70368 & 58097 & 50952 & 40151 \\
\hline Heavy Duty Trucks & Rigid $14-20 t$ & HD Euro I - 91/542/EEC Stage I & 37369 & 34120 & 38127 & 31717 & 26981 & 24408 & 20076 \\
\hline Heavy Duty Trucks & Rigid $14-20 t$ & HD Euro II - 91/542/EEC Stage II & 61357 & 56018 & 62594 & 52079 & 44302 & 40084 & 32964 \\
\hline Heavy Duty Trucks & Rigid $14-20 t$ & HD Euro III - 2000 Standards & 69738 & 63671 & 71149 & 59193 & 50354 & 45553 & 37466 \\
\hline Heavy Duty Trucks & Rigid $14-20 \mathrm{t}$ & HD Euro IV - 2005 Standards & 28554 & 35469 & 40302 & 40286 & 40435 & 42073 & 34602 \\
\hline Heavy Duty Trucks & Rigid $14-20 \mathrm{t}$ & HD Euro V - 2008 Standards & 0 & 8212 & 9787 & 14903 & 19413 & 24152 & 24505 \\
\hline Heavy Duty Trucks & Rigid $14-20 t$ & HD Euro VI & 0 & 0 & 0 & 0 & 0 & 0 & 6095 \\
\hline Heavy Duty Trucks & Rigid $20-26 \mathrm{t}$ & Conventional & 68515 & 62485 & 69696 & 57196 & 47220 & 41413 & 32634 \\
\hline Heavy Duty Trucks & Rigid $20-26 \mathrm{t}$ & HD Euro I - 91/542/EEC Stage I & 30375 & 27729 & 30981 & 25783 & 21930 & 19840 & 16317 \\
\hline Heavy Duty Trucks & Rigid $20-26 \mathrm{t}$ & HD Euro II - 91/542/EEC Stage II & 49864 & 45522 & 50876 & 42331 & 36009 & 32572 & 26789 \\
\hline Heavy Duty Trucks & Rigid $20-26 \mathrm{t}$ & HD Euro III - 2000 Standards & 56675 & 51748 & 57823 & 48104 & 40926 & 37024 & 30449 \\
\hline Heavy Duty Trucks & Rigid $20-26 \mathrm{t}$ & HD Euro IV - 2005 Standards & 23208 & 28828 & 32756 & 32745 & 32858 & 34196 & 28126 \\
\hline Heavy Duty Trucks & Rigid $20-26 \mathrm{t}$ & HD Euro V - 2008 Standards & 0 & 6670 & 7956 & 12107 & 15779 & 19623 & 19911 \\
\hline Heavy Duty Trucks & Rigid $20-26 \mathrm{t}$ & HD Euro VI & 0 & 0 & 0 & 0 & 0 & 0 & 4950 \\
\hline Heavy Duty Trucks & Rigid $26-28 \mathrm{t}$ & Conventional & 173 & 164 & 178 & 152 & 125 & 109 & 86 \\
\hline Heavy Duty Trucks & Rigid $26-28 \mathrm{t}$ & HD Euro I - 91/542/EEC Stage I & 77 & 67 & 78 & 67 & 58 & 54 & 39 \\
\hline Heavy Duty Trucks & Rigid $26-28 \mathrm{t}$ & HD Euro II - 91/542/EEC Stage II & 125 & 116 & 133 & 114 & 92 & 85 & 72 \\
\hline Heavy Duty Trucks & Rigid $26-28 \mathrm{t}$ & HD Euro III - 2000 Standards & 145 & 135 & 144 & 124 & 108 & 93 & 79 \\
\hline Heavy Duty Trucks & Rigid $26-28 \mathrm{t}$ & HD Euro IV - 2005 Standards & 58 & 77 & 89 & 86 & 83 & 85 & 72 \\
\hline Heavy Duty Trucks & Rigid $26-28 \mathrm{t}$ & HD Euro V - 2008 Standards & 0 & 19 & 22 & 29 & 42 & 54 & 53 \\
\hline Heavy Duty Trucks & Rigid $26-28 \mathrm{t}$ & HD Euro VI & 0 & 0 & 0 & 0 & 0 & 0 & 13 \\
\hline Heavy Duty Trucks & Rigid $28-32 \mathrm{t}$ & Conventional & 6416 & 5850 & 6525 & 5354 & 4418 & 3876 & 3054 \\
\hline Heavy Duty Trucks & Rigid $28-32 \mathrm{t}$ & HD Euro I - 91/542/EEC Stage I & 2842 & 2593 & 2896 & 2416 & 2050 & 1857 & 1527 \\
\hline
\end{tabular}




\begin{tabular}{|c|c|c|c|c|c|c|c|c|c|}
\hline Sector & Subsector & Technology & 2008 & 2009 & 2010 & 2011 & 2012 & 2013 & 2014 \\
\hline Heavy Duty Trucks & Rigid $28-32 \mathrm{t}$ & HD Euro II - 91/542/EEC Stage II & 4672 & 4260 & 4760 & 3966 & 3376 & 3053 & 2508 \\
\hline Heavy Duty Trucks & Rigid $28-32 \mathrm{t}$ & HD Euro III - 2000 Standards & 5308 & 4848 & 5415 & 4508 & 3834 & 3465 & 2850 \\
\hline Heavy Duty Trucks & Rigid $28-32 \mathrm{t}$ & HD Euro IV - 2005 Standards & 2177 & 2699 & 3063 & 3062 & 3076 & 3201 & 2633 \\
\hline Heavy Duty Trucks & Rigid $28-32 \mathrm{t}$ & HD Euro V - 2008 Standards & 0 & 626 & 743 & 1132 & 1475 & 1841 & 1863 \\
\hline Heavy Duty Trucks & Rigid $28-32 \mathrm{t}$ & HD Euro VI & 0 & 0 & 0 & 0 & 0 & 0 & 461 \\
\hline Heavy Duty Trucks & Rigid $>32 t$ & Conventional & 35385 & 32279 & 35996 & 29540 & 24389 & 21394 & 16857 \\
\hline Heavy Duty Trucks & Rigid $>32 t$ & HD Euro I - 91/542/EEC Stage I & 15693 & 14323 & 16001 & 13315 & 11328 & 10246 & 8432 \\
\hline Heavy Duty Trucks & Rigid $>32 t$ & HD Euro II - 91/542/EEC Stage II & 25760 & 23518 & 26276 & 21865 & 18596 & 16826 & 13842 \\
\hline Heavy Duty Trucks & Rigid $>32 t$ & HD Euro III - 2000 Standards & 29277 & 26727 & 29871 & 24851 & 21138 & 19125 & 15731 \\
\hline Heavy Duty Trucks & Rigid $>32 \mathrm{t}$ & HD Euro IV - 2005 Standards & 11984 & 14891 & 16922 & 16919 & 16971 & 17665 & 14527 \\
\hline Heavy Duty Trucks & Rigid $>32 t$ & HD Euro V - 2008 Standards & 0 & 3451 & 4106 & 6258 & 8152 & 10138 & 10288 \\
\hline Heavy Duty Trucks & Rigid $>32 \mathrm{t}$ & HD Euro VI & 0 & 0 & 0 & 0 & 0 & 0 & 2560 \\
\hline Heavy Duty Trucks & Articulated $14-20 t$ & Conventional & 14075 & 12713 & 14270 & 11898 & 9927 & 8809 & 7050 \\
\hline Heavy Duty Trucks & Articulated $14-20 \mathrm{t}$ & HD Euro I - 91/542/EEC Stage I & 2447 & 2217 & 2497 & 2083 & 1784 & 1616 & 1336 \\
\hline Heavy Duty Trucks & Articulated $14-20 \mathrm{t}$ & HD Euro II - 91/542/EEC Stage II & 3333 & 3036 & 3418 & 2863 & 2442 & 2222 & 1836 \\
\hline Heavy Duty Trucks & Articulated $14-20 t$ & HD Euro III - 2000 Standards & 5347 & 4896 & 5526 & 4641 & 3976 & 3628 & 3008 \\
\hline Heavy Duty Trucks & Articulated $14-20 \mathrm{t}$ & HD Euro IV - 2005 Standards & 3092 & 3846 & 4294 & 4175 & 4159 & 4311 & 3581 \\
\hline Heavy Duty Trucks & Articulated $14-20 \mathrm{t}$ & HD Euro V - 2008 Standards & 0 & 887 & 954 & 1370 & 1800 & 2261 & 2317 \\
\hline Heavy Duty Trucks & Articulated $14-20 \mathrm{t}$ & HD Euro VI & 0 & 0 & 0 & 0 & 0 & 0 & 573 \\
\hline Heavy Duty Trucks & Articulated $20-28 \mathrm{t}$ & Conventional & 9229 & 8328 & 9354 & 7799 & 6510 & 5772 & 4621 \\
\hline Heavy Duty Trucks & Articulated $20-28 \mathrm{t}$ & HD Euro I - 91/542/EEC Stage I & 1599 & 1455 & 1631 & 1370 & 1167 & 1056 & 875 \\
\hline Heavy Duty Trucks & Articulated $20-28 \mathrm{t}$ & HD Euro II - 91/542/EEC Stage II & 2187 & 1985 & 2241 & 1874 & 1600 & 1460 & 1205 \\
\hline Heavy Duty Trucks & Articulated $20-28 \mathrm{t}$ & HD Euro III - 2000 Standards & 3507 & 3210 & 3617 & 3043 & 2609 & 2377 & 1968 \\
\hline Heavy Duty Trucks & Articulated $20-28 \mathrm{t}$ & HD Euro IV - 2005 Standards & 2023 & 2525 & 2818 & 2730 & 2726 & 2828 & 2343 \\
\hline Heavy Duty Trucks & Articulated $20-28 \mathrm{t}$ & HD Euro V - 2008 Standards & 0 & 578 & 632 & 894 & 1184 & 1484 & 1520 \\
\hline Heavy Duty Trucks & Articulated $20-28 \mathrm{t}$ & HD Euro VI & 0 & 0 & 0 & 0 & 0 & 0 & 375 \\
\hline Heavy Duty Trucks & Articulated $28-34 \mathrm{t}$ & Conventional & 7283 & 6573 & 7379 & 6153 & 5135 & 4560 & 3647 \\
\hline Heavy Duty Trucks & Articulated $28-34 \mathrm{t}$ & HD Euro I - 91/542/EEC Stage I & 1262 & 1147 & 1287 & 1075 & 917 & 839 & 691 \\
\hline Heavy Duty Trucks & Articulated $28-34 \mathrm{t}$ & HD Euro II - 91/542/EEC Stage II & 1724 & 1571 & 1764 & 1474 & 1267 & 1150 & 948 \\
\hline Heavy Duty Trucks & Articulated $28-34 \mathrm{t}$ & HD Euro III - 2000 Standards & 2765 & 2535 & 2852 & 2397 & 2059 & 1880 & 1553 \\
\hline Heavy Duty Trucks & Articulated $28-34 \mathrm{t}$ & HD Euro IV - 2005 Standards & 1599 & 1985 & 2219 & 2159 & 2151 & 2229 & 1850 \\
\hline Heavy Duty Trucks & Articulated $28-34 \mathrm{t}$ & HD Euro V - 2008 Standards & 0 & 463 & 499 & 704 & 934 & 1173 & 1198 \\
\hline Heavy Duty Trucks & Articulated $28-34 \mathrm{t}$ & HD Euro VI & 0 & 0 & 0 & 0 & 0 & 0 & 296 \\
\hline Heavy Duty Trucks & Articulated $34-40 \mathrm{t}$ & Conventional & 110980 & 100229 & 112483 & 93783 & 78310 & 69487 & 55567 \\
\hline Heavy Duty Trucks & Articulated $34-40 \mathrm{t}$ & HD Euro I - 91/542/EEC Stage I & 19267 & 17513 & 19663 & 16434 & 14045 & 12756 & 10531 \\
\hline Heavy Duty Trucks & Articulated $34-40 \mathrm{t}$ & HD Euro II - 91/542/EEC Stage II & 26300 & 23942 & 26931 & 22540 & 19280 & 17533 & 14487 \\
\hline Heavy Duty Trucks & Articulated $34-40 t$ & HD Euro III - 2000 Standards & 42196 & 38582 & 43553 & 36577 & 31383 & 28618 & 23709 \\
\hline Heavy Duty Trucks & Articulated $34-40 \mathrm{t}$ & HD Euro IV - 2005 Standards & 24364 & 30332 & 33866 & 32897 & 32783 & 33986 & 28218 \\
\hline Heavy Duty Trucks & Articulated $34-40 \mathrm{t}$ & HD Euro V - 2008 Standards & 0 & 7007 & 7545 & 10775 & 14220 & 17844 & 18292 \\
\hline Heavy Duty Trucks & Articulated $34-40 \mathrm{t}$ & HD Euro VI & 0 & 0 & 0 & 0 & 0 & 0 & 4529 \\
\hline Heavy Duty Trucks & Articulated $40-50 \mathrm{t}$ & Conventional & 520 & 463 & 522 & 437 & 367 & 326 & 257 \\
\hline Heavy Duty Trucks & Articulated $40-50 \mathrm{t}$ & HD Euro I - 91/542/EEC Stage I & 87 & 77 & 89 & 76 & 67 & 62 & 46 \\
\hline Heavy Duty Trucks & Articulated $40-50 \mathrm{t}$ & HD Euro II - 91/542/EEC Stage II & 125 & 116 & 122 & 105 & 92 & 85 & 66 \\
\hline Heavy Duty Trucks & Articulated $40-50 \mathrm{t}$ & HD Euro III - 2000 Standards & 193 & 183 & 200 & 171 & 150 & 132 & 112 \\
\hline Heavy Duty Trucks & Articulated $40-50 \mathrm{t}$ & HD Euro IV - 2005 Standards & 116 & 145 & 155 & 152 & 150 & 155 & 132 \\
\hline Heavy Duty Trucks & Articulated $40-50 \mathrm{t}$ & HD Euro V - 2008 Standards & 0 & 29 & 33 & 48 & 67 & 85 & 86 \\
\hline Heavy Duty Trucks & Articulated $40-50 \mathrm{t}$ & HD Euro VI & 0 & 0 & 0 & 0 & 0 & 0 & 20 \\
\hline Heavy Duty Trucks & Articulated $50-60 \mathrm{t}$ & Conventional & 212 & 193 & 222 & 181 & 150 & 132 & 105 \\
\hline Heavy Duty Trucks & Articulated $50-60 \mathrm{t}$ & HD Euro I - 91/542/EEC Stage I & 39 & 39 & 33 & 29 & 25 & 23 & 20 \\
\hline Heavy Duty Trucks & Articulated $50-60 \mathrm{t}$ & HD Euro II - 91/542/EEC Stage II & 48 & 48 & 55 & 48 & 33 & 31 & 26 \\
\hline Heavy Duty Trucks & Articulated $50-60 \mathrm{t}$ & HD Euro III - 2000 Standards & 77 & 77 & 89 & 67 & 58 & 54 & 46 \\
\hline Heavy Duty Trucks & Articulated $50-60 \mathrm{t}$ & HD Euro IV - 2005 Standards & 48 & 58 & 67 & 67 & 67 & 62 & 53 \\
\hline Heavy Duty Trucks & Articulated $50-60 \mathrm{t}$ & HD Euro V - 2008 Standards & 0 & 10 & 11 & 19 & 25 & 31 & 33 \\
\hline Heavy Duty Trucks & Articulated $50-60 \mathrm{t}$ & HD Euro VI & 0 & 0 & 0 & 0 & 0 & 0 & 7 \\
\hline
\end{tabular}




\section{Thiva Station}

\begin{tabular}{|c|c|c|c|c|c|c|}
\hline Sector & Subsector & Technology & 2008 & 2009 & 2010 & 2011 \\
\hline Passenger Cars & Gasoline 0,8 - 1,4 I & ECE 15/02 & 17919 & 4709 & 1915 & 0 \\
\hline Passenger Cars & Gasoline $0,8-1,4 \mathrm{I}$ & ECE 15/03 & 165563 & 71122 & 63209 & 46636 \\
\hline Passenger Cars & Gasoline $0,8-1,4$ I & ECE 15/04 & 1023146 & 476316 & 453626 & 352855 \\
\hline Passenger Cars & Gasoline $0,8-1,4$ I & PC Euro 1 - 91/441/EEC & 1125345 & 552115 & 555717 & 457788 \\
\hline Passenger Cars & Gasoline $0,8-1,4$ I & PC Euro 2 - 94/12/EEC & 1818997 & 921427 & 962698 & 828020 \\
\hline Passenger Cars & Gasoline $0,8-1,4$ I & $\begin{array}{l}\text { PC Euro } 3 \text { - 98/69/EC Stage2000 } \\
\end{array}$ & 1905225 & 1038021 & 1103187 & 968836 \\
\hline Passenger Cars & Gasoline $0,8-1,4 \mathrm{I}$ & $\begin{array}{l}\text { PC Euro } 4 \text { - 98/69/EC Stage2005 } \\
\end{array}$ & 372994 & 244466 & 379802 & 414153 \\
\hline Passenger Cars & Gasoline $0,8-1,4 \mathrm{I}$ & PC Euro 5 - EC 715/2007 & 0 & 0 & 108479 & 174290 \\
\hline Passenger Cars & Gasoline 1,4-2,0 I & ECE $15 / 02$ & 2586 & 605 & 227 & 0 \\
\hline Passenger Cars & Gasoline 1,4-2,0I & ECE 15/03 & 37455 & 15425 & 13672 & 10137 \\
\hline Passenger Cars & Gasoline $1,4-2,0$ I & ECE $15 / 04$ & 267742 & 118408 & 110330 & 83827 \\
\hline Passenger Cars & Gasoline 1,4-2,01 & PC Euro 1 - 91/441/EEC & 479483 & 227581 & 228502 & 187652 \\
\hline Passenger Cars & Gasoline 1,4-2,0 I & PC Euro 2 - 94/12/EEC & 743948 & 364226 & 379132 & 324561 \\
\hline Passenger Cars & Gasoline 1,4-2,0। & PC Euro 3 - 98/69/EC Stage2000 & 1228785 & 657563 & 698244 & 612456 \\
\hline Passenger Cars & Gasoline 1,4-2,0। & $\begin{array}{l}\text { PC Euro } 4 \text { - 98/69/EC Stage2005 } \\
\end{array}$ & 279424 & 180143 & 240947 & 243996 \\
\hline Passenger Cars & Gasoline $1,4-2,01$ & PC Euro 5 - EC 715/2007 & 0 & 0 & 44398 & 70310 \\
\hline Passenger Cars & Gasoline $>2,0$ I & ECE $15 / 02$ & 1267 & 393 & 212 & 0 \\
\hline Passenger Cars & Gasoline $>2,0 \mathrm{I}$ & ECE 15/03 & 2423 & 1073 & 1099 & 931 \\
\hline Passenger Cars & Gasoline $>2,0 \mathrm{I}$ & ECE 15/04 & 4796 & 2140 & 2207 & 1883 \\
\hline Passenger Cars & Gasoline $>2,0 \mathrm{I}$ & PC Euro 1 - 91/441/EEC & 13653 & 6121 & 6342 & 5437 \\
\hline Passenger Cars & Gasoline $>2,0 \mathrm{I}$ & PC Euro 2 - 94/12/EEC & 19826 & 8951 & 9340 & 8060 \\
\hline Passenger Cars & Gasoline $>2,0 \mathrm{I}$ & PC Euro 3 - 98/69/EC Stage2000 & 84530 & 43560 & 45841 & 39886 \\
\hline Passenger Cars & Gasoline $>2,0$ I & $\begin{array}{l}\text { PC Euro } 4 \text { - 98/69/EC Stage2005 } \\
\end{array}$ & 27617 & 17058 & 20069 & 18712 \\
\hline Passenger Cars & Gasoline $>2,0 \mathrm{I}$ & PC Euro 5 - EC 715/2007 & 0 & 0 & 1869 & 2817 \\
\hline Passenger Cars & Diesel 1,4-2,0 I & Conventional & 3539 & 1170 & 671 & 297 \\
\hline Passenger Cars & Diesel $1,4-2,0$ I & PC Euro 1 - 91/441/EEC & 20489 & 9070 & 7133 & 4443 \\
\hline Passenger Cars & Diesel $1,4-2,0$ I & PC Euro 2 - 94/12/EEC & 18318 & 8238 & 6578 & 4154 \\
\hline Passenger Cars & Diesel $1,4-2,0$ I & PC Euro 3 - 98/69/EC Stage2000 & 53712 & 29771 & 28822 & 22555 \\
\hline Passenger Cars & Diesel 1,4-2,0 I & $\begin{array}{l}\text { PC Euro } 4 \text { - 98/69/EC Stage2005 } \\
\end{array}$ & 7995 & 5530 & 11107 & 13420 \\
\hline Passenger Cars & Diesel $1,4-2,0$ I & PC Euro 5 - EC 715/2007 & 0 & 0 & 4922 & 8183 \\
\hline Passenger Cars & Diesel >2,0 I & Conventional & 397 & 91 & 34 & 8 \\
\hline Passenger Cars & Diesel $>2,0 \mathrm{I}$ & PC Euro 1 - 91/441/EEC & 5380 & 2165 & 1622 & 927 \\
\hline Passenger Cars & Diesel $>2,0$ I & PC Euro 2 - 94/12/EEC & 4484 & 1853 & 1418 & 818 \\
\hline Passenger Cars & Diesel $>2,0$ I & $\begin{array}{l}\text { PC Euro } 3 \text { - 98/69/EC Stage2000 } \\
\end{array}$ & 19925 & 10908 & 11169 & 9263 \\
\hline Passenger Cars & Diesel $>2,0 \mathrm{I}$ & $\begin{array}{l}\text { PC Euro } 4 \text { - 98/69/EC Stage2005 } \\
\end{array}$ & 3863 & 2581 & 3995 & 4420 \\
\hline Passenger Cars & Diesel >2,0 I & PC Euro 5 - EC $715 / 2007$ & 0 & 0 & 1146 & 1925 \\
\hline Passenger Cars & LPG & Conventional & 4 & 0 & 0 & 0 \\
\hline Passenger Cars & LPG & PC Euro 1 - 91/441/EEC & 68 & 9 & 2 & 0 \\
\hline Passenger Cars & LPG & PC Euro 2 - 94/12/EEC & 1138 & 398 & 239 & 96 \\
\hline Passenger Cars & LPG & $\begin{array}{l}\text { PC Euro } 3 \text { - 98/69/EC Stage2000 } \\
\end{array}$ & 4550 & 2441 & 2411 & 1897 \\
\hline Passenger Cars & LPG & PC Euro 4 - 98/69/EC Stage2005 & 846 & 566 & 866 & 960 \\
\hline Passenger Cars & LPG & PC Euro 5 - EC $715 / 2007$ & 0 & 0 & 243 & 415 \\
\hline Passenger Cars & 2-Stroke & Conventional & 0 & 0 & 47901 & 62980 \\
\hline Passenger Cars & Hybrid Gasoline $1,4-2,0$ I & $\begin{array}{l}\text { PC Euro } 4 \text { - 98/69/EC Stage2005 } \\
\end{array}$ & 0 & 0 & 54677 & 72071 \\
\hline Heavy Duty Trucks & Rigid $<=7,5 \mathrm{t}$ & Conventional & 128930 & 73574 & 68192 & 61866 \\
\hline Heavy Duty Trucks & Rigid $<=7,5 \mathrm{t}$ & HD Euro I - 91/542/EEC Stage I & 57155 & 32648 & 30320 & 27886 \\
\hline Heavy Duty Trucks & Rigid $<=7,5 \mathrm{t}$ & HD Euro II - 91/542/EEC Stage II & 93847 & 53605 & 49779 & 45786 \\
\hline Heavy Duty Trucks & Rigid $<=7,5 \mathrm{t}$ & HD Euro III - 2000 Standards & 106664 & 60924 & 56580 & 52039 \\
\hline Heavy Duty Trucks & Rigid $<=7,5 \mathrm{t}$ & HD Euro IV - 2005 Standards & 43670 & 33946 & 32054 & 35423 \\
\hline Heavy Duty Trucks & Rigid $<=7,5 \mathrm{t}$ & HD Euro V - 2008 Standards & 0 & 7857 & 7780 & 13100 \\
\hline Heavy Duty Trucks & Rigid $<=7,5 \mathrm{t}$ & HD Euro VI & 0 & 0 & 0 & 0 \\
\hline Heavy Duty Trucks & Rigid 7,5-12t & Conventional & 58003 & 33099 & 30678 & 27834 \\
\hline Heavy Duty Trucks & Rigid $7,5-12 \mathrm{t}$ & HD Euro I - 91/542/EEC Stage I & 25715 & 14687 & 13641 & 12546 \\
\hline Heavy Duty Trucks & Rigid $7,5-12 \mathrm{t}$ & HD Euro II - 91/542/EEC Stage II & 42219 & 24116 & 22396 & 20599 \\
\hline Heavy Duty Trucks & Rigid $7,5-12 \mathrm{t}$ & HD Euro III - 2000 Standards & 47986 & 27410 & 25456 & 23414 \\
\hline Heavy Duty Trucks & Rigid $7,5-12 \mathrm{t}$ & HD Euro IV - 2005 Standards & 19646 & 15272 & 14421 & 15938 \\
\hline Heavy Duty Trucks & Rigid $7,5-12 \mathrm{t}$ & HD Euro V - 2008 Standards & 0 & 3537 & 3500 & 5893 \\
\hline Heavy Duty Trucks & Rigid $7,5-12 \mathrm{t}$ & HD Euro VI & 0 & 0 & 0 & 0 \\
\hline Heavy Duty Trucks & Rigid $12-14 \mathrm{t}$ & Conventional & 16229 & 9260 & 8584 & 7786 \\
\hline Heavy Duty Trucks & Rigid $12-14 \mathrm{t}$ & HD Euro I - 91/542/EEC Stage I & 7192 & 4109 & 3815 & 3510 \\
\hline Heavy Duty Trucks & Rigid $12-14 t$ & HD Euro II - 91/542/EEC Stage II & 11810 & 6747 & 6265 & 5763 \\
\hline Heavy Duty Trucks & Rigid $12-14 \mathrm{t}$ & HD Euro III - 2000 Standards & 13424 & 7668 & 7122 & 6548 \\
\hline Heavy Duty Trucks & Rigid $12-14 \mathrm{t}$ & HD Euro IV - 2005 Standards & 5496 & 4272 & 4035 & 4458 \\
\hline Heavy Duty Trucks & Rigid $12-14 \mathrm{t}$ & HD Euro V - 2008 Standards & 0 & 988 & 979 & 1649 \\
\hline Heavy Duty Trucks & Rigid $12-14 t$ & HD Euro VI & 0 & 0 & 0 & 0 \\
\hline Heavy Duty Trucks & Rigid $14-20 t$ & Conventional & 44696 & 25507 & 23641 & 21448 \\
\hline Heavy Duty Trucks & Rigid $14-20 t$ & HD Euro I - 91/542/EEC Stage I & 19815 & 11320 & 10511 & 9667 \\
\hline Heavy Duty Trucks & Rigid $14-20 t$ & HD Euro II - 91/542/EEC Stage II & 32534 & 18585 & 17257 & 15874 \\
\hline Heavy Duty Trucks & Rigid $14-20 t$ & HD Euro III - 2000 Standards & 36978 & 21124 & 19616 & 18042 \\
\hline Heavy Duty Trucks & Rigid $14-20 t$ & HD Euro IV - 2005 Standards & 15141 & 11767 & 11111 & 12279 \\
\hline Heavy Duty Trucks & Rigid $14-20 t$ & HD Euro V - 2008 Standards & 0 & 2724 & 2698 & 4542 \\
\hline Heavy Duty Trucks & Rigid $14-20 t$ & HD Euro VI & 0 & 0 & 0 & 0 \\
\hline Heavy Duty Trucks & Rigid $20-26 \mathrm{t}$ & $\begin{array}{c}\text { Conventional } \\
\end{array}$ & 36329 & 20730 & 19215 & 17433 \\
\hline Heavy Duty Trucks & Rigid $20-26 \mathrm{t}$ & HD Euro I - 91/542/EEC Stage I & 16106 & 9200 & 8541 & 7859 \\
\hline Heavy Duty Trucks & Rigid $20-26 \mathrm{t}$ & HD Euro II - 91/542/EEC Stage II & 26440 & 15102 & 14026 & 12903 \\
\hline Heavy Duty Trucks & Rigid $20-26 \mathrm{t}$ & HD Euro III - 2000 Standards & 30051 & 17168 & 15941 & 14662 \\
\hline Heavy Duty Trucks & Rigid $20-26 \mathrm{t}$ & HD Euro IV - 2005 Standards & 12306 & 9564 & 9031 & 9981 \\
\hline Heavy Duty Trucks & Rigid $20-26 \mathrm{t}$ & HD Euro V - 2008 Standards & 0 & 2213 & 2193 & 3690 \\
\hline Heavy Duty Trucks & Rigid $20-26 \mathrm{t}$ & HD Euro VI & 0 & 0 & 0 & 0 \\
\hline Heavy Duty Trucks & Rigid $26-28 \mathrm{t}$ & Conventional & 92 & 54 & 49 & 46 \\
\hline Heavy Duty Trucks & Rigid $26-28 \mathrm{t}$ & HD Euro I - 91/542/EEC Stage I & 41 & 22 & 21 & 20 \\
\hline Heavy Duty Trucks & Rigid $26-28 \mathrm{t}$ & HD Euro II - 91/542/EEC Stage II & 66 & 38 & 37 & 35 \\
\hline Heavy Duty Trucks & Rigid $26-28 \mathrm{t}$ & HD Euro III - 2000 Standards & 77 & 45 & 40 & 38 \\
\hline Heavy Duty Trucks & Rigid $26-28 \mathrm{t}$ & HD Euro IV - 2005 Standards & 31 & 26 & 24 & 26 \\
\hline Heavy Duty Trucks & Rigid $26-28 \mathrm{t}$ & HD Euro V - 2008 Standards & 0 & 6 & 6 & 9 \\
\hline Heavy Duty Trucks & Rigid $26-28 \mathrm{t}$ & HD Euro VI & 0 & 0 & 0 & 0 \\
\hline Heavy Duty Trucks & Rigid $28-32 \mathrm{t}$ & Conventional & 3402 & 1941 & 1799 & 1632 \\
\hline Heavy Duty Trucks & Rigid $28-32 \mathrm{t}$ & HD Euro I - 91/542/EEC Stage I & 1507 & 860 & 798 & 736 \\
\hline Heavy Duty Trucks & Rigid $28-32 \mathrm{t}$ & HD Euro II - 91/542/EEC Stage II & 2477 & 1413 & 1312 & 1209 \\
\hline Heavy Duty Trucks & Rigid $28-32 \mathrm{t}$ & HD Euro III - 2000 Standards & 2815 & 1608 & 1493 & 1374 \\
\hline Heavy Duty Trucks & Rigid $28-32 \mathrm{t}$ & HD Euro IV - 2005 Standards & 1154 & 895 & 844 & 933 \\
\hline
\end{tabular}




\begin{tabular}{|c|c|c|c|c|c|c|c|c|c|}
\hline Sector & Subsector & Technology & 2008 & 2009 & 2010 & 2011 & 2012 & 2013 & 2014 \\
\hline Heavy Duty Trucks & Rigid $28-32 \mathrm{t}$ & HD Euro V - 2008 Standards & 0 & 208 & 205 & 345 & 391 & 444 & 442 \\
\hline Heavy Duty Trucks & Rigid $28-32 \mathrm{t}$ & HD Euro VI & 0 & 0 & 0 & 0 & 0 & 0 & 109 \\
\hline Heavy Duty Trucks & Rigid $>32 t$ & Conventional & 18762 & 10709 & 9924 & 9004 & 6459 & 5157 & 3995 \\
\hline Heavy Duty Trucks & Rigid $>32 t$ & HD Euro I - 91/542/EEC Stage I & 8321 & 4752 & 4411 & 4058 & 3000 & 2470 & 1998 \\
\hline Heavy Duty Trucks & Rigid $>32 t$ & HD Euro II - 91/542/EEC Stage II & 13659 & 7802 & 7244 & 6664 & 4925 & 4056 & 3281 \\
\hline Heavy Duty Trucks & Rigid $>32 t$ & HD Euro III - 2000 Standards & 15524 & 8867 & 8235 & 7575 & 5598 & 4610 & 3729 \\
\hline Heavy Duty Trucks & Rigid $>32 t$ & HD Euro IV - 2005 Standards & 6355 & 4940 & 4665 & 5157 & 4495 & 4258 & 3443 \\
\hline Heavy Duty Trucks & Rigid $>32 t$ & HD Euro V - 2008 Standards & 0 & 1145 & 1132 & 1907 & 2159 & 2444 & 2438 \\
\hline Heavy Duty Trucks & Rigid $>32 t$ & HD Euro VI & 0 & 0 & 0 & 0 & 0 & 0 & 607 \\
\hline Heavy Duty Trucks & Articulated $14-20 \mathrm{t}$ & Conventional & 7463 & 4218 & 3934 & 3626 & 2629 & 2123 & 1671 \\
\hline Heavy Duty Trucks & Articulated $14-20 \mathrm{t}$ & HD Euro I - 91/542/EEC Stage I & 1297 & 735 & 688 & 635 & 472 & 389 & 317 \\
\hline Heavy Duty Trucks & Articulated $14-20 \mathrm{t}$ & HD Euro II - 91/542/EEC Stage II & 1767 & 1007 & 942 & 873 & 647 & 536 & 435 \\
\hline Heavy Duty Trucks & Articulated $14-20 t$ & HD Euro III - 2000 Standards & 2835 & 1624 & 1523 & 1415 & 1053 & 874 & 713 \\
\hline Heavy Duty Trucks & Articulated $14-20 \mathrm{t}$ & HD Euro IV - 2005 Standards & 1640 & 1276 & 1184 & 1273 & 1102 & 1039 & 849 \\
\hline Heavy Duty Trucks & Articulated $14-20 \mathrm{t}$ & HD Euro V - 2008 Standards & 0 & 294 & 263 & 417 & 477 & 545 & 549 \\
\hline Heavy Duty Trucks & Articulated $14-20 t$ & HD Euro VI & 0 & 0 & 0 & 0 & 0 & 0 & 136 \\
\hline Heavy Duty Trucks & Articulated $20-28 \mathrm{t}$ & Conventional & 4894 & 2763 & 2579 & 2377 & 1724 & 1391 & 1095 \\
\hline Heavy Duty Trucks & Articulated $20-28 \mathrm{t}$ & HD Euro I - 91/542/EEC Stage I & 848 & 483 & 450 & 417 & 309 & 255 & 207 \\
\hline Heavy Duty Trucks & Articulated $20-28 \mathrm{t}$ & HD Euro II - 91/542/EEC Stage II & 1160 & 659 & 618 & 571 & 424 & 352 & 285 \\
\hline Heavy Duty Trucks & Articulated $20-28 \mathrm{t}$ & HD Euro III - 2000 Standards & 1859 & 1065 & 997 & 928 & 691 & 573 & 466 \\
\hline Heavy Duty Trucks & Articulated $20-28 \mathrm{t}$ & HD Euro IV - 2005 Standards & 1073 & 838 & 777 & 832 & 722 & 682 & 555 \\
\hline Heavy Duty Trucks & Articulated $20-28 \mathrm{t}$ & HD Euro V - 2008 Standards & 0 & 192 & 174 & 272 & 313 & 358 & 360 \\
\hline Heavy Duty Trucks & Articulated $20-28 \mathrm{t}$ & HD Euro VI & 0 & 0 & 0 & 0 & 0 & 0 & 89 \\
\hline Heavy Duty Trucks & Articulated $28-34 \mathrm{t}$ & Conventional & 3862 & 2181 & 2034 & 1876 & 1360 & 1099 & 864 \\
\hline Heavy Duty Trucks & Articulated $28-34 \mathrm{t}$ & HD Euro I - 91/542/EEC Stage I & 669 & 381 & 355 & 328 & 243 & 202 & 164 \\
\hline Heavy Duty Trucks & Articulated $28-34 \mathrm{t}$ & HD Euro II - 91/542/EEC Stage II & 914 & 521 & 486 & 449 & 336 & 277 & 225 \\
\hline Heavy Duty Trucks & Articulated $28-34 \mathrm{t}$ & HD Euro III - 2000 Standards & 1466 & 841 & 786 & 730 & 545 & 453 & 368 \\
\hline Heavy Duty Trucks & Articulated $28-34 \mathrm{t}$ & HD Euro IV - 2005 Standards & 848 & 659 & 612 & 658 & 570 & 537 & 438 \\
\hline Heavy Duty Trucks & Articulated $28-34 \mathrm{t}$ & HD Euro V - 2008 Standards & 0 & 153 & 138 & 215 & 247 & 283 & 284 \\
\hline Heavy Duty Trucks & Articulated $28-34 \mathrm{t}$ & HD Euro VI & 0 & 0 & 0 & 0 & 0 & 0 & 70 \\
\hline Heavy Duty Trucks & Articulated $34-40 \mathrm{t}$ & Conventional & 58846 & 33252 & 31011 & 28585 & 20740 & 16750 & 13170 \\
\hline Heavy Duty Trucks & Articulated $34-40 \mathrm{t}$ & HD Euro I - 91/542/EEC Stage I & 10216 & 5810 & 5421 & 5009 & 3720 & 3075 & 2496 \\
\hline Heavy Duty Trucks & Articulated $34-40 \mathrm{t}$ & HD Euro II - 91/542/EEC Stage II & 13945 & 7943 & 7425 & 6870 & 5106 & 4226 & 3434 \\
\hline Heavy Duty Trucks & Articulated $34-40 \mathrm{t}$ & HD Euro III - 2000 Standards & 22374 & 12800 & 12007 & 11149 & 8311 & 6899 & 5619 \\
\hline Heavy Duty Trucks & Articulated $34-40 \mathrm{t}$ & HD Euro IV - 2005 Standards & 12919 & 10063 & 9337 & 10027 & 8682 & 8192 & 6688 \\
\hline Heavy Duty Trucks & Articulated $34-40 \mathrm{t}$ & HD Euro V - 2008 Standards & 0 & 2325 & 2080 & 3284 & 3766 & 4301 & 4336 \\
\hline Heavy Duty Trucks & Articulated $34-40 \mathrm{t}$ & HD Euro VI & 0 & 0 & 0 & 0 & 0 & 0 & 1073 \\
\hline Heavy Duty Trucks & Articulated $40-50 \mathrm{t}$ & Conventional & 276 & 153 & 144 & 133 & 97 & 79 & 61 \\
\hline Heavy Duty Trucks & Articulated $40-50 \mathrm{t}$ & HD Euro I - 91/542/EEC Stage I & 46 & 26 & 24 & 23 & 18 & 15 & 11 \\
\hline Heavy Duty Trucks & Articulated $40-50 \mathrm{t}$ & HD Euro II - 91/542/EEC Stage II & 66 & 38 & 34 & 32 & 24 & 21 & 16 \\
\hline Heavy Duty Trucks & Articulated $40-50 \mathrm{t}$ & HD Euro III - 2000 Standards & 102 & 61 & 55 & 52 & 40 & 32 & 27 \\
\hline Heavy Duty Trucks & Articulated $40-50 \mathrm{t}$ & HD Euro IV - 2005 Standards & 61 & 48 & 43 & 46 & 40 & 37 & 31 \\
\hline Heavy Duty Trucks & Articulated $40-50 \mathrm{t}$ & HD Euro V - 2008 Standards & 0 & 10 & 9 & 14 & 18 & 21 & 20 \\
\hline Heavy Duty Trucks & Articulated $40-50 \mathrm{t}$ & HD Euro VI & 0 & 0 & 0 & 0 & 0 & 0 & 5 \\
\hline Heavy Duty Trucks & Articulated $50-60 \mathrm{t}$ & Conventional & 112 & 64 & 61 & 55 & 40 & 32 & 25 \\
\hline Heavy Duty Trucks & Articulated $50-60 \mathrm{t}$ & HD Euro I - 91/542/EEC Stage I & 20 & 13 & 9 & 9 & 7 & 6 & 5 \\
\hline Heavy Duty Trucks & Articulated $50-60 \mathrm{t}$ & HD Euro II - 91/542/EEC Stage II & 26 & 16 & 15 & 14 & 9 & 7 & 6 \\
\hline Heavy Duty Trucks & Articulated $50-60 \mathrm{t}$ & HD Euro III - 2000 Standards & 41 & 26 & 24 & 20 & 15 & 13 & 11 \\
\hline Heavy Duty Trucks & Articulated $50-60 \mathrm{t}$ & HD Euro IV - 2005 Standards & 26 & 19 & 18 & 20 & 18 & 15 & 12 \\
\hline Heavy Duty Trucks & Articulated $50-60 \mathrm{t}$ & HD Euro V - 2008 Standards & 0 & 3 & 3 & 6 & 7 & 7 & 8 \\
\hline Heavy Duty Trucks & Articulated $50-60 \mathrm{t}$ & HD Euro VI & 0 & 0 & 0 & 0 & 0 & 0 & 2 \\
\hline
\end{tabular}


Tragana Station

\begin{tabular}{|c|c|c|c|c|c|c|}
\hline Sector & Subsector & Technology & 2008 & 2009 & 2010 & 2011 \\
\hline Passenger Cars & Gasoline $0,8-1,4 \mid$ & ECE $15 / 02$ & 11017 & 4725 & 1688 & 0 \\
\hline Passenger Cars & Gasoline $0,8-1,4$ I & ECE 15/03 & 101792 & 71356 & 55713 & 40813 \\
\hline Passenger Cars & Gasoline $0,8-1,4$ I & ECE $15 / 04$ & 629056 & 477887 & 399834 & 308794 \\
\hline Passenger Cars & Gasoline $0,8-1,4$ I & PC Euro 1 - 91/441/EEC & 691890 & 553935 & 489818 & 400625 \\
\hline Passenger Cars & Gasoline $0,8-1,4$ I & PC Euro 2 - 94/12/EEC & 1118365 & 924465 & 848538 & 724626 \\
\hline Passenger Cars & Gasoline $0,8-1,4$ I & PC Euro 3 - 98/69/EC Stage2000 & 1171380 & 1041443 & 972366 & 847859 \\
\hline Passenger Cars & Gasoline $0,8-1,4 \mid$ & PC Euro 4 - 98/69/EC Stage2005 & 229326 & 245272 & 334764 & 362439 \\
\hline Passenger Cars & Gasoline $0,8-1,4 \mid$ & PC Euro 5 - EC 715/2007 & 0 & 0 & 95615 & 152527 \\
\hline Passenger Cars & Gasoline $1,4-2,0$ I & ECE $15 / 02$ & 1590 & 607 & 200 & 0 \\
\hline Passenger Cars & Gasoline $1,4-2,0$ I & ECE 15/03 & 23028 & 15476 & 12051 & 8871 \\
\hline Passenger Cars & Gasoline $1,4-2,0$ I & ECE 15/04 & 164615 & 118798 & 97247 & 73360 \\
\hline Passenger Cars & Gasoline $1,4-2,0$ I & PC Euro 1 - 91/441/EEC & 294798 & 228331 & 201405 & 164221 \\
\hline Passenger Cars & Gasoline $1,4-2,0$ I & PC Euro 2 - 94/12/EEC & 457398 & 365427 & 334173 & 284034 \\
\hline Passenger Cars & Gasoline $1,4-2,0$ I & PC Euro 3 - 98/69/EC Stage2000 & 755488 & 659731 & 615443 & 535980 \\
\hline Passenger Cars & Gasoline $1,4-2,0$ I & PC Euro 4 - 98/69/EC Stage2005 & 171797 & 180737 & 212375 & 213529 \\
\hline Passenger Cars & Gasoline $1,4-2,0$ I & PC Euro 5 - EC 715/2007 & 0 & 0 & 39133 & 61531 \\
\hline Passenger Cars & Gasoline $>2,0$ I & ECE $15 / 02$ & 779 & 395 & 187 & 0 \\
\hline Passenger Cars & Gasoline $>2,0 \mathrm{I}$ & ECE $15 / 03$ & 1490 & 1077 & 969 & 815 \\
\hline Passenger Cars & Gasoline $>2,0 \mathrm{I}$ & ECE 15/04 & 2949 & 2147 & 1945 & 1648 \\
\hline Passenger Cars & Gasoline $>2,0 \mathrm{I}$ & PC Euro 1 - 91/441/EEC & 8394 & 6142 & 5590 & 4758 \\
\hline Passenger Cars & Gasoline $>2,0 \mathrm{I}$ & PC Euro 2 - 94/12/EEC & 12189 & 8981 & 8233 & 7054 \\
\hline Passenger Cars & Gasoline $>2,0 \mathrm{I}$ & PC Euro 3 - 98/69/EC Stage2000 & 51971 & 43704 & 40405 & 34906 \\
\hline Passenger Cars & Gasoline $>2,0 \mathrm{I}$ & PC Euro 4 - 98/69/EC Stage2005 & 16979 & 17115 & 17689 & 16375 \\
\hline Passenger Cars & Gasoline $>2,0 \mathrm{I}$ & PC Euro 5 - EC $715 / 2007$ & 0 & 0 & 1648 & 2465 \\
\hline Passenger Cars & Diesel $1,4-2,0$ I & Conventional & 2176 & 1174 & 591 & 260 \\
\hline Passenger Cars & Diesel 1,4-2,0 I & PC Euro 1 - 91/441/EEC & 12597 & 9100 & 6287 & 3889 \\
\hline Passenger Cars & Diesel 1,4 - 2,0 I & PC Euro 2 - 94/12/EEC & 11263 & 8266 & 5798 & 3635 \\
\hline Passenger Cars & Diesel $1,4-2,0$ I & PC Euro 3 - 98/69/EC Stage2000 & 33024 & 29869 & 25405 & 19739 \\
\hline Passenger Cars & Diesel $1,4-2,0$ I & PC Euro 4 - 98/69/EC Stage2005 & 4916 & 5548 & 9790 & 11744 \\
\hline Passenger Cars & Diesel $1,4-2,0$ I & PC Euro 5 - EC $715 / 2007$ & 0 & 0 & 4338 & 7161 \\
\hline Passenger Cars & Diesel $>2,0$ I & Conventional & 244 & 91 & 30 & 7 \\
\hline Passenger Cars & Diesel $>2,0$ I & PC Euro 1 - 91/441/EEC & 3308 & 2172 & 1430 & 811 \\
\hline Passenger Cars & Diesel $>2,0$ I & PC Euro 2 - 94/12/EEC & 2757 & 1859 & 1250 & 716 \\
\hline Passenger Cars & Diesel $>2,0 \mathrm{I}$ & PC Euro 3 - 98/69/EC Stage2000 & 12250 & 10944 & 9844 & 8107 \\
\hline Passenger Cars & Diesel $>2,0 \mathrm{I}$ & PC Euro 4 - 98/69/EC Stage2005 & 2375 & 2590 & 3522 & 3868 \\
\hline Passenger Cars & Diesel $>2,0$ I & PC Euro 5 - EC 715/2007 & 0 & 0 & 1010 & 1685 \\
\hline Passenger Cars & LPG & Conventional & 2 & 0 & 0 & 0 \\
\hline Passenger Cars & LPG & PC Euro 1 - 91/441/EEC & 42 & 9 & 2 & 0 \\
\hline Passenger Cars & LPG & PC Euro 2 - 94/12/EEC & 700 & 400 & 210 & 84 \\
\hline Passenger Cars & LPG & PC Euro 3 - 98/69/EC Stage2000 & 2797 & 2449 & 2125 & 1660 \\
\hline Passenger Cars & LPG & PC Euro 4 - 98/69/EC Stage2005 & 520 & 567 & 763 & 840 \\
\hline Passenger Cars & LPG & PC Euro 5 - EC 715/2007 & 0 & 0 & 214 & 363 \\
\hline Passenger Cars & 2-Stroke & Conventional & 0 & 0 & 42221 & 55116 \\
\hline Passenger Cars & Hybrid Gasoline 1,4-2,0I & PC Euro 4 - 98/69/EC Stage2005 & 0 & 0 & 48193 & 63072 \\
\hline Heavy Duty Trucks & Rigid $<=7,5 \mathrm{t}$ & Conventional & 100917 & 103348 & 112492 & 93630 \\
\hline Heavy Duty Trucks & Rigid $<=7,5 \mathrm{t}$ & HD Euro I - 91/542/EEC Stage I & 44737 & 45860 & 50016 & 42204 \\
\hline Heavy Duty Trucks & Rigid $<=7,5 \mathrm{t}$ & HD Euro II - 91/542/EEC Stage II & 73457 & 75298 & 82117 & 69295 \\
\hline Heavy Duty Trucks & Rigid $<=7,5 \mathrm{t}$ & HD Euro III - 2000 Standards & 83489 & 85579 & 93336 & 78758 \\
\hline Heavy Duty Trucks & Rigid $<=7,5 \mathrm{t}$ & HD Euro IV - 2005 Standards & 34182 & 47683 & 52878 & 53611 \\
\hline Heavy Duty Trucks & Rigid $<=7,5 \mathrm{t}$ & HD Euro V - 2008 Standards & 0 & 11036 & 12833 & 19826 \\
\hline Heavy Duty Trucks & Rigid $<=7,5 \mathrm{t}$ & HD Euro VI & 0 & 0 & 0 & 0 \\
\hline Heavy Duty Trucks & Rigid $7,5-12 \mathrm{t}$ & Conventional & 45401 & 46493 & 50607 & 42125 \\
\hline Heavy Duty Trucks & Rigid $7,5-12 \mathrm{t}$ & HD Euro I - 91/542/EEC Stage I & 20128 & 20630 & 22502 & 18988 \\
\hline Heavy Duty Trucks & Rigid $7,5-12 \mathrm{t}$ & HD Euro II - 91/542/EEC Stage II & 33046 & 33876 & 36946 & 31175 \\
\hline Heavy Duty Trucks & Rigid $7,5-12 \mathrm{t}$ & HD Euro III - 2000 Standards & 37560 & 38502 & 41992 & 35435 \\
\hline Heavy Duty Trucks & Rigid $7,5-12 \mathrm{t}$ & HD Euro IV - 2005 Standards & 15378 & 21452 & 23789 & 24121 \\
\hline Heavy Duty Trucks & Rigid $7,5-12 \mathrm{t}$ & HD Euro V - 2008 Standards & 0 & 4968 & 5773 & 8919 \\
\hline Heavy Duty Trucks & Rigid $7,5-12 \mathrm{t}$ & HD Euro VI & 0 & 0 & 0 & 0 \\
\hline Heavy Duty Trucks & Rigid $12-14 t$ & Conventional & 12703 & 13008 & 14161 & 11784 \\
\hline Heavy Duty Trucks & Rigid $12-14 \mathrm{t}$ & HD Euro I - 91/542/EEC Stage I & 5630 & 5772 & 6293 & 5313 \\
\hline Heavy Duty Trucks & Rigid $12-14 t$ & HD Euro II - 91/542/EEC Stage II & 9244 & 9477 & 10335 & 8722 \\
\hline Heavy Duty Trucks & Rigid $12-14 t$ & HD Euro III - 2000 Standards & 10508 & 10771 & 11748 & 9911 \\
\hline Heavy Duty Trucks & Rigid $12-14 t$ & HD Euro IV - 2005 Standards & 4302 & 6001 & 6656 & 6747 \\
\hline Heavy Duty Trucks & Rigid $12-14 \mathrm{t}$ & HD Euro V - 2008 Standards & 0 & 1388 & 1615 & 2496 \\
\hline Heavy Duty Trucks & Rigid $12-14 \mathrm{t}$ & HD Euro VI & 0 & 0 & 0 & 0 \\
\hline Heavy Duty Trucks & Rigid $14-20 t$ & Conventional & 34985 & 35830 & 39000 & 32460 \\
\hline Heavy Duty Trucks & Rigid $14-20 t$ & HD Euro I - 91/542/EEC Stage I & 15509 & 15900 & 17340 & 14631 \\
\hline Heavy Duty Trucks & Rigid $14-20 \mathrm{t}$ & HD Euro II - 91/542/EEC Stage II & 25465 & 26105 & 28467 & 24024 \\
\hline Heavy Duty Trucks & Rigid $14-20 t$ & HD Euro III - 2000 Standards & 28944 & 29672 & 32358 & 27306 \\
\hline Heavy Duty Trucks & Rigid $14-20 t$ & HD Euro IV - 2005 Standards & 11851 & 16529 & 18329 & 18584 \\
\hline Heavy Duty Trucks & Rigid $14-20 t$ & HD Euro V - 2008 Standards & 0 & 3827 & 4451 & 6875 \\
\hline Heavy Duty Trucks & Rigid $14-20 t$ & HD Euro VI & 0 & 0 & 0 & 0 \\
\hline Heavy Duty Trucks & Rigid $20-26 \mathrm{t}$ & Conventional & 28436 & 29119 & 31697 & 26384 \\
\hline Heavy Duty Trucks & Rigid $20-26 \mathrm{t}$ & HD Euro I - 91/542/EEC Stage I & 12607 & 12922 & 14090 & 11894 \\
\hline Heavy Duty Trucks & Rigid $20-26 \mathrm{t}$ & HD Euro II - 91/542/EEC Stage II & 20695 & 21214 & 23138 & 19527 \\
\hline Heavy Duty Trucks & Rigid $20-26 \mathrm{t}$ & HD Euro III - 2000 Standards & 23522 & 24116 & 26297 & 22190 \\
\hline Heavy Duty Trucks & Rigid $20-26 \mathrm{t}$ & HD Euro IV - 2005 Standards & 9632 & 13435 & 14897 & 15105 \\
\hline Heavy Duty Trucks & Rigid $20-26 \mathrm{t}$ & HD Euro V - 2008 Standards & 0 & 3108 & 3618 & 5585 \\
\hline Heavy Duty Trucks & Rigid $20-26 \mathrm{t}$ & HD Euro VI & 0 & 0 & 0 & 0 \\
\hline Heavy Duty Trucks & Rigid $26-28 \mathrm{t}$ & Conventional & 72 & 76 & 81 & 70 \\
\hline Heavy Duty Trucks & Rigid $26-28 \mathrm{t}$ & HD Euro I - 91/542/EEC Stage I & 32 & 31 & 35 & 31 \\
\hline Heavy Duty Trucks & Rigid $26-28 \mathrm{t}$ & HD Euro II - 91/542/EEC Stage II & 52 & 54 & 61 & 53 \\
\hline Heavy Duty Trucks & Rigid $26-28 \mathrm{t}$ & HD Euro III - 2000 Standards & 60 & 63 & 66 & 57 \\
\hline Heavy Duty Trucks & Rigid $26-28 \mathrm{t}$ & HD Euro IV - 2005 Standards & 24 & 36 & 40 & 39 \\
\hline Heavy Duty Trucks & Rigid $26-28 \mathrm{t}$ & HD Euro V - 2008 Standards & 0 & 9 & 10 & 13 \\
\hline Heavy Duty Trucks & Rigid $26-28 \mathrm{t}$ & HD Euro VI & 0 & 0 & 0 & 0 \\
\hline Heavy Duty Trucks & Rigid $28-32 \mathrm{t}$ & Conventional & 2663 & 2726 & 2967 & 2470 \\
\hline Heavy Duty Trucks & Rigid $28-32 \mathrm{t}$ & HD Euro I - 91/542/EEC Stage I & 1180 & 1208 & 1317 & 1114 \\
\hline Heavy Duty Trucks & Rigid $28-32 \mathrm{t}$ & HD Euro II - 91/542/EEC Stage II & 1939 & 1985 & 2165 & 1829 \\
\hline Heavy Duty Trucks & Rigid $28-32 \mathrm{t}$ & HD Euro III - 2000 Standards & 2203 & 2259 & 2463 & 2080 \\
\hline Heavy Duty Trucks & Rigid $28-32 \mathrm{t}$ & HD Euro IV - 2005 Standards & 904 & 1258 & 1393 & 1413 \\
\hline
\end{tabular}




\begin{tabular}{|c|c|c|c|c|c|c|c|c|c|}
\hline Sector & Subsector & Technology & 2008 & 2009 & 2010 & 2011 & 2012 & 2013 & 2014 \\
\hline Heavy Duty Trucks & Rigid $28-32 \mathrm{t}$ & HD Euro V - 2008 Standards & 0 & 292 & 338 & 522 & 650 & 794 & 801 \\
\hline Heavy Duty Trucks & Rigid $28-32 \mathrm{t}$ & HD Euro VI & 0 & 0 & 0 & 0 & 0 & 0 & 198 \\
\hline Heavy Duty Trucks & Rigid $>32 t$ & Conventional & 14686 & 15043 & 16371 & 13626 & 10751 & 9231 & 7247 \\
\hline Heavy Duty Trucks & Rigid $>32 t$ & HD Euro I - 91/542/EEC Stage I & 6513 & 6675 & 7277 & 6142 & 4993 & 4421 & 3625 \\
\hline Heavy Duty Trucks & Rigid $>32 \mathrm{t}$ & HD Euro II - 91/542/EEC Stage II & 10691 & 10960 & 11950 & 10086 & 8197 & 7260 & 5951 \\
\hline Heavy Duty Trucks & Rigid $>32 t$ & HD Euro III - 2000 Standards & 12151 & 12455 & 13585 & 11464 & 9318 & 8252 & 6763 \\
\hline Heavy Duty Trucks & Rigid $>32 t$ & HD Euro IV - 2005 Standards & 4974 & 6940 & 7696 & 7805 & 7481 & 7622 & 6245 \\
\hline Heavy Duty Trucks & Rigid $>32 t$ & HD Euro V - 2008 Standards & 0 & 1608 & 1867 & 2887 & 3593 & 4374 & 4423 \\
\hline Heavy Duty Trucks & Rigid $>32 t$ & HD Euro VI & 0 & 0 & 0 & 0 & 0 & 0 & 1101 \\
\hline Heavy Duty Trucks & Articulated $14-20 \mathrm{t}$ & Conventional & 5842 & 5924 & 6490 & 5488 & 4376 & 3801 & 3031 \\
\hline Heavy Duty Trucks & Articulated $14-20 \mathrm{t}$ & HD Euro I - 91/542/EEC Stage I & 1016 & 1033 & 1135 & 961 & 786 & 697 & 574 \\
\hline Heavy Duty Trucks & Articulated $14-20 \mathrm{t}$ & HD Euro II - 91/542/EEC Stage II & 1383 & 1415 & 1554 & 1321 & 1077 & 959 & 790 \\
\hline Heavy Duty Trucks & Articulated $14-20 \mathrm{t}$ & HD Euro III - 2000 Standards & 2219 & 2282 & 2513 & 2141 & 1753 & 1565 & 1293 \\
\hline Heavy Duty Trucks & Articulated $14-20 \mathrm{t}$ & HD Euro IV - 2005 Standards & 1283 & 1792 & 1953 & 1926 & 1833 & 1860 & 1539 \\
\hline Heavy Duty Trucks & Articulated $14-20 \mathrm{t}$ & HD Euro V - 2008 Standards & 0 & 413 & 434 & 632 & 794 & 975 & 996 \\
\hline Heavy Duty Trucks & Articulated $14-20 \mathrm{t}$ & HD Euro VI & 0 & 0 & 0 & 0 & 0 & 0 & 246 \\
\hline Heavy Duty Trucks & Articulated $20-28 \mathrm{t}$ & Conventional & 3830 & 3881 & 4254 & 3597 & 2870 & 2490 & 1987 \\
\hline Heavy Duty Trucks & Articulated $20-28 \mathrm{t}$ & HD Euro I - 91/542/EEC Stage I & 664 & 678 & 742 & 632 & 514 & 456 & 376 \\
\hline Heavy Duty Trucks & Articulated $20-28 \mathrm{t}$ & HD Euro II - 91/542/EEC Stage II & 908 & 925 & 1019 & 864 & 705 & 630 & 518 \\
\hline Heavy Duty Trucks & Articulated $20-28 \mathrm{t}$ & HD Euro III - 2000 Standards & 1455 & 1496 & 1645 & 1404 & 1150 & 1026 & 846 \\
\hline Heavy Duty Trucks & Articulated $20-28 \mathrm{t}$ & HD Euro IV - 2005 Standards & 840 & 1177 & 1282 & 1259 & 1202 & 1220 & 1007 \\
\hline Heavy Duty Trucks & Articulated $20-28 \mathrm{t}$ & HD Euro V - 2008 Standards & 0 & 269 & 288 & 412 & 522 & 640 & 654 \\
\hline Heavy Duty Trucks & Articulated $20-28 \mathrm{t}$ & HD Euro VI & 0 & 0 & 0 & 0 & 0 & 0 & 161 \\
\hline Heavy Duty Trucks & Articulated $28-34 \mathrm{t}$ & Conventional & 3023 & 3063 & 3356 & 2838 & 2263 & 1967 & 1568 \\
\hline Heavy Duty Trucks & Articulated $28-34 \mathrm{t}$ & HD Euro I - 91/542/EEC Stage I & 524 & 535 & 585 & 496 & 404 & 362 & 297 \\
\hline Heavy Duty Trucks & Articulated $28-34 \mathrm{t}$ & HD Euro II - 91/542/EEC Stage II & 716 & 732 & 802 & 680 & 558 & 496 & 407 \\
\hline Heavy Duty Trucks & Articulated $28-34 \mathrm{t}$ & HD Euro III - 2000 Standards & 1148 & 1181 & 1297 & 1106 & 908 & 811 & 668 \\
\hline Heavy Duty Trucks & Articulated $28-34 \mathrm{t}$ & HD Euro IV - 2005 Standards & 664 & 925 & 1009 & 996 & 948 & 962 & 795 \\
\hline Heavy Duty Trucks & Articulated $28-34 \mathrm{t}$ & HD Euro V - 2008 Standards & 0 & 216 & 227 & 325 & 412 & 506 & 515 \\
\hline Heavy Duty Trucks & Articulated $28-34 \mathrm{t}$ & HD Euro VI & 0 & 0 & 0 & 0 & 0 & 0 & 127 \\
\hline Heavy Duty Trucks & Articulated $34-40 \mathrm{t}$ & Conventional & 46061 & 46709 & 51157 & 43262 & 34520 & 29982 & 23889 \\
\hline Heavy Duty Trucks & Articulated $34-40 \mathrm{t}$ & HD Euro I - 91/542/EEC Stage I & 7997 & 8161 & 8942 & 7581 & 6191 & 5504 & 4528 \\
\hline Heavy Duty Trucks & Articulated $34-40 \mathrm{t}$ & HD Euro II - 91/542/EEC Stage II & 10915 & 11157 & 12248 & 10398 & 8499 & 7565 & 6228 \\
\hline Heavy Duty Trucks & Articulated $34-40 \mathrm{t}$ & HD Euro III - 2000 Standards & 17513 & 17980 & 19808 & 16873 & 13834 & 12348 & 10193 \\
\hline Heavy Duty Trucks & Articulated $34-40 \mathrm{t}$ & HD Euro IV - 2005 Standards & 10112 & 14135 & 15402 & 15175 & 14451 & 14664 & 12131 \\
\hline Heavy Duty Trucks & Articulated $34-40 \mathrm{t}$ & HD Euro V - 2008 Standards & 0 & 3265 & 3432 & 4971 & 6268 & 7699 & 7864 \\
\hline Heavy Duty Trucks & Articulated $34-40 \mathrm{t}$ & HD Euro VI & 0 & 0 & 0 & 0 & 0 & 0 & 1947 \\
\hline Heavy Duty Trucks & Articulated $40-50 \mathrm{t}$ & Conventional & 216 & 216 & 237 & 202 & 162 & 141 & 110 \\
\hline Heavy Duty Trucks & Articulated $40-50 \mathrm{t}$ & HD Euro I - 91/542/EEC Stage I & 36 & 36 & 40 & 35 & 29 & 27 & 20 \\
\hline Heavy Duty Trucks & Articulated $40-50 \mathrm{t}$ & HD Euro II - 91/542/EEC Stage II & 52 & 54 & 56 & 48 & 40 & 37 & 28 \\
\hline Heavy Duty Trucks & Articulated $40-50 \mathrm{t}$ & HD Euro III - 2000 Standards & 80 & 85 & 91 & 79 & 66 & 57 & 48 \\
\hline Heavy Duty Trucks & Articulated $40-50 \mathrm{t}$ & HD Euro IV - 2005 Standards & 48 & 67 & 71 & 70 & 66 & 67 & 57 \\
\hline Heavy Duty Trucks & Articulated $40-50 \mathrm{t}$ & HD Euro V - 2008 Standards & 0 & 13 & 15 & 22 & 29 & 37 & 37 \\
\hline Heavy Duty Trucks & Articulated $40-50 \mathrm{t}$ & HD Euro VI & 0 & 0 & 0 & 0 & 0 & 0 & 8 \\
\hline Heavy Duty Trucks & Articulated $50-60 \mathrm{t}$ & Conventional & 88 & 90 & 101 & 83 & 66 & 57 & 45 \\
\hline Heavy Duty Trucks & Articulated $50-60 \mathrm{t}$ & HD Euro I - 91/542/EEC Stage I & 16 & 18 & 15 & 13 & 11 & 10 & 8 \\
\hline Heavy Duty Trucks & Articulated $50-60 \mathrm{t}$ & HD Euro II - 91/542/EEC Stage II & 20 & 22 & 25 & 22 & 15 & 13 & 11 \\
\hline Heavy Duty Trucks & Articulated $50-60 \mathrm{t}$ & HD Euro III - 2000 Standards & 32 & 36 & 40 & 31 & 26 & 23 & 20 \\
\hline Heavy Duty Trucks & Articulated $50-60 \mathrm{t}$ & HD Euro IV - 2005 Standards & 20 & 27 & 30 & 31 & 29 & 27 & 23 \\
\hline Heavy Duty Trucks & Articulated $50-60 \mathrm{t}$ & HD Euro V - 2008 Standards & 0 & 4 & 5 & 9 & 11 & 13 & 14 \\
\hline Heavy Duty Trucks & Articulated $50-60 \mathrm{t}$ & HD Euro VI & 0 & 0 & 0 & 0 & 0 & 0 & 3 \\
\hline
\end{tabular}


Pelasgia Station

\begin{tabular}{|c|c|c|c|c|c|c|}
\hline Sector & Subsector & Technology & 2008 & 2009 & 2010 & 2011 \\
\hline Passenger Cars & Gasoline 0,8 - 1,4 I & ECE 15/02 & 7295 & 3806 & 1089 & 0 \\
\hline Passenger Cars & Gasoline $0,8-1,41$ & ECE 15/03 & 67401 & 57486 & 35941 & 26224 \\
\hline Passenger Cars & Gasoline $0,8-1,4$ I & ECE 15/04 & 416523 & 384993 & 257935 & 198418 \\
\hline Passenger Cars & Gasoline $0,8-1,4$ I & PC Euro 1 - 91/441/EEC & 458128 & 446259 & 315985 & 257424 \\
\hline Passenger Cars & Gasoline $0,8-1,4$ I & PC Euro 2 - 94/12/EEC & 740515 & 744764 & 547397 & 465613 \\
\hline Passenger Cars & Gasoline $0,8-1,4$ I & PC Euro 3 - 98/69/EC Stage2000 & 775618 & 839004 & 627280 & 544797 \\
\hline Passenger Cars & Gasoline $0,8-1,4$ I & PC Euro 4 - 98/69/EC Stage2005 & 151846 & 197595 & 215959 & 232887 \\
\hline Passenger Cars & Gasoline $0,8-1,4 \mathrm{I}$ & PC Euro 5 - EC 715/2007 & 0 & 0 & 61682 & 98007 \\
\hline Passenger Cars & Gasoline 1,4-2,0 I & ECE 15/02 & 1053 & 489 & 129 & 0 \\
\hline Passenger Cars & Gasoline 1,4-2,0 I & ECE 15/03 & 15248 & 12468 & 7774 & 5700 \\
\hline Passenger Cars & Gasoline $1,4-2,0$ I & ECE $15 / 04$ & 108998 & 95706 & 62735 & 47138 \\
\hline Passenger Cars & Gasoline 1,4-2,01 & PC Euro 1 - 91/441/EEC & 195198 & 183947 & 129928 & 105521 \\
\hline Passenger Cars & Gasoline 1,4-2,0 I & PC Euro 2 - 94/12/EEC & 302862 & 294394 & 215578 & 182508 \\
\hline Passenger Cars & Gasoline 1,4-2,0 I & PC Euro 3 - 98/69/EC Stage2000 & 500239 & 531491 & 397027 & 344397 \\
\hline Passenger Cars & Gasoline 1,4-2,0 I & PC Euro 4 - 98/69/EC Stage2005 & 113754 & 145605 & 137004 & 137204 \\
\hline Passenger Cars & Gasoline 1,4-2,01 & PC Euro 5 - EC 715/2007 & 0 & 0 & 25245 & 39537 \\
\hline Passenger Cars & Gasoline $>2,0$ I & ECE $15 / 02$ & 516 & 318 & 121 & 0 \\
\hline Passenger Cars & Gasoline $>2,0 \mathrm{I}$ & ECE 15/03 & 986 & 868 & 625 & 524 \\
\hline Passenger Cars & Gasoline $>2,0 \mathrm{I}$ & ECE 15/04 & 1952 & 1730 & 1255 & 1059 \\
\hline Passenger Cars & Gasoline $>2,0 \mathrm{I}$ & PC Euro 1 - 91/441/EEC & 5558 & 4948 & 3606 & 3057 \\
\hline Passenger Cars & Gasoline $>2,01$ & PC Euro 2 - 94/12/EEC & 8071 & 7235 & 5311 & 4532 \\
\hline Passenger Cars & Gasoline $>2,0$ I & PC Euro 3 - 98/69/EC Stage2000 & 34412 & 35209 & 26066 & 22429 \\
\hline Passenger Cars & Gasoline $>2,0$ I & $\begin{array}{l}\text { PC Euro } 4 \text { - 98/69/EC Stage2005 } \\
\end{array}$ & 11243 & 13788 & 11411 & 10522 \\
\hline Passenger Cars & Gasoline $>2,0 \mathrm{I}$ & PC Euro 5 - EC 715/2007 & 0 & 0 & 1063 & 1584 \\
\hline Passenger Cars & Diesel $1,4-2,0$ I & Conventional & 1441 & 946 & 382 & 167 \\
\hline Passenger Cars & Diesel $1,4-2,0$ I & PC Euro 1 - 91/441/EEC & 8341 & 7331 & 4056 & 2499 \\
\hline Passenger Cars & Diesel $1,4-2,0$ I & PC Euro 2 - 94/12/EEC & 7457 & 6659 & 3740 & 2336 \\
\hline Passenger Cars & Diesel 1,4 - 2,0 I & PC Euro 3 - 98/69/EC Stage2000 & 21866 & 24063 & 16389 & 12683 \\
\hline Passenger Cars & Diesel $1,4-2,0$ I & PC Euro 4 - 98/69/EC Stage2005 & 3255 & 4470 & 6316 & 7546 \\
\hline Passenger Cars & Diesel $1,4-2,0$ I & PC Euro 5 - EC 715/2007 & 0 & 0 & 2799 & 4601 \\
\hline Passenger Cars & Diesel >2,0 I & Conventional & 162 & 73 & 19 & 5 \\
\hline Passenger Cars & Diesel $>2,0 \mathrm{I}$ & PC Euro 1 - 91/441/EEC & 2190 & 1750 & 922 & 521 \\
\hline Passenger Cars & Diesel $>2,0 \mathrm{I}$ & PC Euro 2 - 94/12/EEC & 1826 & 1498 & 806 & 460 \\
\hline Passenger Cars & Diesel $>2,0 \mathrm{I}$ & PC Euro 3 - 98/69/EC Stage2000 & 8112 & 8817 & 6351 & 5209 \\
\hline Passenger Cars & Diesel $>2,0$ I & PC Euro 4 - 98/69/EC Stage2005 & 1572 & 2086 & 2272 & 2485 \\
\hline Passenger Cars & Diesel $>2,0$ I & PC Euro 5 - EC 715/2007 & 0 & 0 & 652 & 1082 \\
\hline Passenger Cars & LPG & Conventional & 2 & 0 & 0 & 0 \\
\hline Passenger Cars & LPG & PC Euro 1 - 91/441/EEC & 27 & 7 & 1 & 0 \\
\hline Passenger Cars & LPG & PC Euro 2 - 94/12/EEC & 463 & 322 & 136 & 54 \\
\hline Passenger Cars & LPG & PC Euro 3 - 98/69/EC Stage2000 & 1852 & 1973 & 1371 & 1067 \\
\hline Passenger Cars & LPG & PC Euro 4 - 98/69/EC Stage2005 & 344 & 457 & 492 & 540 \\
\hline Passenger Cars & LPG & PC Euro 5 - EC 715/2007 & 0 & 0 & 138 & 233 \\
\hline Passenger Cars & 2-Stroke & Conventional & 0 & 0 & 27237 & 35415 \\
\hline Passenger Cars & Hybrid Gasoline $1,4-2,0$ I & PC Euro 4 - 98/69/EC Stage2005 & 0 & 0 & 31090 & 40527 \\
\hline Heavy Duty Trucks & Rigid $<=7,5 \mathrm{t}$ & Conventional & 139316 & 119041 & 94952 & 87223 \\
\hline Heavy Duty Trucks & Rigid $<=7,5 \mathrm{t}$ & HD Euro I - 91/542/EEC Stage I & 61759 & 52823 & 42217 & 39316 \\
\hline Heavy Duty Trucks & Rigid $<=7,5 \mathrm{t}$ & HD Euro II - 91/542/EEC Stage II & 101407 & 86731 & 69313 & 64553 \\
\hline Heavy Duty Trucks & Rigid $<=7,5 \mathrm{t}$ & HD Euro III - 2000 Standards & 115256 & 98574 & 78782 & 73368 \\
\hline Heavy Duty Trucks & Rigid $<=7,5 \mathrm{t}$ & HD Euro IV - 2005 Standards & 47188 & 54924 & 44633 & 49942 \\
\hline Heavy Duty Trucks & Rigid $<=7,5 \mathrm{t}$ & HD Euro V - 2008 Standards & 0 & 12712 & 10832 & 18469 \\
\hline Heavy Duty Trucks & Rigid $<=7,5 \mathrm{t}$ & HD Euro VI & 0 & 0 & 0 & 0 \\
\hline Heavy Duty Trucks & Rigid 7,5-12t & Conventional & 62676 & 53553 & 42716 & 39243 \\
\hline Heavy Duty Trucks & Rigid 7,5 - $12 \mathrm{t}$ & HD Euro I - 91/542/EEC Stage I & 27786 & 23763 & 18994 & 17688 \\
\hline Heavy Duty Trucks & Rigid $7,5-12 \mathrm{t}$ & HD Euro II - 91/542/EEC Stage II & 45620 & 39020 & 31185 & 29042 \\
\hline Heavy Duty Trucks & Rigid $7,5-12 \mathrm{t}$ & HD Euro III - 2000 Standards & 51852 & 44349 & 35445 & 33010 \\
\hline Heavy Duty Trucks & Rigid $7,5-12 \mathrm{t}$ & HD Euro IV - 2005 Standards & 21229 & 24709 & 20080 & 22470 \\
\hline Heavy Duty Trucks & Rigid $7,5-12 \mathrm{t}$ & HD Euro V - 2008 Standards & 0 & 5722 & 4873 & 8309 \\
\hline Heavy Duty Trucks & Rigid $7,5-12 \mathrm{t}$ & HD Euro VI & 0 & 0 & 0 & 0 \\
\hline Heavy Duty Trucks & Rigid $12-14 \mathrm{t}$ & Conventional & 17536 & 14983 & 11953 & 10977 \\
\hline Heavy Duty Trucks & Rigid $12-14 \mathrm{t}$ & HD Euro I - 91/542/EEC Stage I & 7772 & 6648 & 5312 & 4949 \\
\hline Heavy Duty Trucks & Rigid $12-14 \mathrm{t}$ & HD Euro II - 91/542/EEC Stage II & 12761 & 10916 & 8724 & 8125 \\
\hline Heavy Duty Trucks & Rigid $12-14 \mathrm{t}$ & HD Euro III - 2000 Standards & 14506 & 12406 & 9916 & 9232 \\
\hline Heavy Duty Trucks & Rigid $12-14 \mathrm{t}$ & HD Euro IV - 2005 Standards & 5939 & 6912 & 5618 & 6286 \\
\hline Heavy Duty Trucks & Rigid $12-14 \mathrm{t}$ & HD Euro V - 2008 Standards & 0 & 1599 & 1363 & 2325 \\
\hline Heavy Duty Trucks & Rigid $12-14 \mathrm{t}$ & HD Euro VI & 0 & 0 & 0 & 0 \\
\hline Heavy Duty Trucks & Rigid $14-20 t$ & Conventional & 48297 & 41270 & 32919 & 30239 \\
\hline Heavy Duty Trucks & Rigid $14-20 t$ & HD Euro I - 91/542/EEC Stage I & 21411 & 18315 & 14636 & 13630 \\
\hline Heavy Duty Trucks & Rigid $14-20 t$ & HD Euro II - 91/542/EEC Stage II & 35155 & 30069 & 24029 & 22380 \\
\hline Heavy Duty Trucks & Rigid $14-20 t$ & HD Euro III - 2000 Standards & 39957 & 34177 & 27313 & 25437 \\
\hline Heavy Duty Trucks & Rigid $14-20 t$ & HD Euro IV - 2005 Standards & 16360 & 19039 & 15471 & 17312 \\
\hline Heavy Duty Trucks & Rigid $14-20 t$ & HD Euro V - 2008 Standards & 0 & 4408 & 3757 & 6404 \\
\hline Heavy Duty Trucks & Rigid $14-20 t$ & HD Euro VI & 0 & 0 & 0 & 0 \\
\hline Heavy Duty Trucks & Rigid $20-26 \mathrm{t}$ & $\begin{array}{c}\text { Conventional } \\
\end{array}$ & 39256 & 33541 & 26755 & 24579 \\
\hline Heavy Duty Trucks & Rigid $20-26 \mathrm{t}$ & HD Euro I - 91/542/EEC Stage I & 17403 & 14885 & 11893 & 11080 \\
\hline Heavy Duty Trucks & Rigid $20-26 \mathrm{t}$ & HD Euro II - 91/542/EEC Stage II & 28570 & 24435 & 19531 & 18191 \\
\hline Heavy Duty Trucks & Rigid $20-26 \mathrm{t}$ & HD Euro III - 2000 Standards & 32472 & 27777 & 22197 & 20672 \\
\hline Heavy Duty Trucks & Rigid $20-26 \mathrm{t}$ & HD Euro IV - 2005 Standards & 13297 & 15474 & 12575 & 14071 \\
\hline Heavy Duty Trucks & Rigid $20-26 \mathrm{t}$ & HD Euro V - 2008 Standards & 0 & 3580 & 3054 & 5203 \\
\hline Heavy Duty Trucks & Rigid $20-26 \mathrm{t}$ & HD Euro VI & 0 & 0 & 0 & 0 \\
\hline Heavy Duty Trucks & Rigid $26-28 \mathrm{t}$ & Conventional & 99 & 88 & 68 & 65 \\
\hline Heavy Duty Trucks & Rigid $26-28 \mathrm{t}$ & HD Euro I - 91/542/EEC Stage I & 44 & 36 & 30 & 29 \\
\hline Heavy Duty Trucks & Rigid $26-28 \mathrm{t}$ & HD Euro II - 91/542/EEC Stage II & 72 & 62 & 51 & 49 \\
\hline Heavy Duty Trucks & Rigid $26-28 \mathrm{t}$ & HD Euro III - 2000 Standards & 83 & 72 & 55 & 53 \\
\hline Heavy Duty Trucks & Rigid $26-28 \mathrm{t}$ & HD Euro IV - 2005 Standards & 33 & 41 & 34 & 37 \\
\hline Heavy Duty Trucks & Rigid $26-28 \mathrm{t}$ & HD Euro V - 2008 Standards & 0 & 10 & 9 & 12 \\
\hline Heavy Duty Trucks & Rigid $26-28 \mathrm{t}$ & HD Euro VI & 0 & 0 & 0 & 0 \\
\hline Heavy Duty Trucks & Rigid $28-32 \mathrm{t}$ & Conventional & 3676 & 3140 & 2505 & 2301 \\
\hline Heavy Duty Trucks & Rigid $28-32 \mathrm{t}$ & HD Euro I - 91/542/EEC Stage I & 1628 & 1392 & 1112 & 1038 \\
\hline Heavy Duty Trucks & Rigid $28-32 \mathrm{t}$ & HD Euro II - 91/542/EEC Stage II & 2677 & 2287 & 1827 & 1704 \\
\hline Heavy Duty Trucks & Rigid $28-32 \mathrm{t}$ & HD Euro III - 2000 Standards & 3041 & 2602 & 2079 & 1937 \\
\hline Heavy Duty Trucks & Rigid $28-32 \mathrm{t}$ & HD Euro IV - 2005 Standards & 1247 & 1449 & 1176 & 1316 \\
\hline
\end{tabular}




\begin{tabular}{|c|c|c|c|c|c|c|c|c|c|}
\hline Sector & Subsector & Technology & 2008 & 2009 & 2010 & 2011 & 2012 & 2013 & 2014 \\
\hline Heavy Duty Trucks & Rigid $28-32 \mathrm{t}$ & HD Euro V - 2008 Standards & 0 & 336 & 285 & 486 & 661 & 853 & 886 \\
\hline Heavy Duty Trucks & Rigid $28-32 \mathrm{t}$ & HD Euro VI & 0 & 0 & 0 & 0 & 0 & 0 & 219 \\
\hline Heavy Duty Trucks & Rigid $>32 t$ & Conventional & 20274 & 17327 & 13818 & 12694 & 10928 & 9907 & 8016 \\
\hline Heavy Duty Trucks & Rigid $>32 \mathrm{t}$ & HD Euro I - 91/542/EEC Stage I & 8992 & 7688 & 6142 & 5722 & 5076 & 4745 & 4010 \\
\hline Heavy Duty Trucks & Rigid $>32 \mathrm{t}$ & HD Euro II - 91/542/EEC Stage II & 14760 & 12624 & 10087 & 9396 & 8333 & 7792 & 6583 \\
\hline Heavy Duty Trucks & Rigid $>32 t$ & HD Euro III - 2000 Standards & 16774 & 14347 & 11467 & 10679 & 9472 & 8857 & 7481 \\
\hline Heavy Duty Trucks & Rigid $>32 t$ & HD Euro IV - 2005 Standards & 6866 & 7993 & 6496 & 7271 & 7604 & 8180 & 6908 \\
\hline Heavy Duty Trucks & Rigid $>32 \mathrm{t}$ & HD Euro V - 2008 Standards & 0 & 1852 & 1576 & 2689 & 3653 & 4694 & 4892 \\
\hline Heavy Duty Trucks & Rigid $>32 t$ & HD Euro VI & 0 & 0 & 0 & 0 & 0 & 0 & 1218 \\
\hline Heavy Duty Trucks & Articulated $14-20 \mathrm{t}$ & Conventional & 8064 & 6824 & 5478 & 5113 & 4448 & 4079 & 3352 \\
\hline Heavy Duty Trucks & Articulated $14-20 \mathrm{t}$ & HD Euro I - 91/542/EEC Stage I & 1402 & 1190 & 958 & 895 & 799 & 748 & 635 \\
\hline Heavy Duty Trucks & Articulated $14-20 \mathrm{t}$ & HD Euro II - 91/542/EEC Stage II & 1910 & 1630 & 1312 & 1230 & 1094 & 1029 & 873 \\
\hline Heavy Duty Trucks & Articulated $14-20 t$ & HD Euro III - 2000 Standards & 3063 & 2628 & 2121 & 1994 & 1782 & 1680 & 1430 \\
\hline Heavy Duty Trucks & Articulated $14-20 \mathrm{t}$ & HD Euro IV - 2005 Standards & 1772 & 2064 & 1648 & 1794 & 1864 & 1997 & 1703 \\
\hline Heavy Duty Trucks & Articulated $14-20 \mathrm{t}$ & HD Euro V - 2008 Standards & 0 & 476 & 366 & 589 & 807 & 1047 & 1102 \\
\hline Heavy Duty Trucks & Articulated $14-20 \mathrm{t}$ & HD Euro VI & 0 & 0 & 0 & 0 & 0 & 0 & 272 \\
\hline Heavy Duty Trucks & Articulated $20-28 \mathrm{t}$ & Conventional & 5288 & 4470 & 3591 & 3351 & 2917 & 2673 & 2197 \\
\hline Heavy Duty Trucks & Articulated $20-28 \mathrm{t}$ & HD Euro I - 91/542/EEC Stage I & 916 & 781 & 626 & 589 & 523 & 489 & 416 \\
\hline Heavy Duty Trucks & Articulated $20-28 \mathrm{t}$ & HD Euro II - 91/542/EEC Stage II & 1253 & 1066 & 860 & 805 & 717 & 676 & 573 \\
\hline Heavy Duty Trucks & Articulated $20-28 \mathrm{t}$ & HD Euro III - 2000 Standards & 2009 & 1723 & 1389 & 1308 & 1169 & 1101 & 936 \\
\hline Heavy Duty Trucks & Articulated $20-28 \mathrm{t}$ & HD Euro IV - 2005 Standards & 1159 & 1356 & 1082 & 1173 & 1221 & 1309 & 1114 \\
\hline Heavy Duty Trucks & Articulated $20-28 \mathrm{t}$ & HD Euro V - 2008 Standards & 0 & 310 & 243 & 384 & 530 & 687 & 723 \\
\hline Heavy Duty Trucks & Articulated $20-28 \mathrm{t}$ & HD Euro VI & 0 & 0 & 0 & 0 & 0 & 0 & 178 \\
\hline Heavy Duty Trucks & Articulated $28-34 \mathrm{t}$ & Conventional & 4173 & 3528 & 2833 & 2644 & 2301 & 2112 & 1734 \\
\hline Heavy Duty Trucks & Articulated $28-34 \mathrm{t}$ & HD Euro I - 91/542/EEC Stage I & 723 & 616 & 494 & 462 & 411 & 389 & 329 \\
\hline Heavy Duty Trucks & Articulated $28-34 \mathrm{t}$ & HD Euro II - 91/542/EEC Stage II & 988 & 843 & 677 & 633 & 568 & 532 & 451 \\
\hline Heavy Duty Trucks & Articulated $28-34 \mathrm{t}$ & HD Euro III - 2000 Standards & 1584 & 1361 & 1095 & 1030 & 923 & 871 & 739 \\
\hline Heavy Duty Trucks & Articulated $28-34 \mathrm{t}$ & HD Euro IV - 2005 Standards & 916 & 1066 & 852 & 928 & 964 & 1032 & 880 \\
\hline Heavy Duty Trucks & Articulated $28-34 \mathrm{t}$ & HD Euro V - 2008 Standards & 0 & 248 & 192 & 302 & 418 & 543 & 570 \\
\hline Heavy Duty Trucks & Articulated $28-34 \mathrm{t}$ & HD Euro VI & 0 & 0 & 0 & 0 & 0 & 0 & 141 \\
\hline Heavy Duty Trucks & Articulated $34-40 t$ & Conventional & 63586 & 53801 & 43180 & 40301 & 35090 & 32178 & 26425 \\
\hline Heavy Duty Trucks & Articulated $34-40 t$ & HD Euro I - 91/542/EEC Stage I & 11039 & 9401 & 7548 & 7062 & 6293 & 5907 & 5008 \\
\hline Heavy Duty Trucks & Articulated $34-40 \mathrm{t}$ & HD Euro II - 91/542/EEC Stage II & 15069 & 12851 & 10338 & 9686 & 8639 & 8119 & 6889 \\
\hline Heavy Duty Trucks & Articulated $34-40 \mathrm{t}$ & HD Euro III - 2000 Standards & 24176 & 20710 & 16719 & 15718 & 14062 & 13253 & 11275 \\
\hline Heavy Duty Trucks & Articulated $34-40 t$ & HD Euro IV - 2005 Standards & 13959 & 16282 & 13000 & 14137 & 14689 & 15738 & 13419 \\
\hline Heavy Duty Trucks & Articulated $34-40 \mathrm{t}$ & HD Euro V - 2008 Standards & 0 & 3761 & 2897 & 4630 & 6372 & 8263 & 8699 \\
\hline Heavy Duty Trucks & Articulated $34-40 t$ & HD Euro VI & 0 & 0 & 0 & 0 & 0 & 0 & 2154 \\
\hline Heavy Duty Trucks & Articulated $40-50 \mathrm{t}$ & Conventional & 298 & 248 & 200 & 188 & 164 & 151 & 122 \\
\hline Heavy Duty Trucks & Articulated $40-50 \mathrm{t}$ & HD Euro I - 91/542/EEC Stage I & 50 & 41 & 34 & 33 & 30 & 29 & 22 \\
\hline Heavy Duty Trucks & Articulated $40-50 \mathrm{t}$ & HD Euro II - 91/542/EEC Stage II & 72 & 62 & 47 & 45 & 41 & 40 & 31 \\
\hline Heavy Duty Trucks & Articulated $40-50 \mathrm{t}$ & HD Euro III - 2000 Standards & 110 & 98 & 77 & 74 & 67 & 61 & 53 \\
\hline Heavy Duty Trucks & Articulated $40-50 \mathrm{t}$ & HD Euro IV - 2005 Standards & 66 & 78 & 60 & 65 & 67 & 72 & 63 \\
\hline Heavy Duty Trucks & Articulated $40-50 \mathrm{t}$ & HD Euro V - 2008 Standards & 0 & 16 & 13 & 20 & 30 & 40 & 41 \\
\hline Heavy Duty Trucks & Articulated $40-50 \mathrm{t}$ & HD Euro VI & 0 & 0 & 0 & 0 & 0 & 0 & 9 \\
\hline Heavy Duty Trucks & Articulated $50-60 \mathrm{t}$ & Conventional & 121 & 103 & 85 & 78 & 67 & 61 & 50 \\
\hline Heavy Duty Trucks & Articulated $50-60 \mathrm{t}$ & HD Euro I - 91/542/EEC Stage I & 22 & 21 & 13 & 12 & 11 & 11 & 9 \\
\hline Heavy Duty Trucks & Articulated $50-60 \mathrm{t}$ & HD Euro II - 91/542/EEC Stage II & 28 & 26 & 21 & 20 & 15 & 14 & 13 \\
\hline Heavy Duty Trucks & Articulated $50-60 \mathrm{t}$ & HD Euro III - 2000 Standards & 44 & 41 & 34 & 29 & 26 & 25 & 22 \\
\hline Heavy Duty Trucks & Articulated $50-60 \mathrm{t}$ & HD Euro IV - 2005 Standards & 28 & 31 & 26 & 29 & 30 & 29 & 25 \\
\hline Heavy Duty Trucks & Articulated $50-60 \mathrm{t}$ & HD Euro V - 2008 Standards & 0 & 5 & 4 & 8 & 11 & 14 & 16 \\
\hline Heavy Duty Trucks & Articulated $50-60 \mathrm{t}$ & HD Euro VI & 0 & 0 & 0 & 0 & 0 & 0 & 3 \\
\hline
\end{tabular}


Moshohori Station

\begin{tabular}{|c|c|c|c|c|c|c|}
\hline Sector & Subsector & Technology & 2008 & 2009 & 2010 & 2011 \\
\hline Passenger Cars & Gasoline $0,8-1,4 \mathrm{I}$ & ECE 15/02 & 8043 & 4101 & 1138 & 0 \\
\hline Passenger Cars & Gasoline $0,8-1,4$ I & ECE 15/03 & 74310 & 61942 & 37557 & 26541 \\
\hline Passenger Cars & Gasoline $0,8-1,4$ I & ECE $15 / 04$ & 459222 & 414840 & 269533 & 200815 \\
\hline Passenger Cars & Gasoline $0,8-1,4$ I & PC Euro 1 - 91/441/EEC & 505092 & 480855 & 330192 & 260535 \\
\hline Passenger Cars & Gasoline $0,8-1,4 \mathrm{I}$ & PC Euro 2 - 94/12/EEC & 816426 & 802502 & 572010 & 471240 \\
\hline Passenger Cars & Gasoline $0,8-1,4 \mid$ & PC Euro 3 - 98/69/EC Stage2000 & 855128 & 904047 & 655484 & 551381 \\
\hline Passenger Cars & Gasoline $0,8-1,4 \mathrm{I}$ & PC Euro 4 - 98/69/EC Stage2005 & 167412 & 212914 & 225669 & 235702 \\
\hline Passenger Cars & Gasoline $0,8-1,4 \mathrm{I}$ & PC Euro 5 - EC 715/2007 & 0 & 0 & 64455 & 99191 \\
\hline Passenger Cars & Gasoline 1,4-2,0 I & ECE $15 / 02$ & 1161 & 527 & 135 & 0 \\
\hline Passenger Cars & Gasoline $1,4-2,0$ I & ECE 15/03 & 16811 & 13434 & 8124 & 5769 \\
\hline Passenger Cars & Gasoline 1,4-2,0 I & ECE 15/04 & 120172 & 103125 & 65555 & 47708 \\
\hline Passenger Cars & Gasoline 1,4-2,0 I & PC Euro 1 - 91/441/EEC & 215208 & 198208 & 135770 & 106796 \\
\hline Passenger Cars & Gasoline $1,4-2,0$ I & PC Euro 2 - 94/12/EEC & 333909 & 317217 & 225270 & 184713 \\
\hline Passenger Cars & Gasoline 1,4 - 2,0 I & PC Euro 3 - 98/69/EC Stage2000 & 551520 & 572694 & 414878 & 348559 \\
\hline Passenger Cars & Gasoline $1,4-2,0$ I & PC Euro 4 - 98/69/EC Stage2005 & 125415 & 156892 & 143164 & 138862 \\
\hline Passenger Cars & Gasoline 1,4-2,0 I & PC Euro 5 - EC 715/2007 & 0 & 0 & 26380 & 40015 \\
\hline Passenger Cars & Gasoline $>2,0$ I & ECE $15 / 02$ & 569 & 343 & 126 & 0 \\
\hline Passenger Cars & Gasoline $>2,0 \mathrm{I}$ & ECE 15/03 & 1087 & 935 & 653 & 530 \\
\hline Passenger Cars & Gasoline $>2,0 \mathrm{I}$ & ECE $15 / 04$ & 2153 & 1864 & 1311 & 1072 \\
\hline Passenger Cars & Gasoline $>2,0 \mathrm{I}$ & PC Euro 1 - 91/441/EEC & 6128 & 5331 & 3768 & 3094 \\
\hline Passenger Cars & Gasoline $>2,0$ I & PC Euro 2 - 94/12/EEC & 8898 & 7796 & 5550 & 4587 \\
\hline Passenger Cars & Gasoline $>2,0 \mathrm{I}$ & PC Euro 3 - 98/69/EC Stage2000 & 37940 & 37938 & 27238 & 22700 \\
\hline Passenger Cars & Gasoline $>2,0 \mathrm{I}$ & PC Euro 4 - 98/69/EC Stage2005 & 12395 & 14857 & 11925 & 10649 \\
\hline Passenger Cars & Gasoline $>2,0$ I & PC Euro 5 - EC 715/2007 & 0 & 0 & 1111 & 1603 \\
\hline Passenger Cars & Diesel $1,4-2,0$ I & Conventional & 1588 & 1019 & 399 & 169 \\
\hline Passenger Cars & Diesel $1,4-2,0$ I & PC Euro 1 - 91/441/EEC & 9196 & 7899 & 4238 & 2529 \\
\hline Passenger Cars & Diesel 1,4 - 2,0 I & PC Euro 2 - 94/12/EEC & 8222 & 7175 & 3909 & 2364 \\
\hline Passenger Cars & Diesel 1,4 - 2,0 I & PC Euro 3 - 98/69/EC Stage2000 & 24108 & 25929 & 17125 & 12836 \\
\hline Passenger Cars & Diesel 1,4 - 2,0 I & PC Euro 4 - 98/69/EC Stage2005 & 3589 & 4816 & 6600 & 7638 \\
\hline Passenger Cars & Diesel 1,4-2,0 I & PC Euro 5 - EC 715/2007 & 0 & 0 & 2924 & 4657 \\
\hline Passenger Cars & Diesel $>2,0$ I & Conventional & 178 & 79 & 20 & 5 \\
\hline Passenger Cars & Diesel >2,0 I & PC Euro 1 - 91/441/EEC & 2415 & 1886 & 964 & 527 \\
\hline Passenger Cars & Diesel $>2,0$ I & PC Euro 2 - 94/12/EEC & 2013 & 1614 & 842 & 466 \\
\hline Passenger Cars & Diesel $>2,0$ I & PC Euro 3 - 98/69/EC Stage2000 & 8943 & 9500 & 6636 & 5272 \\
\hline Passenger Cars & Diesel $>2,0$ I & PC Euro 4 - 98/69/EC Stage2005 & 1734 & 2248 & 2374 & 2515 \\
\hline Passenger Cars & Diesel $>2,0$ I & PC Euro 5 - EC 715/2007 & 0 & 0 & 681 & 1096 \\
\hline Passenger Cars & LPG & Conventional & 2 & 0 & 0 & 0 \\
\hline Passenger Cars & LPG & PC Euro 1 - 91/441/EEC & 30 & 8 & 1 & 0 \\
\hline Passenger Cars & LPG & PC Euro 2 - 94/12/EEC & 511 & 347 & 142 & 55 \\
\hline Passenger Cars & LPG & PC Euro 3 - 98/69/EC Stage2000 & 2042 & 2126 & 1433 & 1079 \\
\hline Passenger Cars & LPG & PC Euro 4 - 98/69/EC Stage2005 & 380 & 493 & 515 & 546 \\
\hline Passenger Cars & LPG & PC Euro 5 - EC $715 / 2007$ & 0 & 0 & 144 & 236 \\
\hline Passenger Cars & 2-Stroke & Conventional & 0 & 0 & 28461 & 35843 \\
\hline Passenger Cars & Hybrid Gasoline $<1,4 \mathrm{I}$ & PC Euro 4 - 98/69/EC Stage2005 & 0 & 0 & 0 & 0 \\
\hline Passenger Cars & Hybrid Gasoline $1,4-2,0$ I & PC Euro 4 - 98/69/EC Stage2005 & 0 & 0 & 32488 & 41017 \\
\hline Heavy Duty Trucks & Rigid $<=7,5 \mathrm{t}$ & Conventional & 89644 & 66692 & 50443 & 51452 \\
\hline Heavy Duty Trucks & Rigid $<=7,5 \mathrm{t}$ & HD Euro I - 91/542/EEC Stage I & 39740 & 29594 & 22428 & 23192 \\
\hline Heavy Duty Trucks & Rigid $<=7,5 \mathrm{t}$ & HD Euro II - 91/542/EEC Stage II & 65251 & 48591 & 36822 & 38079 \\
\hline Heavy Duty Trucks & Rigid $<=7,5 \mathrm{t}$ & HD Euro III - 2000 Standards & 74163 & 55225 & 41853 & 43279 \\
\hline Heavy Duty Trucks & Rigid $<=7,5 \mathrm{t}$ & HD Euro IV - 2005 Standards & 30363 & 30771 & 23711 & 29460 \\
\hline Heavy Duty Trucks & Rigid $<=7,5 \mathrm{t}$ & HD Euro V - 2008 Standards & 0 & 7122 & 5755 & 10895 \\
\hline Heavy Duty Trucks & Rigid $<=7,5 \mathrm{t}$ & HD Euro VI & 0 & 0 & 0 & 0 \\
\hline Heavy Duty Trucks & Rigid $7,5-12 \mathrm{t}$ & Conventional & 40329 & 30003 & 22692 & 23149 \\
\hline Heavy Duty Trucks & Rigid $7,5-12 \mathrm{t}$ & HD Euro I - 91/542/EEC Stage I & 17879 & 13313 & 10090 & 10434 \\
\hline Heavy Duty Trucks & Rigid $7,5-12 \mathrm{t}$ & HD Euro II - 91/542/EEC Stage II & 29355 & 21861 & 16567 & 17131 \\
\hline Heavy Duty Trucks & Rigid $7,5-12 \mathrm{t}$ & HD Euro III - 2000 Standards & 33364 & 24846 & 18830 & 19472 \\
\hline Heavy Duty Trucks & Rigid $7,5-12 \mathrm{t}$ & HD Euro IV - 2005 Standards & 13660 & 13843 & 10667 & 13255 \\
\hline Heavy Duty Trucks & Rigid $7,5-12 \mathrm{t}$ & HD Euro V - 2008 Standards & 0 & 3206 & 2589 & 4901 \\
\hline Heavy Duty Trucks & Rigid $7,5-12 \mathrm{t}$ & HD Euro VI & 0 & 0 & 0 & 0 \\
\hline Heavy Duty Trucks & Rigid $12-14 \mathrm{t}$ & Conventional & 11284 & 8394 & 6350 & 6476 \\
\hline Heavy Duty Trucks & Rigid $12-14 \mathrm{t}$ & HD Euro I - 91/542/EEC Stage I & 5001 & 3725 & 2822 & 2920 \\
\hline Heavy Duty Trucks & Rigid $12-14 t$ & HD Euro II - 91/542/EEC Stage II & 8211 & 6116 & 4634 & 4793 \\
\hline Heavy Duty Trucks & Rigid $12-14 t$ & HD Euro III - 2000 Standards & 9334 & 6951 & 5268 & 5446 \\
\hline Heavy Duty Trucks & Rigid $12-14 \mathrm{t}$ & HD Euro IV - 2005 Standards & 3822 & 3872 & 2985 & 3708 \\
\hline Heavy Duty Trucks & Rigid $12-14 t$ & HD Euro V - 2008 Standards & 0 & 896 & 724 & 1372 \\
\hline Heavy Duty Trucks & Rigid $12-14 \mathrm{t}$ & HD Euro VI & 0 & 0 & 0 & 0 \\
\hline Heavy Duty Trucks & Rigid $14-20 t$ & Conventional & 31077 & 23121 & 17488 & 17838 \\
\hline Heavy Duty Trucks & Rigid $14-20 \mathrm{t}$ & HD Euro I - 91/542/EEC Stage I & 13777 & 10261 & 7775 & 8040 \\
\hline Heavy Duty Trucks & Rigid $14-20 t$ & HD Euro II - 91/542/EEC Stage II & 22621 & 16846 & 12765 & 13202 \\
\hline Heavy Duty Trucks & Rigid $14-20 t$ & HD Euro III - 2000 Standards & 25711 & 19148 & 14510 & 15005 \\
\hline Heavy Duty Trucks & Rigid $14-20 t$ & HD Euro IV - 2005 Standards & 10527 & 10667 & 8219 & 10212 \\
\hline Heavy Duty Trucks & Rigid $14-20 t$ & HD Euro V - 2008 Standards & 0 & 2470 & 1996 & 3778 \\
\hline Heavy Duty Trucks & Rigid $14-20 t$ & HD Euro VI & 0 & 0 & 0 & 0 \\
\hline Heavy Duty Trucks & Rigid $20-26 \mathrm{t}$ & Conventional & 25260 & 18791 & 14213 & 14499 \\
\hline Heavy Duty Trucks & Rigid $20-26 \mathrm{t}$ & HD Euro I - 91/542/EEC Stage I & 11198 & 8339 & 6318 & 6536 \\
\hline Heavy Duty Trucks & Rigid $20-26 \mathrm{t}$ & HD Euro II - 91/542/EEC Stage II & 18383 & 13690 & 10375 & 10731 \\
\hline Heavy Duty Trucks & Rigid $20-26 \mathrm{t}$ & HD Euro III - 2000 Standards & 20895 & 15562 & 11792 & 12194 \\
\hline Heavy Duty Trucks & Rigid $20-26 \mathrm{t}$ & HD Euro IV - 2005 Standards & 8556 & 8669 & 6680 & 8301 \\
\hline Heavy Duty Trucks & Rigid $20-26 \mathrm{t}$ & HD Euro V - 2008 Standards & 0 & 2006 & 1623 & 3069 \\
\hline Heavy Duty Trucks & Rigid $20-26 \mathrm{t}$ & HD Euro VI & 0 & 0 & 0 & 0 \\
\hline Heavy Duty Trucks & Rigid $26-28 \mathrm{t}$ & Conventional & 64 & 49 & 36 & 39 \\
\hline Heavy Duty Trucks & Rigid $26-28 \mathrm{t}$ & HD Euro I - 91/542/EEC Stage I & 28 & 20 & 16 & 17 \\
\hline Heavy Duty Trucks & Rigid $26-28 \mathrm{t}$ & HD Euro II - 91/542/EEC Stage II & 46 & 35 & 27 & 29 \\
\hline Heavy Duty Trucks & Rigid $26-28 \mathrm{t}$ & HD Euro III - 2000 Standards & 53 & 41 & 29 & 31 \\
\hline Heavy Duty Trucks & Rigid $26-28 \mathrm{t}$ & HD Euro IV - 2005 Standards & 21 & 23 & 18 & 22 \\
\hline Heavy Duty Trucks & Rigid $26-28 \mathrm{t}$ & HD Euro V - 2008 Standards & 0 & 6 & 5 & 7 \\
\hline Heavy Duty Trucks & Rigid $26-28 \mathrm{t}$ & HD Euro VI & 0 & 0 & 0 & 0 \\
\hline Heavy Duty Trucks & Rigid $28-32 \mathrm{t}$ & Conventional & 2365 & 1759 & 1331 & 1357 \\
\hline Heavy Duty Trucks & Rigid $28-32 \mathrm{t}$ & HD Euro I - 91/542/EEC Stage I & 1048 & 780 & 591 & 612 \\
\hline Heavy Duty Trucks & Rigid $28-32 \mathrm{t}$ & HD Euro II - 91/542/EEC Stage II & 1723 & 1281 & 971 & 1005 \\
\hline Heavy Duty Trucks & Rigid $28-32 \mathrm{t}$ & HD Euro III - 2000 Standards & 1957 & 1458 & 1104 & 1143 \\
\hline
\end{tabular}




\begin{tabular}{|c|c|c|c|c|c|c|c|c|c|}
\hline $\begin{array}{l}\text { Sector } \\
\end{array}$ & Subsector & Technology & 2008 & 2009 & 2010 & 2011 & 2012 & 2013 & 2014 \\
\hline Heavy Duty Trucks & Rigid $28-32 \mathrm{t}$ & HD Euro IV - 2005 Standards & 803 & 812 & 625 & 776 & 658 & 696 & 628 \\
\hline Heavy Duty Trucks & Rigid $28-32 \mathrm{t}$ & HD Euro V - 2008 Standards & 0 & 188 & 152 & 287 & 315 & 400 & 445 \\
\hline Heavy Duty Trucks & Rigid $28-32 \mathrm{t}$ & HD Euro VI & 0 & 0 & 0 & 0 & 0 & 0 & 110 \\
\hline Heavy Duty Trucks & Rigid $>32 t$ & Conventional & 13045 & 9707 & 7341 & 7488 & 5214 & 4651 & 4023 \\
\hline Heavy Duty Trucks & Rigid $>32 t$ & HD Euro I - 91/542/EEC Stage I & 5786 & 4307 & 3263 & 3375 & 2422 & 2228 & 2012 \\
\hline Heavy Duty Trucks & Rigid $>32 \mathrm{t}$ & HD Euro II - 91/542/EEC Stage II & 9497 & 7072 & 5359 & 5543 & 3976 & 3658 & 3304 \\
\hline Heavy Duty Trucks & Rigid $>32 t$ & HD Euro III - 2000 Standards & 10794 & 8038 & 6092 & 6300 & 4519 & 4158 & 3754 \\
\hline Heavy Duty Trucks & Rigid $>32 t$ & HD Euro IV - 2005 Standards & 4418 & 4478 & 3451 & 4289 & 3628 & 3840 & 3467 \\
\hline Heavy Duty Trucks & Rigid $>32 t$ & HD Euro V - 2008 Standards & 0 & 1038 & 837 & 1586 & 1743 & 2204 & 2455 \\
\hline Heavy Duty Trucks & Rigid $>32 t$ & HD Euro VI & 0 & 0 & 0 & 0 & 0 & 0 & 611 \\
\hline Heavy Duty Trucks & Articulated $14-20 \mathrm{t}$ & Conventional & 5189 & 3823 & 2910 & 3016 & 2122 & 1915 & 1682 \\
\hline Heavy Duty Trucks & Articulated $14-20 \mathrm{t}$ & HD Euro I - 91/542/EEC Stage I & 902 & 667 & 509 & 528 & 381 & 351 & 319 \\
\hline Heavy Duty Trucks & Articulated $14-20 \mathrm{t}$ & HD Euro II - 91/542/EEC Stage II & 1229 & 913 & 697 & 726 & 522 & 483 & 438 \\
\hline Heavy Duty Trucks & Articulated $14-20 \mathrm{t}$ & HD Euro III - 2000 Standards & 1971 & 1472 & 1127 & 1176 & 850 & 789 & 718 \\
\hline Heavy Duty Trucks & Articulated $14-20 \mathrm{t}$ & HD Euro IV - 2005 Standards & 1140 & 1157 & 876 & 1058 & 889 & 937 & 855 \\
\hline Heavy Duty Trucks & Articulated $14-20 \mathrm{t}$ & HD Euro V - 2008 Standards & 0 & 267 & 195 & 347 & 385 & 491 & 553 \\
\hline Heavy Duty Trucks & Articulated $14-20 \mathrm{t}$ & HD Euro VI & 0 & 0 & 0 & 0 & 0 & 0 & 137 \\
\hline Heavy Duty Trucks & Articulated $20-28 \mathrm{t}$ & Conventional & 3403 & 2504 & 1908 & 1977 & 1392 & 1255 & 1103 \\
\hline Heavy Duty Trucks & Articulated $20-28 \mathrm{t}$ & HD Euro I - 91/542/EEC Stage I & 590 & 438 & 333 & 347 & 249 & 230 & 209 \\
\hline Heavy Duty Trucks & Articulated $20-28 \mathrm{t}$ & HD Euro II - 91/542/EEC Stage II & 806 & 597 & 457 & 475 & 342 & 317 & 287 \\
\hline Heavy Duty Trucks & Articulated $20-28 \mathrm{t}$ & HD Euro III - 2000 Standards & 1293 & 965 & 738 & 771 & 558 & 517 & 470 \\
\hline Heavy Duty Trucks & Articulated $20-28 \mathrm{t}$ & HD Euro IV - 2005 Standards & 746 & 759 & 575 & 692 & 583 & 615 & 559 \\
\hline Heavy Duty Trucks & Articulated $20-28 \mathrm{t}$ & HD Euro V - 2008 Standards & 0 & 174 & 129 & 227 & 253 & 323 & 363 \\
\hline Heavy Duty Trucks & Articulated $20-28 \mathrm{t}$ & HD Euro VI & 0 & 0 & 0 & 0 & 0 & 0 & 90 \\
\hline Heavy Duty Trucks & Articulated $28-34 \mathrm{t}$ & Conventional & 2685 & 1977 & 1505 & 1560 & 1098 & 991 & 870 \\
\hline Heavy Duty Trucks & Articulated $28-34 \mathrm{t}$ & HD Euro I - 91/542/EEC Stage I & 465 & 345 & 262 & 272 & 196 & 182 & 165 \\
\hline Heavy Duty Trucks & Articulated $28-34 \mathrm{t}$ & HD Euro II - 91/542/EEC Stage II & 636 & 472 & 360 & 374 & 271 & 250 & 226 \\
\hline Heavy Duty Trucks & Articulated $28-34 \mathrm{t}$ & HD Euro III - 2000 Standards & 1019 & 762 & 582 & 608 & 440 & 409 & 371 \\
\hline Heavy Duty Trucks & Articulated $28-34 \mathrm{t}$ & HD Euro IV - 2005 Standards & 590 & 597 & 453 & 547 & 460 & 485 & 441 \\
\hline Heavy Duty Trucks & Articulated $28-34 \mathrm{t}$ & HD Euro V - 2008 Standards & 0 & 139 & 102 & 178 & 200 & 255 & 286 \\
\hline Heavy Duty Trucks & Articulated $28-34 \mathrm{t}$ & HD Euro VI & 0 & 0 & 0 & 0 & 0 & 0 & 71 \\
\hline Heavy Duty Trucks & Articulated $34-40 \mathrm{t}$ & Conventional & 40915 & 30142 & 22939 & 23773 & 16742 & 15106 & 13261 \\
\hline Heavy Duty Trucks & Articulated $34-40 \mathrm{t}$ & HD Euro I - 91/542/EEC Stage I & 7103 & 5267 & 4010 & 4166 & 3003 & 2773 & 2513 \\
\hline Heavy Duty Trucks & Articulated $34-40 \mathrm{t}$ & HD Euro II - 91/542/EEC Stage II & 9696 & 7200 & 5492 & 5714 & 4122 & 3812 & 3457 \\
\hline Heavy Duty Trucks & Articulated $34-40 \mathrm{t}$ & HD Euro III - 2000 Standards & 15556 & 11603 & 8882 & 9272 & 6709 & 6222 & 5658 \\
\hline Heavy Duty Trucks & Articulated $34-40 \mathrm{t}$ & HD Euro IV - 2005 Standards & 8982 & 9122 & 6906 & 8339 & 7009 & 7389 & 6734 \\
\hline Heavy Duty Trucks & Articulated $34-40 \mathrm{t}$ & HD Euro V - 2008 Standards & 0 & 2107 & 1539 & 2731 & 3040 & 3879 & 4365 \\
\hline Heavy Duty Trucks & Articulated $34-40 \mathrm{t}$ & HD Euro VI & 0 & 0 & 0 & 0 & 0 & 0 & 1081 \\
\hline Heavy Duty Trucks & Articulated $40-50 \mathrm{t}$ & Conventional & 192 & 139 & 106 & 111 & 78 & 71 & 61 \\
\hline Heavy Duty Trucks & Articulated $40-50 \mathrm{t}$ & HD Euro I - 91/542/EEC Stage I & 32 & 23 & 18 & 19 & 14 & 14 & 11 \\
\hline Heavy Duty Trucks & Articulated $40-50 \mathrm{t}$ & HD Euro II - 91/542/EEC Stage II & 46 & 35 & 25 & 27 & 20 & 19 & 16 \\
\hline Heavy Duty Trucks & Articulated $40-50 \mathrm{t}$ & HD Euro III - 2000 Standards & 71 & 55 & 41 & 43 & 32 & 29 & 27 \\
\hline Heavy Duty Trucks & Articulated $40-50 \mathrm{t}$ & HD Euro IV - 2005 Standards & 43 & 43 & 32 & 39 & 32 & 34 & 31 \\
\hline Heavy Duty Trucks & Articulated $40-50 \mathrm{t}$ & HD Euro V - 2008 Standards & 0 & 9 & 7 & 12 & 14 & 19 & 20 \\
\hline Heavy Duty Trucks & Articulated $40-50 \mathrm{t}$ & HD Euro VI & 0 & 0 & 0 & 0 & 0 & 0 & 5 \\
\hline Heavy Duty Trucks & Articulated $50-60 \mathrm{t}$ & Conventional & 78 & 58 & 45 & 46 & 32 & 29 & 25 \\
\hline Heavy Duty Trucks & Articulated $50-60 \mathrm{t}$ & HD Euro I - 91/542/EEC Stage I & 14 & 12 & 7 & 7 & 5 & 5 & 5 \\
\hline Heavy Duty Trucks & Articulated $50-60 \mathrm{t}$ & HD Euro II - 91/542/EEC Stage II & 18 & 14 & 11 & 12 & 7 & 7 & 6 \\
\hline Heavy Duty Trucks & Articulated $50-60 \mathrm{t}$ & HD Euro III - 2000 Standards & 28 & 23 & 18 & 17 & 12 & 12 & 11 \\
\hline Heavy Duty Trucks & Articulated $50-60 \mathrm{t}$ & HD Euro IV - 2005 Standards & 18 & 17 & 14 & 17 & 14 & 14 & 13 \\
\hline Heavy Duty Trucks & Articulated $50-60 \mathrm{t}$ & HD Euro V - 2008 Standards & 0 & 3 & 2 & 5 & 5 & 7 & 8 \\
\hline Heavy Duty Trucks & Articulated $50-60 \mathrm{t}$ & HD Euro VI & 0 & 0 & 0 & 0 & 0 & 0 & 2 \\
\hline
\end{tabular}


Makrihori Station

\begin{tabular}{|c|c|c|c|c|c|c|}
\hline Sector & Subsector & Technology & 2008 & 2009 & 2010 & 2011 \\
\hline Passenger Cars & Gasoline $0,8-1,4$ I & ECE $15 / 02$ & 9347 & 4670 & 1322 & 0 \\
\hline Passenger Cars & Gasoline $0,8-1,4$ I & ECE $15 / 03$ & 86363 & 70532 & 43619 & 32872 \\
\hline Passenger Cars & Gasoline $0,8-1,4$ I & ECE $15 / 04$ & 533703 & 472366 & 313039 & 248712 \\
\hline Passenger Cars & Gasoline $0,8-1,4$ I & PC Euro 1 - 91/441/EEC & 587013 & 547536 & 383489 & 322675 \\
\hline Passenger Cars & Gasoline $0,8-1,4$ I & PC Euro 2 - 94/12/EEC & 948843 & 913785 & 664339 & 583635 \\
\hline Passenger Cars & Gasoline $0,8-1,4$ I & PC Euro 3 - 98/69/EC Stage2000 & 993822 & 1029412 & 761288 & 682890 \\
\hline Passenger Cars & Gasoline $0,8-1,4$ I & PC Euro 4 - 98/69/EC Stage2005 & 194565 & 242439 & 262094 & 291919 \\
\hline Passenger Cars & Gasoline $0,8-1,4$ I & PC Euro 5 - EC 715/2007 & 0 & 0 & 74859 & 122849 \\
\hline Passenger Cars & Gasoline $1,4-2,0$ I & ECE $15 / 02$ & 1349 & 600 & 157 & 0 \\
\hline Passenger Cars & Gasoline $1,4-2,0$ I & ECE $15 / 03$ & 19538 & 15297 & 9435 & 7145 \\
\hline Passenger Cars & Gasoline $1,4-2,0$ I & ECE 15/04 & 139662 & 117426 & 76137 & 59086 \\
\hline Passenger Cars & Gasoline $1,4-2,0$ I & PC Euro 1 - 91/441/EEC & 250113 & 225694 & 157685 & 132268 \\
\hline Passenger Cars & Gasoline $1,4-2,0$ I & PC Euro 2 - 94/12/EEC & 388066 & 361205 & 261632 & 228769 \\
\hline Passenger Cars & Gasoline $1,4-2,0$ I & PC Euro 3 - 98/69/EC Stage2000 & 640971 & 652110 & 481845 & 431694 \\
\hline Passenger Cars & Gasoline $1,4-2,0$ I & PC Euro 4 - 98/69/EC Stage2005 & 145756 & 178649 & 166273 & 171982 \\
\hline Passenger Cars & Gasoline $1,4-2,0$ I & PC Euro 5 - EC 715/2007 & 0 & 0 & 30638 & 49559 \\
\hline Passenger Cars & Gasoline $>2,0$ I & ECE $15 / 02$ & 661 & 390 & 146 & 0 \\
\hline Passenger Cars & Gasoline $>2,0$ I & ECE $15 / 03$ & 1264 & 1065 & 759 & 656 \\
\hline Passenger Cars & Gasoline $>2,0$ I & ECE 15/04 & 2502 & 2122 & 1523 & 1328 \\
\hline Passenger Cars & Gasoline $>2,0 \mathrm{I}$ & PC Euro 1 - 91/441/EEC & 7122 & 6071 & 4376 & 3832 \\
\hline Passenger Cars & Gasoline $>2,0 \mathrm{I}$ & PC Euro 2 - 94/12/EEC & 10342 & 8877 & 6445 & 5681 \\
\hline Passenger Cars & Gasoline $>2,0 \mathrm{I}$ & PC Euro 3 - 98/69/EC Stage2000 & 44093 & 43199 & 31634 & 28114 \\
\hline Passenger Cars & Gasoline $>2,0$ I & PC Euro 4 - 98/69/EC Stage2005 & 14406 & 16917 & 13849 & 13189 \\
\hline Passenger Cars & Gasoline $>2,0$ I & PC Euro 5 - EC 715/2007 & 0 & 0 & 1290 & 1985 \\
\hline Passenger Cars & Diesel 1,4-2,0 I & Conventional & 1846 & 1160 & 463 & 210 \\
\hline Passenger Cars & Diesel $1,4-2,0$ I & PC Euro 1 - 91/441/EEC & 10688 & 8995 & 4922 & 3132 \\
\hline Passenger Cars & Diesel $1,4-2,0$ I & PC Euro 2 - 94/12/EEC & 9555 & 8170 & 4540 & 2928 \\
\hline Passenger Cars & Diesel 1,4 - 2,0 I & PC Euro 3 - 98/69/EC Stage2000 & 28018 & 29524 & 19890 & 15898 \\
\hline Passenger Cars & Diesel $1,4-2,0 \mid$ & PC Euro 4 - 98/69/EC Stage2005 & 4171 & 5484 & 7665 & 9459 \\
\hline Passenger Cars & Diesel $1,4-2,0$ | & PC Euro 5 - EC 715/2007 & 0 & 0 & 3397 & 5768 \\
\hline Passenger Cars & Diesel $>2,0$ | & Conventional & 207 & 90 & 24 & 6 \\
\hline Passenger Cars & Diesel $>2,0$ I & PC Euro 1 - 91/441/EEC & 2806 & 2147 & 1119 & 653 \\
\hline Passenger Cars & Diesel $>2,0 \mathrm{l}$ & PC Euro 2 - 94/12/EEC & 2339 & 1838 & 978 & 577 \\
\hline Passenger Cars & Diesel $>2,0 \mathrm{l}$ & PC Euro 3 - 98/69/EC Stage2000 & 10394 & 10818 & 7707 & 6529 \\
\hline Passenger Cars & Diesel $>2,0 \mathrm{I}$ & PC Euro 4 - 98/69/EC Stage2005 & 2015 & 2560 & 2757 & 3115 \\
\hline Passenger Cars & Diesel $>2,0$ I & PC Euro 5 - EC 715/2007 & 0 & 0 & 791 & 1357 \\
\hline Passenger Cars & LPG & Conventional & 2 & 0 & 0 & 0 \\
\hline Passenger Cars & LPG & PC Euro 1 - 91/441/EEC & 35 & 9 & 1 & 0 \\
\hline Passenger Cars & LPG & PC Euro 2 - 94/12/EEC & 594 & 395 & 165 & 68 \\
\hline Passenger Cars & LPG & PC Euro 3 - 98/69/EC Stage2000 & 2373 & 2421 & 1664 & 1337 \\
\hline Passenger Cars & LPG & 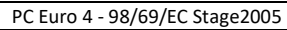 & 441 & 561 & 598 & 676 \\
\hline Passenger Cars & LPG & PC Euro 5 - EC 715/2007 & 0 & 0 & 168 & 293 \\
\hline Passenger Cars & 2-Stroke & Conventional & 0 & 0 & 33056 & 44392 \\
\hline Passenger Cars & Hybrid Gasoline 1,4-2,0I & PC Euro 4 - 98/69/EC Stage2005 & 0 & 0 & 37732 & 50800 \\
\hline Heavy Duty Trucks & Rigid $<=7,5 \mathrm{t}$ & Conventional & 124283 & 97591 & 90921 & 86009 \\
\hline Heavy Duty Trucks & Rigid $<=7,5 \mathrm{t}$ & HD Euro I - 91/542/EEC Stage I & 55095 & 43305 & 40425 & 38769 \\
\hline Heavy Duty Trucks & Rigid $<=7,5 \mathrm{t}$ & HD Euro II - 91/542/EEC Stage II & 90464 & 71103 & 66370 & 63654 \\
\hline Heavy Duty Trucks & Rigid $<=7,5 \mathrm{t}$ & HD Euro III - 2000 Standards & 102819 & 80812 & 75437 & 72347 \\
\hline Heavy Duty Trucks & Rigid $<=7,5 \mathrm{t}$ & HD Euro IV - 2005 Standards & 42096 & 45027 & 42738 & 49247 \\
\hline Heavy Duty Trucks & Rigid $<=7,5 \mathrm{t}$ & HD Euro V - 2008 Standards & 0 & 10421 & 10372 & 18212 \\
\hline Heavy Duty Trucks & Rigid $<=7,5 \mathrm{t}$ & HD Euro VI & 0 & 0 & 0 & 0 \\
\hline Heavy Duty Trucks & Rigid $7,5-12 \mathrm{t}$ & Conventional & 55912 & 43903 & 40902 & 38696 \\
\hline Heavy Duty Trucks & Rigid $7,5-12 \mathrm{t}$ & HD Euro I - 91/542/EEC Stage I & 24788 & 19481 & 18187 & 17442 \\
\hline Heavy Duty Trucks & Rigid $7,5-12 \mathrm{t}$ & HD Euro II - 91/542/EEC Stage II & 40697 & 31989 & 29861 & 28637 \\
\hline Heavy Duty Trucks & Rigid $7,5-12 \mathrm{t}$ & HD Euro III - 2000 Standards & 46256 & 36357 & 33940 & 32550 \\
\hline Heavy Duty Trucks & Rigid $7,5-12 t$ & HD Euro IV - 2005 Standards & 18938 & 20257 & 19227 & 22157 \\
\hline Heavy Duty Trucks & Rigid $7,5-12 \mathrm{t}$ & HD Euro V - 2008 Standards & 0 & 4691 & 4666 & 8193 \\
\hline Heavy Duty Trucks & Rigid $7,5-12 \mathrm{t}$ & HD Euro VI & 0 & 0 & 0 & 0 \\
\hline Heavy Duty Trucks & Rigid $12-14 \mathrm{t}$ & Conventional & 15644 & 12283 & 11445 & 10825 \\
\hline Heavy Duty Trucks & Rigid $12-14 \mathrm{t}$ & HD Euro I - 91/542/EEC Stage I & 6933 & 5450 & 5086 & 4880 \\
\hline Heavy Duty Trucks & Rigid $12-14 t$ & HD Euro II - 91/542/EEC Stage II & 11384 & 8949 & 8353 & 8012 \\
\hline Heavy Duty Trucks & Rigid $12-14 t$ & HD Euro III - 2000 Standards & 12940 & 10171 & 9495 & 9104 \\
\hline Heavy Duty Trucks & Rigid $12-14 t$ & HD Euro IV - 2005 Standards & 5298 & 5667 & 5380 & 6198 \\
\hline Heavy Duty Trucks & Rigid $12-14 t$ & HD Euro V - 2008 Standards & 0 & 1311 & 1305 & 2293 \\
\hline Heavy Duty Trucks & Rigid $12-14 \mathrm{t}$ & HD Euro VI & 0 & 0 & 0 & 0 \\
\hline Heavy Duty Trucks & Rigid $14-20 t$ & Conventional & 43085 & 33834 & 31521 & 29818 \\
\hline Heavy Duty Trucks & Rigid $14-20 t$ & HD Euro I - 91/542/EEC Stage I & 19100 & 15015 & 14015 & 13440 \\
\hline Heavy Duty Trucks & Rigid $14-20 t$ & HD Euro II - 91/542/EEC Stage II & 31361 & 24651 & 23009 & 22068 \\
\hline Heavy Duty Trucks & Rigid $14-20 t$ & HD Euro III - 2000 Standards & 35645 & 28019 & 26153 & 25083 \\
\hline Heavy Duty Trucks & Rigid $14-20 t$ & HD Euro IV - 2005 Standards & 14595 & 15608 & 14814 & 17071 \\
\hline Heavy Duty Trucks & Rigid $14-20 t$ & HD Euro V - 2008 Standards & 0 & 3614 & 3598 & 6315 \\
\hline Heavy Duty Trucks & Rigid $14-20 t$ & HD Euro VI & 0 & 0 & 0 & 0 \\
\hline Heavy Duty Trucks & Rigid $20-26 \mathrm{t}$ & Conventional & 35020 & 27497 & 25619 & 24236 \\
\hline Heavy Duty Trucks & Rigid $20-26 \mathrm{t}$ & HD Euro I - 91/542/EEC Stage I & 15525 & 12203 & 11388 & 10925 \\
\hline Heavy Duty Trucks & Rigid $20-26 \mathrm{t}$ & HD Euro II - 91/542/EEC Stage II & 25487 & 20032 & 18701 & 17938 \\
\hline Heavy Duty Trucks & Rigid $20-26 \mathrm{t}$ & HD Euro III - 2000 Standards & 28968 & 22772 & 21255 & 20384 \\
\hline Heavy Duty Trucks & Rigid $20-26 \mathrm{t}$ & HD Euro IV - 2005 Standards & 11862 & 12686 & 12041 & 13875 \\
\hline Heavy Duty Trucks & Rigid $20-26 \mathrm{t}$ & HD Euro V - 2008 Standards & 0 & 2935 & 2925 & 5130 \\
\hline Heavy Duty Trucks & Rigid $20-26 \mathrm{t}$ & HD Euro VI & 0 & 0 & 0 & 0 \\
\hline Heavy Duty Trucks & Rigid $26-28 \mathrm{t}$ & Conventional & 89 & 72 & 65 & 64 \\
\hline Heavy Duty Trucks & Rigid $26-28 t$ & HD Euro I - 91/542/EEC Stage I & 39 & 30 & 29 & 28 \\
\hline Heavy Duty Trucks & Rigid $26-28 \mathrm{t}$ & HD Euro II - 91/542/EEC Stage II & 64 & 51 & 49 & 48 \\
\hline Heavy Duty Trucks & Rigid $26-28 t$ & HD Euro III - 2000 Standards & 74 & 59 & 53 & 52 \\
\hline Heavy Duty Trucks & Rigid $26-28 \mathrm{t}$ & HD Euro IV - 2005 Standards & 30 & 34 & 33 & 36 \\
\hline Heavy Duty Trucks & Rigid $26-28 t$ & HD Euro V - 2008 Standards & 0 & 8 & 8 & 12 \\
\hline Heavy Duty Trucks & Rigid $26-28 \mathrm{t}$ & HD Euro VI & 0 & 0 & 0 & 0 \\
\hline Heavy Duty Trucks & Rigid $28-32 t$ & Conventional & 3279 & 2575 & 2398 & 2269 \\
\hline Heavy Duty Trucks & Rigid $28-32 t$ & HD Euro I - 91/542/EEC Stage I & 1453 & 1141 & 1065 & 1024 \\
\hline Heavy Duty Trucks & Rigid $28-32 t$ & HD Euro II - 91/542/EEC Stage II & 2388 & 1875 & 1750 & 1681 \\
\hline Heavy Duty Trucks & Rigid $28-32 \mathrm{t}$ & HD Euro III - 2000 Standards & 2713 & 2133 & 1990 & 1910 \\
\hline Heavy Duty Trucks & Rigid $28-32 \mathrm{t}$ & HD Euro IV - 2005 Standards & 1113 & 1188 & 1126 & 1298 \\
\hline
\end{tabular}




\begin{tabular}{|c|c|c|c|c|c|c|c|c|c|}
\hline Sector & Subsector & Technology & 2008 & 2009 & 2010 & 2011 & 2012 & 2013 & 2014 \\
\hline Heavy Duty Trucks & Rigid $28-32 \mathrm{t}$ & HD Euro V - 2008 Standards & 0 & 276 & 273 & 480 & 580 & 661 & 841 \\
\hline Heavy Duty Trucks & Rigid $28-32 \mathrm{t}$ & HD Euro VI & 0 & 0 & 0 & 0 & 0 & 0 & 208 \\
\hline Heavy Duty Trucks & Rigid $>32 t$ & Conventional & 18086 & 14205 & 13232 & 12517 & 9585 & 7684 & 7609 \\
\hline Heavy Duty Trucks & Rigid $>32 t$ & HD Euro I - 91/542/EEC Stage I & 8021 & 6303 & 5882 & 5642 & 4452 & 3680 & 3806 \\
\hline Heavy Duty Trucks & Rigid $>32 t$ & HD Euro II - 91/542/EEC Stage II & 13167 & 10349 & 9659 & 9265 & 7308 & 6043 & 6248 \\
\hline Heavy Duty Trucks & Rigid $>32 t$ & HD Euro III - 2000 Standards & 14964 & 11761 & 10980 & 10530 & 8307 & 6869 & 7101 \\
\hline Heavy Duty Trucks & Rigid $>32 \mathrm{t}$ & HD Euro IV - 2005 Standards & 6125 & 6553 & 6220 & 7169 & 6669 & 6345 & 6557 \\
\hline Heavy Duty Trucks & Rigid $>32 t$ & HD Euro V - 2008 Standards & 0 & 1518 & 1509 & 2652 & 3204 & 3641 & 4644 \\
\hline Heavy Duty Trucks & Rigid $>32 t$ & HD Euro VI & 0 & 0 & 0 & 0 & 0 & 0 & 1156 \\
\hline Heavy Duty Trucks & Articulated $14-20 \mathrm{t}$ & Conventional & 7194 & 5594 & 5245 & 5042 & 3901 & 3164 & 3182 \\
\hline Heavy Duty Trucks & Articulated $14-20 t$ & HD Euro I - 91/542/EEC Stage I & 1251 & 976 & 918 & 883 & 701 & 580 & 603 \\
\hline Heavy Duty Trucks & Articulated $14-20 \mathrm{t}$ & HD Euro II - 91/542/EEC Stage II & 1704 & 1336 & 1256 & 1213 & 960 & 798 & 829 \\
\hline Heavy Duty Trucks & Articulated $14-20 \mathrm{t}$ & HD Euro III - 2000 Standards & 2733 & 2155 & 2031 & 1967 & 1563 & 1303 & 1358 \\
\hline Heavy Duty Trucks & Articulated $14-20 \mathrm{t}$ & HD Euro IV - 2005 Standards & 1581 & 1692 & 1578 & 1769 & 1635 & 1548 & 1616 \\
\hline Heavy Duty Trucks & Articulated $14-20 t$ & HD Euro V - 2008 Standards & 0 & 390 & 351 & 580 & 708 & 812 & 1046 \\
\hline Heavy Duty Trucks & Articulated $14-20 \mathrm{t}$ & HD Euro VI & 0 & 0 & 0 & 0 & 0 & 0 & 258 \\
\hline Heavy Duty Trucks & Articulated $20-28 \mathrm{t}$ & Conventional & 4717 & 3665 & 3438 & 3305 & 2558 & 2073 & 2086 \\
\hline Heavy Duty Trucks & Articulated $20-28 \mathrm{t}$ & HD Euro I - 91/542/EEC Stage I & 817 & 640 & 600 & 580 & 459 & 379 & 395 \\
\hline Heavy Duty Trucks & Articulated $20-28 \mathrm{t}$ & HD Euro II - 91/542/EEC Stage II & 1118 & 874 & 824 & 794 & 629 & 525 & 544 \\
\hline Heavy Duty Trucks & Articulated $20-28 \mathrm{t}$ & HD Euro III - 2000 Standards & 1792 & 1412 & 1330 & 1290 & 1025 & 854 & 888 \\
\hline Heavy Duty Trucks & Articulated $20-28 \mathrm{t}$ & HD Euro IV - 2005 Standards & 1034 & 1111 & 1036 & 1157 & 1071 & 1016 & 1058 \\
\hline Heavy Duty Trucks & Articulated $20-28 \mathrm{t}$ & HD Euro V - 2008 Standards & 0 & 254 & 232 & 379 & 465 & 533 & 686 \\
\hline Heavy Duty Trucks & Articulated $20-28 \mathrm{t}$ & HD Euro VI & 0 & 0 & 0 & 0 & 0 & 0 & 169 \\
\hline Heavy Duty Trucks & Articulated $28-34 \mathrm{t}$ & Conventional & 3723 & 2893 & 2712 & 2607 & 2018 & 1638 & 1646 \\
\hline Heavy Duty Trucks & Articulated $28-34 \mathrm{t}$ & HD Euro I - 91/542/EEC Stage I & 645 & 505 & 473 & 455 & 360 & 301 & 312 \\
\hline Heavy Duty Trucks & Articulated $28-34 \mathrm{t}$ & HD Euro II - 91/542/EEC Stage II & 881 & 691 & 649 & 625 & 498 & 413 & 428 \\
\hline Heavy Duty Trucks & Articulated $28-34 \mathrm{t}$ & HD Euro III - 2000 Standards & 1413 & 1115 & 1048 & 1016 & 809 & 675 & 701 \\
\hline Heavy Duty Trucks & Articulated $28-34 \mathrm{t}$ & HD Euro IV - 2005 Standards & 817 & 874 & 816 & 915 & 845 & 801 & 835 \\
\hline Heavy Duty Trucks & Articulated $28-34 \mathrm{t}$ & HD Euro V - 2008 Standards & 0 & 204 & 184 & 298 & 367 & 421 & 541 \\
\hline Heavy Duty Trucks & Articulated $28-34 \mathrm{t}$ & HD Euro VI & 0 & 0 & 0 & 0 & 0 & 0 & 134 \\
\hline Heavy Duty Trucks & Articulated $34-40 \mathrm{t}$ & Conventional & 56725 & 44107 & 41347 & 39740 & 30775 & 24957 & 25081 \\
\hline Heavy Duty Trucks & Articulated $34-40 t$ & HD Euro I - 91/542/EEC Stage I & 9848 & 7707 & 7228 & 6964 & 5520 & 4581 & 4754 \\
\hline Heavy Duty Trucks & Articulated $34-40 \mathrm{t}$ & HD Euro II - 91/542/EEC Stage II & 13443 & 10536 & 9899 & 9551 & 7577 & 6297 & 6539 \\
\hline Heavy Duty Trucks & Articulated $34-40 \mathrm{t}$ & HD Euro III - 2000 Standards & 21567 & 16978 & 16009 & 15499 & 12333 & 10278 & 10702 \\
\hline Heavy Duty Trucks & Articulated $34-40 \mathrm{t}$ & HD Euro IV - 2005 Standards & 12453 & 13348 & 12449 & 13940 & 12883 & 12206 & 12737 \\
\hline Heavy Duty Trucks & Articulated $34-40 \mathrm{t}$ & HD Euro V - 2008 Standards & 0 & 3084 & 2774 & 4566 & 5588 & 6409 & 8256 \\
\hline Heavy Duty Trucks & Articulated $34-40 \mathrm{t}$ & HD Euro VI & 0 & 0 & 0 & 0 & 0 & 0 & 2044 \\
\hline Heavy Duty Trucks & Articulated $40-50 \mathrm{t}$ & Conventional & 266 & 204 & 192 & 185 & 144 & 117 & 116 \\
\hline Heavy Duty Trucks & Articulated $40-50 \mathrm{t}$ & HD Euro I - 91/542/EEC Stage I & 44 & 34 & 33 & 32 & 26 & 22 & 21 \\
\hline Heavy Duty Trucks & Articulated $40-50 \mathrm{t}$ & HD Euro II - 91/542/EEC Stage II & 64 & 51 & 45 & 44 & 36 & 31 & 30 \\
\hline Heavy Duty Trucks & Articulated $40-50 \mathrm{t}$ & HD Euro III - 2000 Standards & 98 & 81 & 73 & 73 & 59 & 47 & 51 \\
\hline Heavy Duty Trucks & Articulated $40-50 \mathrm{t}$ & HD Euro IV - 2005 Standards & 59 & 64 & 57 & 64 & 59 & 56 & 59 \\
\hline Heavy Duty Trucks & Articulated $40-50 \mathrm{t}$ & HD Euro V - 2008 Standards & 0 & 13 & 12 & 20 & 26 & 31 & 39 \\
\hline Heavy Duty Trucks & Articulated $40-50 \mathrm{t}$ & HD Euro VI & 0 & 0 & 0 & 0 & 0 & 0 & 9 \\
\hline Heavy Duty Trucks & Articulated $50-60 \mathrm{t}$ & Conventional & 108 & 85 & 82 & 77 & 59 & 47 & 48 \\
\hline Heavy Duty Trucks & Articulated $50-60 \mathrm{t}$ & HD Euro I - 91/542/EEC Stage I & 20 & 17 & 12 & 12 & 10 & 8 & 9 \\
\hline Heavy Duty Trucks & Articulated $50-60 \mathrm{t}$ & HD Euro II - 91/542/EEC Stage II & 25 & 21 & 20 & 20 & 13 & 11 & 12 \\
\hline Heavy Duty Trucks & Articulated $50-60 \mathrm{t}$ & HD Euro III - 2000 Standards & 39 & 34 & 33 & 28 & 23 & 20 & 21 \\
\hline Heavy Duty Trucks & Articulated $50-60 \mathrm{t}$ & HD Euro IV - 2005 Standards & 25 & 25 & 24 & 28 & 26 & 22 & 24 \\
\hline Heavy Duty Trucks & Articulated $50-60 \mathrm{t}$ & HD Euro V - 2008 Standards & 0 & 4 & 4 & 8 & 10 & 11 & 15 \\
\hline Heavy Duty Trucks & Articulated $50-60 \mathrm{t}$ & HD Euro VI & 0 & 0 & 0 & 0 & 0 & 0 & 3 \\
\hline
\end{tabular}




\section{Pirgetos Station}

\begin{tabular}{|c|c|c|c|c|c|c|}
\hline Sector & Subsector & Technology & 2008 & 2009 & 2010 & 2011 \\
\hline Passenger Cars & Gasoline $0,8-1,4$ I & ECE 15/02 & 11300 & 5301 & 1041 & 0 \\
\hline Passenger Cars & Gasoline $0,8-1,4$ I & ECE $15 / 03$ & 104401 & 80053 & 34358 & 23808 \\
\hline Passenger Cars & Gasoline $0,8-1,4 \mid$ & ECE 15/04 & 645180 & 536132 & 246572 & 180134 \\
\hline Passenger Cars & Gasoline $0,8-1,4$ I & PC Euro 1 - 91/441/EEC & 709625 & 621449 & 302064 & 233703 \\
\hline Passenger Cars & Gasoline $0,8-1,4$ I & PC Euro 2 - 94/12/EEC & 1147031 & 1037140 & 523281 & 422708 \\
\hline Passenger Cars & Gasoline $0,8-1,4$ I & PC Euro 3 - 98/69/EC Stage2000 & 1201405 & 1168376 & 599645 & 494595 \\
\hline Passenger Cars & Gasoline $0,8-1,4$ I & $\begin{array}{l}\text { PC Euro } 4 \text { - 98/69/EC Stage2005 } \\
\end{array}$ & 235204 & 275166 & 206444 & 211427 \\
\hline Passenger Cars & Gasoline $0,8-1,4$ I & PC Euro 5 - EC 715/2007 & 0 & 0 & 58965 & 88976 \\
\hline Passenger Cars & Gasoline $1,4-2,0$ I & ECE $15 / 02$ & 1631 & 681 & 123 & 0 \\
\hline Passenger Cars & Gasoline $1,4-2,0$ I & ECE $15 / 03$ & 23619 & 17362 & 7432 & 5175 \\
\hline Passenger Cars & Gasoline 1,4-2,0I & ECE 15/04 & 168834 & 133277 & 59971 & 42794 \\
\hline Passenger Cars & Gasoline 1,4-2,0 I & PC Euro 1 - 91/441/EEC & 302354 & 256161 & 124204 & 95797 \\
\hline Passenger Cars & Gasoline $1,4-2,0$ I & PC Euro 2 - 94/12/EEC & 469122 & 409966 & 206080 & 165690 \\
\hline Passenger Cars & Gasoline $1,4-2,0 \mathrm{I}$ & PC Euro 3 - 98/69/EC Stage2000 & 774853 & 740141 & 379535 & 312662 \\
\hline Passenger Cars & Gasoline 1,4-2,0 I & PC Euro 4 - 98/69/EC Stage2005 & 176200 & 202765 & 130968 & 124561 \\
\hline Passenger Cars & Gasoline 1,4-2,0 I & PC Euro 5 - EC 715/2007 & 0 & 0 & 24133 & 35894 \\
\hline Passenger Cars & Gasoline $>2,0$ I & ECE 15/02 & 799 & 443 & 115 & 0 \\
\hline Passenger Cars & Gasoline $>2,0 \mathrm{I}$ & ECE 15/03 & 1528 & 1208 & 597 & 475 \\
\hline Passenger Cars & Gasoline $>2,0 \mathrm{I}$ & ECE 15/04 & 3024 & 2409 & 1200 & 962 \\
\hline Passenger Cars & Gasoline $>2,0 \mathrm{I}$ & PC Euro 1 - 91/441/EEC & 8610 & 6890 & 3447 & 2776 \\
\hline Passenger Cars & Gasoline $>2,0 \mathrm{I}$ & PC Euro 2 - 94/12/EEC & 12502 & 10076 & 5077 & 4115 \\
\hline Passenger Cars & Gasoline $>2,0 \mathrm{I}$ & PC Euro 3 - 98/69/EC Stage2000 & 53303 & 49031 & 24917 & 20362 \\
\hline Passenger Cars & Gasoline $>2,0$ I & PC Euro 4 - 98/69/EC Stage2005 & 17415 & 19201 & 10909 & 9552 \\
\hline Passenger Cars & Gasoline $>2,0$ I & PC Euro 5 - EC 715/2007 & 0 & 0 & 1016 & 1438 \\
\hline Passenger Cars & Diesel 1,4-2,0 I & Conventional & 2232 & 1317 & 365 & 152 \\
\hline Passenger Cars & Diesel $1,4-2,0$ I & PC Euro 1 - 91/441/EEC & 12920 & 10209 & 3877 & 2268 \\
\hline Passenger Cars & Diesel $1,4-2,0$ I & PC Euro 2 - 94/12/EEC & 11551 & 9273 & 3576 & 2121 \\
\hline Passenger Cars & Diesel $1,4-2,0 \mid$ & PC Euro 3 - 98/69/EC Stage2000 & 33870 & 33510 & 15667 & 11514 \\
\hline Passenger Cars & Diesel $1,4-2,0 \mid$ & PC Euro 4 - 98/69/EC Stage2005 & 5042 & 6224 & 6037 & 6851 \\
\hline Passenger Cars & Diesel $1,4-2,0 \mid$ & PC Euro 5 - EC 715/2007 & 0 & 0 & 2675 & 4177 \\
\hline Passenger Cars & Diesel $>2,0$ I & Conventional & 250 & 102 & 19 & 4 \\
\hline Passenger Cars & Diesel >2,0 I & PC Euro 1 - 91/441/EEC & 3393 & 2437 & 882 & 473 \\
\hline Passenger Cars & Diesel $>2,0$ I & PC Euro 2 - 94/12/EEC & 2828 & 2086 & 771 & 418 \\
\hline Passenger Cars & Diesel $>2,0 \mathrm{I}$ & PC Euro 3 - 98/69/EC Stage2000 & 12564 & 12278 & 6071 & 4729 \\
\hline Passenger Cars & Diesel $>2,0$ I & $\begin{array}{l}\text { PC Euro } 4 \text { - 98/69/EC Stage2005 } \\
\end{array}$ & 2436 & 2905 & 2172 & 2256 \\
\hline Passenger Cars & Diesel $>2,0$ I & PC Euro 5 - EC 715/2007 & 0 & 0 & 623 & 983 \\
\hline Passenger Cars & LPG & Conventional & 3 & 0 & 0 & 0 \\
\hline Passenger Cars & LPG & PC Euro 1 - 91/441/EEC & 43 & 10 & 1 & 0 \\
\hline Passenger Cars & LPG & PC Euro 2 - 94/12/EEC & 718 & 448 & 130 & 49 \\
\hline Passenger Cars & LPG & PC Euro 3 - 98/69/EC Stage2000 & 2869 & 2747 & 1311 & 968 \\
\hline Passenger Cars & LPG & 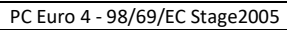 & 533 & 637 & 471 & 490 \\
\hline Passenger Cars & LPG & PC Euro 5 - EC 715/2007 & 0 & 0 & 132 & 212 \\
\hline Passenger Cars & 2-Stroke & Conventional & 0 & 0 & 26037 & 32152 \\
\hline Passenger Cars & Hybrid Gasoline 1,4-2,0 I & PC Euro 4 - 98/69/EC Stage2005 & 0 & 0 & 29720 & 36793 \\
\hline Heavy Duty Trucks & Rigid $<=7,5 \mathrm{t}$ & Conventional & 171730 & 130664 & 63054 & 91852 \\
\hline Heavy Duty Trucks & Rigid $<=7,5 \mathrm{t}$ & HD Euro I - 91/542/EEC Stage I & 76129 & 57981 & 28035 & 41403 \\
\hline Heavy Duty Trucks & Rigid $<=7,5 \mathrm{t}$ & HD Euro II - 91/542/EEC Stage II & 125001 & 95200 & 46029 & 67979 \\
\hline Heavy Duty Trucks & Rigid $<=7,5 \mathrm{t}$ & HD Euro III - 2000 Standards & 142072 & 108199 & 52317 & 77262 \\
\hline Heavy Duty Trucks & Rigid $<=7,5 \mathrm{t}$ & HD Euro IV - 2005 Standards & 58166 & 60286 & 29639 & 52593 \\
\hline Heavy Duty Trucks & Rigid $<=7,5 \mathrm{t}$ & HD Euro V - 2008 Standards & 0 & 13953 & 7193 & 19449 \\
\hline Heavy Duty Trucks & Rigid $<=7,5 \mathrm{t}$ & HD Euro VI & 0 & 0 & 0 & 0 \\
\hline Heavy Duty Trucks & Rigid $7,5-12 \mathrm{t}$ & Conventional & 77258 & 58782 & 28366 & 41325 \\
\hline Heavy Duty Trucks & Rigid $7,5-12 \mathrm{t}$ & HD Euro I - 91/542/EEC Stage I & 34251 & 26083 & 12613 & 18627 \\
\hline Heavy Duty Trucks & Rigid $7,5-12 \mathrm{t}$ & HD Euro II - 91/542/EEC Stage II & 56234 & 42830 & 20709 & 30583 \\
\hline Heavy Duty Trucks & Rigid $7,5-12 \mathrm{t}$ & HD Euro III - 2000 Standards & 63916 & 48679 & 23538 & 34762 \\
\hline Heavy Duty Trucks & Rigid $7,5-12 \mathrm{t}$ & HD Euro IV - 2005 Standards & 26168 & 27122 & 13334 & 23662 \\
\hline Heavy Duty Trucks & Rigid $7,5-12 \mathrm{t}$ & HD Euro V - 2008 Standards & 0 & 6281 & 3236 & 8750 \\
\hline Heavy Duty Trucks & Rigid $7,5-12 \mathrm{t}$ & HD Euro VI & 0 & 0 & 0 & 0 \\
\hline Heavy Duty Trucks & Rigid $12-14 \mathrm{t}$ & Conventional & 21616 & 16446 & 7937 & 11560 \\
\hline Heavy Duty Trucks & Rigid $12-14 \mathrm{t}$ & HD Euro I - 91/542/EEC Stage I & 9580 & 7297 & 3527 & 5212 \\
\hline Heavy Duty Trucks & Rigid $12-14 t$ & HD Euro II - 91/542/EEC Stage II & 15731 & 11982 & 5793 & 8556 \\
\hline Heavy Duty Trucks & Rigid $12-14 t$ & HD Euro III - 2000 Standards & 17881 & 13618 & 6585 & 9722 \\
\hline Heavy Duty Trucks & Rigid $12-14 t$ & HD Euro IV - 2005 Standards & 7321 & 7587 & 3731 & 6619 \\
\hline Heavy Duty Trucks & Rigid $12-14 \mathrm{t}$ & HD Euro V - 2008 Standards & 0 & 1755 & 905 & 2449 \\
\hline Heavy Duty Trucks & Rigid $12-14 t$ & HD Euro VI & 0 & 0 & 0 & 0 \\
\hline Heavy Duty Trucks & Rigid $14-20 t$ & Conventional & 59534 & 45300 & 21860 & 31844 \\
\hline Heavy Duty Trucks & Rigid $14-20 t$ & HD Euro I - 91/542/EEC Stage I & 26392 & 20103 & 9719 & 14353 \\
\hline Heavy Duty Trucks & Rigid $14-20 t$ & HD Euro II - 91/542/EEC Stage II & 43334 & 33005 & 15957 & 23568 \\
\hline Heavy Duty Trucks & Rigid $14-20 t$ & HD Euro III - 2000 Standards & 49253 & 37514 & 18138 & 26787 \\
\hline Heavy Duty Trucks & Rigid $14-20 t$ & HD Euro IV - 2005 Standards & 20167 & 20898 & 10274 & 18231 \\
\hline Heavy Duty Trucks & Rigid $14-20 t$ & HD Euro V - 2008 Standards & 0 & 4838 & 2495 & 6744 \\
\hline Heavy Duty Trucks & Rigid $14-20 t$ & HD Euro VI & 0 & 0 & 0 & 0 \\
\hline Heavy Duty Trucks & Rigid $20-26 \mathrm{t}$ & Conventional & 48389 & 36816 & 17767 & 25883 \\
\hline Heavy Duty Trucks & Rigid $20-26 \mathrm{t}$ & HD Euro I - 91/542/EEC Stage I & 21453 & 16338 & 7898 & 11668 \\
\hline Heavy Duty Trucks & Rigid $20-26 \mathrm{t}$ & HD Euro II - 91/542/EEC Stage II & 35217 & 26821 & 12970 & 19156 \\
\hline Heavy Duty Trucks & Rigid $20-26 \mathrm{t}$ & HD Euro III - 2000 Standards & 40027 & 30490 & 14740 & 21769 \\
\hline Heavy Duty Trucks & Rigid $20-26 \mathrm{t}$ & HD Euro IV - 2005 Standards & 16391 & 16985 & 8350 & 14818 \\
\hline Heavy Duty Trucks & Rigid $20-26 \mathrm{t}$ & HD Euro V - 2008 Standards & 0 & 3930 & 2028 & 5479 \\
\hline Heavy Duty Trucks & Rigid $20-26 \mathrm{t}$ & HD Euro VI & 0 & 0 & 0 & 0 \\
\hline Heavy Duty Trucks & Rigid $26-28 \mathrm{t}$ & Conventional & 122 & 97 & 45 & 69 \\
\hline Heavy Duty Trucks & Rigid $26-28 \mathrm{t}$ & HD Euro I - 91/542/EEC Stage I & 54 & 40 & 20 & 30 \\
\hline Heavy Duty Trucks & Rigid $26-28 \mathrm{t}$ & HD Euro II - 91/542/EEC Stage II & 88 & 68 & 34 & 52 \\
\hline Heavy Duty Trucks & Rigid $26-28 \mathrm{t}$ & HD Euro III - 2000 Standards & 102 & 80 & 37 & 56 \\
\hline Heavy Duty Trucks & Rigid $26-28 \mathrm{t}$ & HD Euro IV - 2005 Standards & 41 & 45 & 23 & 39 \\
\hline Heavy Duty Trucks & Rigid $26-28 t$ & HD Euro V - 2008 Standards & 0 & 11 & 6 & 13 \\
\hline Heavy Duty Trucks & Rigid $26-28 \mathrm{t}$ & HD Euro VI & 0 & 0 & 0 & 0 \\
\hline Heavy Duty Trucks & Rigid $28-32 \mathrm{t}$ & Conventional & 4531 & 3447 & 1663 & 2423 \\
\hline Heavy Duty Trucks & Rigid $28-32 t$ & HD Euro I - 91/542/EEC Stage I & 2007 & 1528 & 738 & 1093 \\
\hline Heavy Duty Trucks & Rigid $28-32 \mathrm{t}$ & HD Euro II - 91/542/EEC Stage II & 3300 & 2510 & 1214 & 1795 \\
\hline Heavy Duty Trucks & Rigid $28-32 \mathrm{t}$ & HD Euro III - 2000 Standards & 3749 & 2856 & 1380 & 2040 \\
\hline Heavy Duty Trucks & Rigid $28-32 \mathrm{t}$ & HD Euro IV - 2005 Standards & 1538 & 1590 & 781 & 1386 \\
\hline
\end{tabular}




\begin{tabular}{|c|c|c|c|c|c|c|c|c|c|}
\hline Sector & Subsector & Technology & 2008 & 2009 & 2010 & 2011 & 2012 & 2013 & 2014 \\
\hline Heavy Duty Trucks & Rigid $28-32 \mathrm{t}$ & HD Euro V - 2008 Standards & 0 & 369 & 190 & 512 & 450 & 573 & 754 \\
\hline Heavy Duty Trucks & Rigid $28-32 \mathrm{t}$ & HD Euro VI & 0 & 0 & 0 & 0 & 0 & 0 & 186 \\
\hline Heavy Duty Trucks & Rigid $>32 t$ & Conventional & 24991 & 19018 & 9176 & 13368 & 7443 & 6656 & 6820 \\
\hline Heavy Duty Trucks & Rigid $>32 t$ & HD Euro I - 91/542/EEC Stage I & 11084 & 8439 & 4079 & 6025 & 3457 & 3188 & 3411 \\
\hline Heavy Duty Trucks & Rigid $>32 t$ & HD Euro II - 91/542/EEC Stage II & 18194 & 13856 & 6698 & 9894 & 5675 & 5235 & 5600 \\
\hline Heavy Duty Trucks & Rigid $>32 t$ & HD Euro III - 2000 Standards & 20677 & 15747 & 7615 & 11246 & 6451 & 5950 & 6365 \\
\hline Heavy Duty Trucks & Rigid $>32 \mathrm{t}$ & HD Euro IV - 2005 Standards & 8464 & 8774 & 4314 & 7656 & 5179 & 5496 & 5877 \\
\hline Heavy Duty Trucks & Rigid $>32 t$ & HD Euro V - 2008 Standards & 0 & 2033 & 1047 & 2832 & 2488 & 3154 & 4162 \\
\hline Heavy Duty Trucks & Rigid $>32 t$ & HD Euro VI & 0 & 0 & 0 & 0 & 0 & 0 & 1036 \\
\hline Heavy Duty Trucks & Articulated $14-20 \mathrm{t}$ & Conventional & 9940 & 7490 & 3638 & 5384 & 3030 & 2741 & 2852 \\
\hline Heavy Duty Trucks & Articulated $14-20 t$ & HD Euro I - 91/542/EEC Stage I & 1728 & 1306 & 636 & 943 & 544 & 503 & 541 \\
\hline Heavy Duty Trucks & Articulated $14-20 \mathrm{t}$ & HD Euro II - 91/542/EEC Stage II & 2354 & 1789 & 871 & 1295 & 745 & 691 & 743 \\
\hline Heavy Duty Trucks & Articulated $14-20 \mathrm{t}$ & HD Euro III - 2000 Standards & 3776 & 2885 & 1409 & 2100 & 1213 & 1129 & 1217 \\
\hline Heavy Duty Trucks & Articulated $14-20 \mathrm{t}$ & HD Euro IV - 2005 Standards & 2184 & 2266 & 1095 & 1889 & 1269 & 1341 & 1449 \\
\hline Heavy Duty Trucks & Articulated $14-20 t$ & HD Euro V - 2008 Standards & 0 & 522 & 243 & 620 & 549 & 703 & 937 \\
\hline Heavy Duty Trucks & Articulated $14-20 \mathrm{t}$ & HD Euro VI & 0 & 0 & 0 & 0 & 0 & 0 & 232 \\
\hline Heavy Duty Trucks & Articulated $20-28 \mathrm{t}$ & Conventional & 6518 & 4907 & 2385 & 3529 & 1987 & 1796 & 1869 \\
\hline Heavy Duty Trucks & Articulated $20-28 \mathrm{t}$ & HD Euro I - 91/542/EEC Stage I & 1129 & 858 & 416 & 620 & 356 & 329 & 354 \\
\hline Heavy Duty Trucks & Articulated $20-28 \mathrm{t}$ & HD Euro II - 91/542/EEC Stage II & 1544 & 1170 & 571 & 848 & 488 & 454 & 487 \\
\hline Heavy Duty Trucks & Articulated $20-28 \mathrm{t}$ & HD Euro III - 2000 Standards & 2477 & 1891 & 922 & 1377 & 796 & 740 & 796 \\
\hline Heavy Duty Trucks & Articulated $20-28 \mathrm{t}$ & HD Euro IV - 2005 Standards & 1429 & 1488 & 718 & 1235 & 832 & 880 & 948 \\
\hline Heavy Duty Trucks & Articulated $20-28 \mathrm{t}$ & HD Euro V - 2008 Standards & 0 & 341 & 161 & 405 & 361 & 462 & 615 \\
\hline Heavy Duty Trucks & Articulated $20-28 \mathrm{t}$ & HD Euro VI & 0 & 0 & 0 & 0 & 0 & 0 & 152 \\
\hline Heavy Duty Trucks & Articulated $28-34 \mathrm{t}$ & Conventional & 5144 & 3873 & 1881 & 2785 & 1567 & 1419 & 1475 \\
\hline Heavy Duty Trucks & Articulated $28-34 \mathrm{t}$ & HD Euro I - 91/542/EEC Stage I & 891 & 676 & 328 & 486 & 280 & 261 & 280 \\
\hline Heavy Duty Trucks & Articulated $28-34 \mathrm{t}$ & HD Euro II - 91/542/EEC Stage II & 1218 & 926 & 450 & 667 & 387 & 358 & 383 \\
\hline Heavy Duty Trucks & Articulated $28-34 \mathrm{t}$ & HD Euro III - 2000 Standards & 1953 & 1494 & 727 & 1085 & 628 & 585 & 628 \\
\hline Heavy Duty Trucks & Articulated $28-34 \mathrm{t}$ & HD Euro IV - 2005 Standards & 1129 & 1170 & 566 & 977 & 656 & 694 & 748 \\
\hline Heavy Duty Trucks & Articulated $28-34 \mathrm{t}$ & HD Euro V - 2008 Standards & 0 & 273 & 127 & 318 & 285 & 365 & 485 \\
\hline Heavy Duty Trucks & Articulated $28-34 \mathrm{t}$ & HD Euro VI & 0 & 0 & 0 & 0 & 0 & 0 & 120 \\
\hline Heavy Duty Trucks & Articulated $34-40 \mathrm{t}$ & Conventional & 78381 & 59054 & 28674 & 42440 & 23899 & 21618 & 22481 \\
\hline Heavy Duty Trucks & Articulated $34-40 t$ & HD Euro I - 91/542/EEC Stage I & 13608 & 10318 & 5012 & 7437 & 4286 & 3968 & 4261 \\
\hline Heavy Duty Trucks & Articulated $34-40 \mathrm{t}$ & HD Euro II - 91/542/EEC Stage II & 18575 & 14106 & 6865 & 10200 & 5884 & 5455 & 5861 \\
\hline Heavy Duty Trucks & Articulated $34-40 \mathrm{t}$ & HD Euro III - 2000 Standards & 29801 & 22732 & 11103 & 16552 & 9578 & 8903 & 9592 \\
\hline Heavy Duty Trucks & Articulated $34-40 \mathrm{t}$ & HD Euro IV - 2005 Standards & 17207 & 17871 & 8633 & 14887 & 10005 & 10573 & 11417 \\
\hline Heavy Duty Trucks & Articulated $34-40 \mathrm{t}$ & HD Euro V - 2008 Standards & 0 & 4129 & 1924 & 4876 & 4340 & 5551 & 7401 \\
\hline Heavy Duty Trucks & Articulated $34-40 \mathrm{t}$ & HD Euro VI & 0 & 0 & 0 & 0 & 0 & 0 & 1832 \\
\hline Heavy Duty Trucks & Articulated $40-50 \mathrm{t}$ & Conventional & 367 & 273 & 133 & 198 & 112 & 102 & 104 \\
\hline Heavy Duty Trucks & Articulated $40-50 \mathrm{t}$ & HD Euro I - 91/542/EEC Stage I & 61 & 45 & 23 & 34 & 20 & 19 & 19 \\
\hline Heavy Duty Trucks & Articulated $40-50 \mathrm{t}$ & HD Euro II - 91/542/EEC Stage II & 88 & 68 & 31 & 47 & 28 & 27 & 27 \\
\hline Heavy Duty Trucks & Articulated $40-50 \mathrm{t}$ & HD Euro III - 2000 Standards & 136 & 108 & 51 & 77 & 46 & 41 & 45 \\
\hline Heavy Duty Trucks & Articulated $40-50 \mathrm{t}$ & HD Euro IV - 2005 Standards & 82 & 85 & 40 & 69 & 46 & 48 & 53 \\
\hline Heavy Duty Trucks & Articulated $40-50 \mathrm{t}$ & HD Euro V - 2008 Standards & 0 & 17 & 8 & 22 & 20 & 27 & 35 \\
\hline Heavy Duty Trucks & Articulated $40-50 \mathrm{t}$ & HD Euro VI & 0 & 0 & 0 & 0 & 0 & 0 & 8 \\
\hline Heavy Duty Trucks & Articulated $50-60 \mathrm{t}$ & Conventional & 150 & 114 & 57 & 82 & 46 & 41 & 43 \\
\hline Heavy Duty Trucks & Articulated $50-60 \mathrm{t}$ & HD Euro I - 91/542/EEC Stage I & 27 & 23 & 8 & 13 & 8 & 7 & 8 \\
\hline Heavy Duty Trucks & Articulated $50-60 \mathrm{t}$ & HD Euro II - 91/542/EEC Stage II & 34 & 28 & 14 & 22 & 10 & 10 & 11 \\
\hline Heavy Duty Trucks & Articulated $50-60 \mathrm{t}$ & HD Euro III - 2000 Standards & 54 & 45 & 23 & 30 & 18 & 17 & 19 \\
\hline Heavy Duty Trucks & Articulated $50-60 \mathrm{t}$ & HD Euro IV - 2005 Standards & 34 & 34 & 17 & 30 & 20 & 19 & 21 \\
\hline Heavy Duty Trucks & Articulated $50-60 \mathrm{t}$ & HD Euro V - 2008 Standards & 0 & 6 & 3 & 9 & 8 & 10 & 13 \\
\hline Heavy Duty Trucks & Articulated $50-60 \mathrm{t}$ & HD Euro VI & 0 & 0 & 0 & 0 & 0 & 0 & 3 \\
\hline
\end{tabular}


Leptokaria Station

\begin{tabular}{|c|c|c|c|c|c|c|}
\hline Sector & Subsector & Technology & 2008 & 2009 & 2010 & 2011 \\
\hline Passenger Cars & Gasoline 0,8 - 1,4 I & ECE 15/02 & 7800 & 3841 & 898 & 0 \\
\hline Passenger Cars & Gasoline $0,8-1,41$ & ECE 15/03 & 72071 & 58003 & 29629 & 23818 \\
\hline Passenger Cars & Gasoline $0,8-1,4$ I & ECE 15/04 & 445387 & 388460 & 212635 & 180213 \\
\hline Passenger Cars & Gasoline $0,8-1,4$ I & PC Euro 1 - 91/441/EEC & 489875 & 450277 & 260490 & 233806 \\
\hline Passenger Cars & Gasoline $0,8-1,4$ I & PC Euro 2 - 94/12/EEC & 791830 & 751470 & 451260 & 422894 \\
\hline Passenger Cars & Gasoline $0,8-1,4$ I & PC Euro 3 - 98/69/EC Stage2000 & 829366 & 846558 & 517113 & 494813 \\
\hline Passenger Cars & Gasoline $0,8-1,4$ I & PC Euro 4 - 98/69/EC Stage2005 & 162369 & 199374 & 178031 & 211520 \\
\hline Passenger Cars & Gasoline $0,8-1,4 \mathrm{I}$ & PC Euro 5 - EC 715/2007 & 0 & 0 & 50849 & 89015 \\
\hline Passenger Cars & Gasoline 1,4-2,0 I & ECE 15/02 & 1126 & 494 & 106 & 0 \\
\hline Passenger Cars & Gasoline 1,4-2,0 I & ECE 15/03 & 16305 & 12580 & 6409 & 5177 \\
\hline Passenger Cars & Gasoline $1,4-2,0$ I & ECE $15 / 04$ & 116551 & 96567 & 51717 & 42813 \\
\hline Passenger Cars & Gasoline 1,4-2,01 & PC Euro 1 - 91/441/EEC & 208724 & 185604 & 107109 & 95840 \\
\hline Passenger Cars & Gasoline 1,4-2,0 I & PC Euro 2 - 94/12/EEC & 323849 & 297045 & 177717 & 165763 \\
\hline Passenger Cars & Gasoline 1,4-2,0 I & PC Euro 3 - 98/69/EC Stage2000 & 534904 & 536276 & 327299 & 312799 \\
\hline Passenger Cars & Gasoline 1,4-2,0 I & PC Euro 4 - 98/69/EC Stage2005 & 121636 & 146916 & 112943 & 124616 \\
\hline Passenger Cars & Gasoline 1,4-2,01 & PC Euro 5 - EC 715/2007 & 0 & 0 & 20811 & 35909 \\
\hline Passenger Cars & Gasoline $>2,0$ I & ECE $15 / 02$ & 552 & 321 & 99 & 0 \\
\hline Passenger Cars & Gasoline $>2,0 \mathrm{I}$ & ECE 15/03 & 1055 & 875 & 515 & 476 \\
\hline Passenger Cars & Gasoline $>2,0 \mathrm{I}$ & ECE 15/04 & 2088 & 1745 & 1034 & 962 \\
\hline Passenger Cars & Gasoline $>2,0 \mathrm{I}$ & PC Euro 1 - 91/441/EEC & 5943 & 4992 & 2973 & 2777 \\
\hline Passenger Cars & Gasoline $>2,01$ & PC Euro 2 - 94/12/EEC & 8630 & 7300 & 4378 & 4117 \\
\hline Passenger Cars & Gasoline $>2,0$ I & PC Euro 3 - 98/69/EC Stage2000 & 36797 & 35526 & 21488 & 20371 \\
\hline Passenger Cars & Gasoline $>2,0$ I & $\begin{array}{l}\text { PC Euro } 4 \text { - 98/69/EC Stage2005 } \\
\end{array}$ & 12022 & 13912 & 9407 & 9557 \\
\hline Passenger Cars & Gasoline $>2,0 \mathrm{I}$ & PC Euro 5 - EC 715/2007 & 0 & 0 & 876 & 1439 \\
\hline Passenger Cars & Diesel $1,4-2,0$ I & Conventional & 1541 & 954 & 315 & 152 \\
\hline Passenger Cars & Diesel $1,4-2,0$ I & PC Euro 1 - 91/441/EEC & 8919 & 7397 & 3344 & 2269 \\
\hline Passenger Cars & Diesel $1,4-2,0$ I & PC Euro 2 - 94/12/EEC & 7974 & 6719 & 3084 & 2121 \\
\hline Passenger Cars & Diesel 1,4 - 2,0 I & PC Euro 3 - 98/69/EC Stage2000 & 23381 & 24280 & 13510 & 11520 \\
\hline Passenger Cars & Diesel $1,4-2,0$ I & PC Euro 4 - 98/69/EC Stage2005 & 3481 & 4510 & 5206 & 6854 \\
\hline Passenger Cars & Diesel 1,4 - 2,0 I & PC Euro 5 - EC 715/2007 & 0 & 0 & 2307 & 4179 \\
\hline Passenger Cars & Diesel >2,0 I & Conventional & 173 & 74 & 16 & 4 \\
\hline Passenger Cars & Diesel $>2,0 \mathrm{I}$ & PC Euro 1 - 91/441/EEC & 2342 & 1766 & 760 & 473 \\
\hline Passenger Cars & Diesel $>2,0 \mathrm{I}$ & PC Euro 2 - 94/12/EEC & 1952 & 1511 & 665 & 418 \\
\hline Passenger Cars & Diesel $>2,0 \mathrm{I}$ & PC Euro 3 - 98/69/EC Stage2000 & 8674 & 8896 & 5235 & 4731 \\
\hline Passenger Cars & Diesel $>2,0$ I & PC Euro 4 - 98/69/EC Stage2005 & 1681 & 2105 & 1873 & 2257 \\
\hline Passenger Cars & Diesel $>2,0$ I & PC Euro 5 - EC 715/2007 & 0 & 0 & 537 & 983 \\
\hline Passenger Cars & LPG & Conventional & 2 & 0 & 0 & 0 \\
\hline Passenger Cars & LPG & PC Euro 1 - 91/441/EEC & 29 & 7 & 1 & 0 \\
\hline Passenger Cars & LPG & PC Euro 2 - 94/12/EEC & 495 & 325 & 112 & 49 \\
\hline Passenger Cars & LPG & PC Euro 3 - 98/69/EC Stage2000 & 1981 & 1991 & 1130 & 969 \\
\hline Passenger Cars & LPG & PC Euro 4 - 98/69/EC Stage2005 & 368 & 461 & 406 & 490 \\
\hline Passenger Cars & LPG & PC Euro 5 - EC 715/2007 & 0 & 0 & 114 & 212 \\
\hline Passenger Cars & 2-Stroke & Conventional & 0 & 0 & 22453 & 32166 \\
\hline Passenger Cars & Hybrid Gasoline $1,4-2,0$ I & PC Euro 4 - 98/69/EC Stage2005 & 0 & 0 & 25630 & 36809 \\
\hline Heavy Duty Trucks & Rigid $<=7,5 \mathrm{t}$ & Conventional & 131163 & 89052 & 63872 & 91804 \\
\hline Heavy Duty Trucks & Rigid $<=7,5 \mathrm{t}$ & HD Euro I - 91/542/EEC Stage I & 58145 & 39516 & 28399 & 41381 \\
\hline Heavy Duty Trucks & Rigid $<=7,5 \mathrm{t}$ & HD Euro II - 91/542/EEC Stage II & 95473 & 64882 & 46626 & 67943 \\
\hline Heavy Duty Trucks & Rigid $<=7,5 \mathrm{t}$ & HD Euro III - 2000 Standards & 108511 & 73741 & 52995 & 77222 \\
\hline Heavy Duty Trucks & Rigid $<=7,5 \mathrm{t}$ & HD Euro IV - 2005 Standards & 44426 & 41087 & 30024 & 52565 \\
\hline Heavy Duty Trucks & Rigid $<=7,5 \mathrm{t}$ & HD Euro V - 2008 Standards & 0 & 9509 & 7287 & 19439 \\
\hline Heavy Duty Trucks & Rigid $<=7,5 \mathrm{t}$ & HD Euro VI & 0 & 0 & 0 & 0 \\
\hline Heavy Duty Trucks & Rigid 7,5-12t & Conventional & 59008 & 40062 & 28734 & 41304 \\
\hline Heavy Duty Trucks & Rigid 7,5 - $12 \mathrm{t}$ & HD Euro I - 91/542/EEC Stage I & 26160 & 17776 & 12777 & 18617 \\
\hline Heavy Duty Trucks & Rigid $7,5-12 \mathrm{t}$ & HD Euro II - 91/542/EEC Stage II & 42950 & 29190 & 20978 & 30567 \\
\hline Heavy Duty Trucks & Rigid $7,5-12 \mathrm{t}$ & HD Euro III - 2000 Standards & 48817 & 33176 & 23843 & 34744 \\
\hline Heavy Duty Trucks & Rigid $7,5-12 \mathrm{t}$ & HD Euro IV - 2005 Standards & 19986 & 18485 & 13507 & 23650 \\
\hline Heavy Duty Trucks & Rigid $7,5-12 \mathrm{t}$ & HD Euro V - 2008 Standards & 0 & 4281 & 3278 & 8745 \\
\hline Heavy Duty Trucks & Rigid $7,5-12 \mathrm{t}$ & HD Euro VI & 0 & 0 & 0 & 0 \\
\hline Heavy Duty Trucks & Rigid $12-14 \mathrm{t}$ & Conventional & 16510 & 11208 & 8040 & 11554 \\
\hline Heavy Duty Trucks & Rigid $12-14 \mathrm{t}$ & HD Euro I - 91/542/EEC Stage I & 7317 & 4973 & 3573 & 5209 \\
\hline Heavy Duty Trucks & Rigid $12-14 \mathrm{t}$ & HD Euro II - 91/542/EEC Stage II & 12015 & 8166 & 5868 & 8552 \\
\hline Heavy Duty Trucks & Rigid $12-14 \mathrm{t}$ & HD Euro III - 2000 Standards & 13657 & 9281 & 6671 & 9717 \\
\hline Heavy Duty Trucks & Rigid $12-14 \mathrm{t}$ & HD Euro IV - 2005 Standards & 5592 & 5171 & 3779 & 6616 \\
\hline Heavy Duty Trucks & Rigid $12-14 \mathrm{t}$ & HD Euro V - 2008 Standards & 0 & 1196 & 917 & 2448 \\
\hline Heavy Duty Trucks & Rigid $12-14 \mathrm{t}$ & HD Euro VI & 0 & 0 & 0 & 0 \\
\hline Heavy Duty Trucks & Rigid $14-20 t$ & Conventional & 45471 & 30874 & 22144 & 31827 \\
\hline Heavy Duty Trucks & Rigid $14-20 t$ & HD Euro I - 91/542/EEC Stage I & 20158 & 13701 & 9845 & 14346 \\
\hline Heavy Duty Trucks & Rigid $14-20 t$ & HD Euro II - 91/542/EEC Stage II & 33097 & 22494 & 16164 & 23555 \\
\hline Heavy Duty Trucks & Rigid $14-20 t$ & HD Euro III - 2000 Standards & 37618 & 25567 & 18373 & 26773 \\
\hline Heavy Duty Trucks & Rigid $14-20 t$ & HD Euro IV - 2005 Standards & 15403 & 14243 & 10407 & 18221 \\
\hline Heavy Duty Trucks & Rigid $14-20 t$ & HD Euro V - 2008 Standards & 0 & 3298 & 2527 & 6741 \\
\hline Heavy Duty Trucks & Rigid $14-20 t$ & HD Euro VI & 0 & 0 & 0 & 0 \\
\hline Heavy Duty Trucks & Rigid $20-26 \mathrm{t}$ & $\begin{array}{c}\text { Conventional } \\
\end{array}$ & 36958 & 25091 & 17998 & 25870 \\
\hline Heavy Duty Trucks & Rigid $20-26 \mathrm{t}$ & HD Euro I - 91/542/EEC Stage I & 16385 & 11135 & 8000 & 11662 \\
\hline Heavy Duty Trucks & Rigid $20-26 \mathrm{t}$ & HD Euro II - 91/542/EEC Stage II & 26898 & 18280 & 13138 & 19146 \\
\hline Heavy Duty Trucks & Rigid $20-26 \mathrm{t}$ & HD Euro III - 2000 Standards & 30572 & 20780 & 14932 & 21757 \\
\hline Heavy Duty Trucks & Rigid $20-26 \mathrm{t}$ & HD Euro IV - 2005 Standards & 12519 & 11576 & 8459 & 14810 \\
\hline Heavy Duty Trucks & Rigid $20-26 \mathrm{t}$ & HD Euro V - 2008 Standards & 0 & 2678 & 2054 & 5476 \\
\hline Heavy Duty Trucks & Rigid $20-26 \mathrm{t}$ & HD Euro VI & 0 & 0 & 0 & 0 \\
\hline Heavy Duty Trucks & Rigid $26-28 \mathrm{t}$ & Conventional & 94 & 66 & 46 & 69 \\
\hline Heavy Duty Trucks & Rigid $26-28 \mathrm{t}$ & HD Euro I - 91/542/EEC Stage I & 42 & 27 & 20 & 30 \\
\hline Heavy Duty Trucks & Rigid $26-28 \mathrm{t}$ & HD Euro II - 91/542/EEC Stage II & 68 & 46 & 34 & 52 \\
\hline Heavy Duty Trucks & Rigid $26-28 \mathrm{t}$ & HD Euro III - 2000 Standards & 78 & 54 & 37 & 56 \\
\hline Heavy Duty Trucks & Rigid $26-28 \mathrm{t}$ & HD Euro IV - 2005 Standards & 31 & 31 & 23 & 39 \\
\hline Heavy Duty Trucks & Rigid $26-28 \mathrm{t}$ & HD Euro V - 2008 Standards & 0 & 8 & 6 & 13 \\
\hline Heavy Duty Trucks & Rigid $26-28 \mathrm{t}$ & HD Euro VI & 0 & 0 & 0 & 0 \\
\hline Heavy Duty Trucks & Rigid $28-32 \mathrm{t}$ & Conventional & 3461 & 2349 & 1685 & 2422 \\
\hline Heavy Duty Trucks & Rigid $28-32 \mathrm{t}$ & HD Euro I - 91/542/EEC Stage I & 1533 & 1041 & 748 & 1093 \\
\hline Heavy Duty Trucks & Rigid $28-32 \mathrm{t}$ & HD Euro II - 91/542/EEC Stage II & 2520 & 1711 & 1229 & 1794 \\
\hline Heavy Duty Trucks & Rigid $28-32 \mathrm{t}$ & HD Euro III - 2000 Standards & 2863 & 1947 & 1398 & 2039 \\
\hline Heavy Duty Trucks & Rigid $28-32 \mathrm{t}$ & HD Euro IV - 2005 Standards & 1174 & 1084 & 791 & 1385 \\
\hline
\end{tabular}




\begin{tabular}{|c|c|c|c|c|c|c|c|c|c|}
\hline Sector & Subsector & Technology & 2008 & 2009 & 2010 & 2011 & 2012 & 2013 & 2014 \\
\hline Heavy Duty Trucks & Rigid $28-32 \mathrm{t}$ & HD Euro V - 2008 Standards & 0 & 252 & 192 & 512 & 612 & 915 & 1158 \\
\hline Heavy Duty Trucks & Rigid $28-32 \mathrm{t}$ & HD Euro VI & 0 & 0 & 0 & 0 & 0 & 0 & 286 \\
\hline Heavy Duty Trucks & Rigid $>32 t$ & Conventional & 19087 & 12962 & 9295 & 13361 & 10111 & 10633 & 10476 \\
\hline Heavy Duty Trucks & Rigid $>32 \mathrm{t}$ & HD Euro I - 91/542/EEC Stage I & 8465 & 5751 & 4132 & 6022 & 4696 & 5093 & 5240 \\
\hline Heavy Duty Trucks & Rigid $>32 t$ & HD Euro II - 91/542/EEC Stage II & 13896 & 9444 & 6785 & 9889 & 7709 & 8363 & 8603 \\
\hline Heavy Duty Trucks & Rigid $>32 t$ & HD Euro III - 2000 Standards & 15793 & 10732 & 7714 & 11240 & 8763 & 9506 & 9777 \\
\hline Heavy Duty Trucks & Rigid $>32 t$ & HD Euro IV - 2005 Standards & 6465 & 5980 & 4370 & 7652 & 7035 & 8780 & 9028 \\
\hline Heavy Duty Trucks & Rigid $>32 \mathrm{t}$ & HD Euro V - 2008 Standards & 0 & 1386 & 1060 & 2830 & 3380 & 5039 & 6394 \\
\hline Heavy Duty Trucks & Rigid $>32 t$ & HD Euro VI & 0 & 0 & 0 & 0 & 0 & 0 & 1591 \\
\hline Heavy Duty Trucks & Articulated $14-20 \mathrm{t}$ & Conventional & 7592 & 5105 & 3685 & 5381 & 4116 & 4378 & 4381 \\
\hline Heavy Duty Trucks & Articulated $14-20 \mathrm{t}$ & HD Euro I - 91/542/EEC Stage I & 1320 & 890 & 645 & 942 & 739 & 803 & 830 \\
\hline Heavy Duty Trucks & Articulated $14-20 t$ & HD Euro II - 91/542/EEC Stage II & 1798 & 1219 & 883 & 1295 & 1012 & 1104 & 1141 \\
\hline Heavy Duty Trucks & Articulated $14-20 t$ & HD Euro III - 2000 Standards & 2884 & 1966 & 1427 & 2099 & 1648 & 1803 & 1869 \\
\hline Heavy Duty Trucks & Articulated $14-20 \mathrm{t}$ & HD Euro IV - 2005 Standards & 1668 & 1544 & 1109 & 1888 & 1724 & 2143 & 2225 \\
\hline Heavy Duty Trucks & Articulated $14-20 \mathrm{t}$ & HD Euro V - 2008 Standards & 0 & 356 & 246 & 619 & 746 & 1124 & 1440 \\
\hline Heavy Duty Trucks & Articulated $14-20 \mathrm{t}$ & HD Euro VI & 0 & 0 & 0 & 0 & 0 & 0 & 356 \\
\hline Heavy Duty Trucks & Articulated $20-28 \mathrm{t}$ & Conventional & 4978 & 3344 & 2416 & 3527 & 2699 & 2869 & 2872 \\
\hline Heavy Duty Trucks & Articulated $20-28 \mathrm{t}$ & HD Euro I - 91/542/EEC Stage I & 863 & 584 & 421 & 619 & 484 & 525 & 544 \\
\hline Heavy Duty Trucks & Articulated $20-28 \mathrm{t}$ & HD Euro II - 91/542/EEC Stage II & 1180 & 797 & 579 & 847 & 663 & 726 & 749 \\
\hline Heavy Duty Trucks & Articulated $20-28 \mathrm{t}$ & HD Euro III - 2000 Standards & 1892 & 1289 & 934 & 1377 & 1082 & 1181 & 1223 \\
\hline Heavy Duty Trucks & Articulated $20-28 \mathrm{t}$ & HD Euro IV - 2005 Standards & 1091 & 1014 & 728 & 1235 & 1130 & 1405 & 1456 \\
\hline Heavy Duty Trucks & Articulated $20-28 \mathrm{t}$ & HD Euro V - 2008 Standards & 0 & 232 & 163 & 404 & 491 & 737 & 945 \\
\hline Heavy Duty Trucks & Articulated $20-28 \mathrm{t}$ & HD Euro VI & 0 & 0 & 0 & 0 & 0 & 0 & 233 \\
\hline Heavy Duty Trucks & Articulated $28-34 \mathrm{t}$ & Conventional & 3929 & 2640 & 1905 & 2783 & 2129 & 2266 & 2266 \\
\hline Heavy Duty Trucks & Articulated $28-34 \mathrm{t}$ & HD Euro I - 91/542/EEC Stage I & 681 & 461 & 332 & 486 & 380 & 417 & 430 \\
\hline Heavy Duty Trucks & Articulated $28-34 \mathrm{t}$ & HD Euro II - 91/542/EEC Stage II & 930 & 631 & 456 & 667 & 525 & 571 & 589 \\
\hline Heavy Duty Trucks & Articulated $28-34 \mathrm{t}$ & HD Euro III - 2000 Standards & 1491 & 1018 & 736 & 1084 & 854 & 934 & 965 \\
\hline Heavy Duty Trucks & Articulated $28-34 \mathrm{t}$ & HD Euro IV - 2005 Standards & 863 & 797 & 573 & 976 & 892 & 1108 & 1149 \\
\hline Heavy Duty Trucks & Articulated $28-34 \mathrm{t}$ & HD Euro V - 2008 Standards & 0 & 186 & 129 & 318 & 387 & 583 & 745 \\
\hline Heavy Duty Trucks & Articulated $28-34 \mathrm{t}$ & HD Euro VI & 0 & 0 & 0 & 0 & 0 & 0 & 184 \\
\hline Heavy Duty Trucks & Articulated $34-40 t$ & Conventional & 59865 & 40247 & 29046 & 42418 & 32465 & 34537 & 34534 \\
\hline Heavy Duty Trucks & Articulated $34-40 t$ & HD Euro I - 91/542/EEC Stage I & 10393 & 7032 & 5077 & 7433 & 5823 & 6340 & 6545 \\
\hline Heavy Duty Trucks & Articulated $34-40 \mathrm{t}$ & HD Euro II - 91/542/EEC Stage II & 14187 & 9614 & 6954 & 10195 & 7993 & 8714 & 9004 \\
\hline Heavy Duty Trucks & Articulated $34-40 \mathrm{t}$ & HD Euro III - 2000 Standards & 22761 & 15493 & 11247 & 16544 & 13010 & 14224 & 14735 \\
\hline Heavy Duty Trucks & Articulated $34-40 t$ & HD Euro IV - 2005 Standards & 13142 & 12180 & 8745 & 14879 & 13591 & 16892 & 17537 \\
\hline Heavy Duty Trucks & Articulated $34-40 \mathrm{t}$ & HD Euro V - 2008 Standards & 0 & 2814 & 1948 & 4874 & 5895 & 8869 & 11368 \\
\hline Heavy Duty Trucks & Articulated $34-40 t$ & HD Euro VI & 0 & 0 & 0 & 0 & 0 & 0 & 2814 \\
\hline Heavy Duty Trucks & Articulated $40-50 \mathrm{t}$ & Conventional & 281 & 186 & 135 & 198 & 152 & 162 & 160 \\
\hline Heavy Duty Trucks & Articulated $40-50 \mathrm{t}$ & HD Euro I - 91/542/EEC Stage I & 47 & 31 & 23 & 34 & 28 & 31 & 29 \\
\hline Heavy Duty Trucks & Articulated $40-50 \mathrm{t}$ & HD Euro II - 91/542/EEC Stage II & 68 & 46 & 32 & 47 & 38 & 42 & 41 \\
\hline Heavy Duty Trucks & Articulated $40-50 \mathrm{t}$ & HD Euro III - 2000 Standards & 104 & 74 & 52 & 77 & 62 & 66 & 70 \\
\hline Heavy Duty Trucks & Articulated $40-50 \mathrm{t}$ & HD Euro IV - 2005 Standards & 62 & 58 & 40 & 69 & 62 & 77 & 82 \\
\hline Heavy Duty Trucks & Articulated $40-50 \mathrm{t}$ & HD Euro V - 2008 Standards & 0 & 12 & 9 & 22 & 28 & 42 & 53 \\
\hline Heavy Duty Trucks & Articulated $40-50 \mathrm{t}$ & HD Euro VI & 0 & 0 & 0 & 0 & 0 & 0 & 12 \\
\hline Heavy Duty Trucks & Articulated $50-60 \mathrm{t}$ & Conventional & 114 & 77 & 57 & 82 & 62 & 66 & 65 \\
\hline Heavy Duty Trucks & Articulated $50-60 \mathrm{t}$ & HD Euro I - 91/542/EEC Stage I & 21 & 15 & 9 & 13 & 10 & 12 & 12 \\
\hline Heavy Duty Trucks & Articulated $50-60 \mathrm{t}$ & HD Euro II - 91/542/EEC Stage II & 26 & 19 & 14 & 22 & 14 & 15 & 16 \\
\hline Heavy Duty Trucks & Articulated $50-60 \mathrm{t}$ & HD Euro III - 2000 Standards & 42 & 31 & 23 & 30 & 24 & 27 & 29 \\
\hline Heavy Duty Trucks & Articulated $50-60 \mathrm{t}$ & HD Euro IV - 2005 Standards & 26 & 23 & 17 & 30 & 28 & 31 & 33 \\
\hline Heavy Duty Trucks & Articulated $50-60 \mathrm{t}$ & HD Euro V - 2008 Standards & 0 & 4 & 3 & 9 & 10 & 15 & 20 \\
\hline Heavy Duty Trucks & Articulated $50-60 \mathrm{t}$ & HD Euro VI & 0 & 0 & 0 & 0 & 0 & 0 & 4 \\
\hline
\end{tabular}


Kleidi Station

\begin{tabular}{|c|c|c|c|c|c|c|}
\hline Sector & Subsector & Technology & 2008 & 2009 & 2010 & 2011 \\
\hline Passenger Cars & Gasoline $0,8-1,4$ I & ECE 15/02 & 7800 & 2271 & 2343 & 0 \\
\hline Passenger Cars & Gasoline $0,8-1,4$ I & ECE 15/03 & 72071 & 34295 & 77325 & 58893 \\
\hline Passenger Cars & Gasoline $0,8-1,4$ I & ECE $15 / 04$ & 445387 & 229683 & 554933 & 445594 \\
\hline Passenger Cars & Gasoline $0,8-1,4$ I & PC Euro 1 - 91/441/EEC & 489875 & 266233 & 679823 & 578107 \\
\hline Passenger Cars & Gasoline $0,8-1,4$ I & PC Euro 2 - 94/12/EEC & 791830 & 444318 & 1177693 & 1045644 \\
\hline Passenger Cars & Gasoline $0,8-1,4$ I & PC Euro 3 - 98/69/EC Stage2000 & 829366 & 500540 & 1349556 & 1223471 \\
\hline Passenger Cars & Gasoline $0,8-1,4$ I & 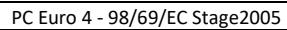 & 162369 & 117883 & 464622 & 523003 \\
\hline Passenger Cars & Gasoline $0,8-1,4$ I & PC Euro 5 - EC 715/2007 & 0 & 0 & 132705 & 220098 \\
\hline Passenger Cars & Gasoline 1,4-2,0 I & ECE 15/02 & 1126 & 292 & 278 & 0 \\
\hline Passenger Cars & Gasoline 1,4-2,0 I & ECE 15/03 & 16305 & 7438 & 16725 & 12801 \\
\hline Passenger Cars & Gasoline 1,4-2,01 & ECE 15/04 & 116551 & 57097 & 134970 & 105859 \\
\hline Passenger Cars & Gasoline 1,4 - 2,0 I & PC Euro 1 - 91/441/EEC & 208724 & 109741 & 279532 & 236972 \\
\hline Passenger Cars & Gasoline 1,4-2,0 I & PC Euro 2 - 94/12/EEC & 323849 & 175632 & 463802 & 409864 \\
\hline Passenger Cars & Gasoline 1,4-2,0 I & PC Euro 3 - 98/69/EC Stage2000 & 534904 & 317082 & 854180 & 773425 \\
\hline Passenger Cars & Gasoline $1,4-2,0$ I & PC Euro 4 - 98/69/EC Stage2005 & 121636 & 86866 & 294757 & 308125 \\
\hline Passenger Cars & Gasoline 1,4-2,0 I & PC Euro 5 - EC 715/2007 & 0 & 0 & 54313 & 88789 \\
\hline Passenger Cars & Gasoline $>2,0$ I & ECE 15/02 & 552 & 190 & 259 & 0 \\
\hline Passenger Cars & Gasoline $>2,0 \mathrm{I}$ & ECE 15/03 & 1055 & 518 & 1345 & 1176 \\
\hline Passenger Cars & Gasoline $>2,0 \mathrm{l}$ & ECE 15/04 & 2088 & 1032 & 2700 & 2378 \\
\hline Passenger Cars & Gasoline $>2,0 \mathrm{l}$ & PC Euro 1 - 91/441/EEC & 5943 & 2952 & 7758 & 6866 \\
\hline Passenger Cars & Gasoline $>2,0$ I & PC Euro 2 - 94/12/EEC & 8630 & 4316 & 11426 & 10179 \\
\hline Passenger Cars & Gasoline $>2,0$ I & PC Euro 3 - 98/69/EC Stage2000 & 36797 & 21005 & 56078 & 50369 \\
\hline Passenger Cars & Gasoline $>2,0$ I & PC Euro 4 - 98/69/EC Stage2005 & 12022 & 8226 & 24551 & 23630 \\
\hline Passenger Cars & Gasoline $>2,0 \mathrm{I}$ & PC Euro 5 - EC 715/2007 & 0 & 0 & 2287 & 3557 \\
\hline Passenger Cars & Diesel 1,4-2,0 I & Conventional & 1541 & 564 & 821 & 375 \\
\hline Passenger Cars & Diesel $1,4-2,0$ I & PC Euro 1 - 91/441/EEC & 8919 & 4374 & 8726 & 5611 \\
\hline Passenger Cars & Diesel $1,4-2,0$ I & PC Euro 2 - 94/12/EEC & 7974 & 3973 & 8047 & 5245 \\
\hline Passenger Cars & Diesel $1,4-2,0$ I & PC Euro 3 - 98/69/EC Stage2000 & 23381 & 14356 & 35259 & 28483 \\
\hline Passenger Cars & Diesel $1,4-2,0 \mid$ & PC Euro 4 - 98/69/EC Stage2005 & 3481 & 2667 & 13588 & 16947 \\
\hline Passenger Cars & Diesel 1,4 - 2,0 I & PC Euro 5 - EC 715/2007 & 0 & 0 & 6021 & 10334 \\
\hline Passenger Cars & Diesel $>2,0$ I & Conventional & 173 & 44 & 42 & 11 \\
\hline Passenger Cars & Diesel $>2,0 \mathrm{I}$ & PC Euro 1 - 91/441/EEC & 2342 & 1044 & 1984 & 1170 \\
\hline Passenger Cars & Diesel $>2,0 \mathrm{I}$ & PC Euro 2 - 94/12/EEC & 1952 & 894 & 1734 & 1033 \\
\hline Passenger Cars & Diesel $>2,0 \mathrm{I}$ & PC Euro 3 - 98/69/EC Stage2000 & 8674 & 5260 & 13663 & 11698 \\
\hline Passenger Cars & Diesel $>2,0$ I & 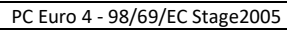 & 1681 & 1245 & 4888 & 5582 \\
\hline Passenger Cars & Diesel $>2,0 \mathrm{I}$ & PC Euro 5 - EC 715/2007 & 0 & 0 & 1402 & 2431 \\
\hline Passenger Cars & LPG & Conventional & 2 & 0 & 0 & 0 \\
\hline Passenger Cars & LPG & PC Euro 1 - 91/441/EEC & 29 & 4 & 3 & 0 \\
\hline Passenger Cars & LPG & PC Euro 2 - 94/12/EEC & 495 & 192 & 292 & 122 \\
\hline Passenger Cars & LPG & PC Euro 3 - 98/69/EC Stage2000 & 1981 & 1177 & 2950 & 2395 \\
\hline Passenger Cars & LPG & 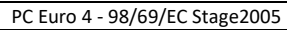 & 368 & 273 & 1059 & 1212 \\
\hline Passenger Cars & LPG & PC Euro 5 - EC 715/2007 & 0 & 0 & 297 & 524 \\
\hline Passenger Cars & 2-Stroke & Conventional & 0 & 0 & 58598 & 79533 \\
\hline Passenger Cars & Hybrid Gasoline 1,4 - 2,0I & 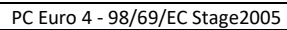 & 0 & 0 & 66888 & 91013 \\
\hline Heavy Duty Trucks & Rigid $<=7,5 \mathrm{t}$ & Conventional & 131163 & 16769 & 127032 & 131158 \\
\hline Heavy Duty Trucks & Rigid $<=7,5 \mathrm{t}$ & HD Euro I - 91/542/EEC Stage I & 58145 & 7441 & 56481 & 59120 \\
\hline Heavy Duty Trucks & Rigid $<=7,5 \mathrm{t}$ & HD Euro II - 91/542/EEC Stage II & 95473 & 12218 & 92731 & 97069 \\
\hline Heavy Duty Trucks & Rigid $<=7,5 \mathrm{t}$ & HD Euro III - 2000 Standards & 108511 & 13886 & 105399 & 110324 \\
\hline Heavy Duty Trucks & Rigid $<=7,5 \mathrm{t}$ & HD Euro IV - 2005 Standards & 44426 & 7737 & 59712 & 75098 \\
\hline Heavy Duty Trucks & Rigid $<=7,5 \mathrm{t}$ & HD Euro V - 2008 Standards & 0 & 1791 & 14492 & 27772 \\
\hline Heavy Duty Trucks & Rigid $<=7,5 \mathrm{t}$ & HD Euro VI & 0 & 0 & 0 & 0 \\
\hline Heavy Duty Trucks & Rigid $7,5-12 \mathrm{t}$ & Conventional & 59008 & 7544 & 57148 & 59009 \\
\hline Heavy Duty Trucks & Rigid $7,5-12 \mathrm{t}$ & HD Euro I - 91/542/EEC Stage I & 26160 & 3347 & 25411 & 26598 \\
\hline Heavy Duty Trucks & Rigid $7,5-12 \mathrm{t}$ & HD Euro II - 91/542/EEC Stage II & 42950 & 5497 & 41721 & 43670 \\
\hline Heavy Duty Trucks & Rigid $7,5-12 \mathrm{t}$ & HD Euro III - 2000 Standards & 48817 & 6247 & 47420 & 49637 \\
\hline Heavy Duty Trucks & Rigid $7,5-12 \mathrm{t}$ & HD Euro IV - 2005 Standards & 19986 & 3481 & 26864 & 33788 \\
\hline Heavy Duty Trucks & Rigid $7,5-12 \mathrm{t}$ & HD Euro V - 2008 Standards & 0 & 806 & 6519 & 12494 \\
\hline Heavy Duty Trucks & Rigid $7,5-12 \mathrm{t}$ & HD Euro VI & 0 & 0 & 0 & 0 \\
\hline Heavy Duty Trucks & Rigid $12-14 \mathrm{t}$ & Conventional & 16510 & 2111 & 15991 & 16507 \\
\hline Heavy Duty Trucks & Rigid $12-14 \mathrm{t}$ & HD Euro I - 91/542/EEC Stage I & 7317 & 937 & 7106 & 7442 \\
\hline Heavy Duty Trucks & Rigid $12-14 t$ & HD Euro II - 91/542/EEC Stage II & 12015 & 1538 & 11671 & 12217 \\
\hline Heavy Duty Trucks & Rigid $12-14 t$ & HD Euro III - 2000 Standards & 13657 & 1748 & 13267 & 13883 \\
\hline Heavy Duty Trucks & Rigid $12-14 \mathrm{t}$ & HD Euro IV - 2005 Standards & 5592 & 974 & 7517 & 9452 \\
\hline Heavy Duty Trucks & Rigid $12-14 t$ & HD Euro V - 2008 Standards & 0 & 225 & 1824 & 3497 \\
\hline Heavy Duty Trucks & Rigid $12-14 \mathrm{t}$ & HD Euro VI & 0 & 0 & 0 & 0 \\
\hline Heavy Duty Trucks & Rigid $14-20 t$ & Conventional & 45471 & 5814 & 44040 & 45471 \\
\hline Heavy Duty Trucks & Rigid $14-20 t$ & HD Euro I - 91/542/EEC Stage I & 20158 & 2580 & 19581 & 20495 \\
\hline Heavy Duty Trucks & Rigid $14-20 t$ & HD Euro II - 91/542/EEC Stage II & 33097 & 4236 & 32147 & 33653 \\
\hline Heavy Duty Trucks & Rigid $14-20 t$ & HD Euro III - 2000 Standards & 37618 & 4815 & 36541 & 38250 \\
\hline Heavy Duty Trucks & Rigid $14-20 t$ & HD Euro IV - 2005 Standards & 15403 & 2682 & 20698 & 26032 \\
\hline Heavy Duty Trucks & Rigid $14-20 t$ & HD Euro V - 2008 Standards & 0 & 621 & 5026 & 9630 \\
\hline Heavy Duty Trucks & Rigid $14-20 t$ & HD Euro VI & 0 & 0 & 0 & 0 \\
\hline Heavy Duty Trucks & Rigid $20-26 \mathrm{t}$ & Conventional & 36958 & 4725 & 35794 & 36959 \\
\hline Heavy Duty Trucks & Rigid $20-26 \mathrm{t}$ & HD Euro I - 91/542/EEC Stage I & 16385 & 2097 & 15911 & 16661 \\
\hline Heavy Duty Trucks & Rigid $20-26 \mathrm{t}$ & HD Euro II - 91/542/EEC Stage II & 26898 & 3442 & 26129 & 27354 \\
\hline Heavy Duty Trucks & Rigid $20-26 \mathrm{t}$ & HD Euro III - 2000 Standards & 30572 & 3913 & 29696 & 31084 \\
\hline Heavy Duty Trucks & Rigid $20-26 \mathrm{t}$ & HD Euro IV - 2005 Standards & 12519 & 2180 & 16823 & 21159 \\
\hline Heavy Duty Trucks & Rigid $20-26 \mathrm{t}$ & HD Euro V - 2008 Standards & 0 & 504 & 4086 & 7823 \\
\hline Heavy Duty Trucks & Rigid $20-26 \mathrm{t}$ & HD Euro VI & 0 & 0 & 0 & 0 \\
\hline Heavy Duty Trucks & Rigid $26-28 \mathrm{t}$ & Conventional & 94 & 12 & 91 & 98 \\
\hline Heavy Duty Trucks & Rigid $26-28 \mathrm{t}$ & HD Euro I - 91/542/EEC Stage I & 42 & 5 & 40 & 43 \\
\hline Heavy Duty Trucks & Rigid $26-28 \mathrm{t}$ & HD Euro II - 91/542/EEC Stage II & 68 & 9 & 68 & 74 \\
\hline Heavy Duty Trucks & Rigid $26-28 \mathrm{t}$ & HD Euro III - 2000 Standards & 78 & 10 & 74 & 80 \\
\hline Heavy Duty Trucks & Rigid $26-28 \mathrm{t}$ & HD Euro IV - 2005 Standards & 31 & 6 & 46 & 55 \\
\hline Heavy Duty Trucks & Rigid $26-28 \mathrm{t}$ & HD Euro V - 2008 Standards & 0 & 1 & 11 & 18 \\
\hline Heavy Duty Trucks & Rigid $26-28 \mathrm{t}$ & HD Euro VI & 0 & 0 & 0 & 0 \\
\hline Heavy Duty Trucks & Rigid $28-32 \mathrm{t}$ & Conventional & 3461 & 442 & 3351 & 3460 \\
\hline Heavy Duty Trucks & Rigid $28-32 \mathrm{t}$ & HD Euro I - 91/542/EEC Stage I & 1533 & 196 & 1487 & 1561 \\
\hline Heavy Duty Trucks & Rigid $28-32 \mathrm{t}$ & HD Euro II - 91/542/EEC Stage II & 2520 & 322 & 2445 & 2563 \\
\hline Heavy Duty Trucks & Rigid $28-32 \mathrm{t}$ & HD Euro III - 2000 Standards & 2863 & 367 & 2781 & 2913 \\
\hline Heavy Duty Trucks & Rigid $28-32 \mathrm{t}$ & HD Euro IV - 2005 Standards & 1174 & 204 & 1573 & 1979 \\
\hline
\end{tabular}




\begin{tabular}{|c|c|c|c|c|c|c|c|c|c|}
\hline Sector & Subsector & Technology & 2008 & 2009 & 2010 & 2011 & 2012 & 2013 & 2014 \\
\hline Heavy Duty Trucks & Rigid $28-32 \mathrm{t}$ & HD Euro V - 2008 Standards & 0 & 47 & 382 & 731 & 982 & 1071 & 1201 \\
\hline Heavy Duty Trucks & Rigid $28-32 \mathrm{t}$ & HD Euro VI & 0 & 0 & 0 & 0 & 0 & 0 & 297 \\
\hline Heavy Duty Trucks & Rigid $>32 \mathrm{t}$ & Conventional & 19087 & 2441 & 18487 & 19088 & 16228 & 12447 & 10864 \\
\hline Heavy Duty Trucks & Rigid $>32 t$ & HD Euro I - 91/542/EEC Stage I & 8465 & 1083 & 8218 & 8604 & 7537 & 5962 & 5434 \\
\hline Heavy Duty Trucks & Rigid $>32 t$ & HD Euro II - 91/542/EEC Stage II & 13896 & 1778 & 13495 & 14129 & 12373 & 9790 & 8921 \\
\hline Heavy Duty Trucks & Rigid $>32 \mathrm{t}$ & HD Euro III - 2000 Standards & 15793 & 2021 & 15341 & 16058 & 14065 & 11128 & 10139 \\
\hline Heavy Duty Trucks & Rigid $>32 t$ & HD Euro IV - 2005 Standards & 6465 & 1126 & 8691 & 10933 & 11292 & 10278 & 9362 \\
\hline Heavy Duty Trucks & Rigid $>32 t$ & HD Euro V - 2008 Standards & 0 & 261 & 2109 & 4044 & 5424 & 5898 & 6630 \\
\hline Heavy Duty Trucks & Rigid $>32 t$ & HD Euro VI & 0 & 0 & 0 & 0 & 0 & 0 & 1650 \\
\hline Heavy Duty Trucks & Articulated $14-20 \mathrm{t}$ & Conventional & 7592 & 961 & 7329 & 7688 & 6605 & 5125 & 4543 \\
\hline Heavy Duty Trucks & Articulated $14-20 t$ & HD Euro I - 91/542/EEC Stage I & 1320 & 168 & 1282 & 1346 & 1187 & 940 & 861 \\
\hline Heavy Duty Trucks & Articulated $14-20 t$ & HD Euro II - 91/542/EEC Stage II & 1798 & 230 & 1755 & 1850 & 1625 & 1293 & 1184 \\
\hline Heavy Duty Trucks & Articulated $14-20 \mathrm{t}$ & HD Euro III - 2000 Standards & 2884 & 370 & 2838 & 2999 & 2645 & 2111 & 1939 \\
\hline Heavy Duty Trucks & Articulated $14-20 \mathrm{t}$ & HD Euro IV - 2005 Standards & 1668 & 291 & 2205 & 2698 & 2767 & 2508 & 2308 \\
\hline Heavy Duty Trucks & Articulated $14-20 t$ & HD Euro V - 2008 Standards & 0 & 67 & 490 & 885 & 1198 & 1315 & 1493 \\
\hline Heavy Duty Trucks & Articulated $14-20 t$ & HD Euro VI & 0 & 0 & 0 & 0 & 0 & 0 & 369 \\
\hline Heavy Duty Trucks & Articulated $20-28 \mathrm{t}$ & Conventional & 4978 & 630 & 4804 & 5039 & 4331 & 3358 & 2978 \\
\hline Heavy Duty Trucks & Articulated $20-28 \mathrm{t}$ & HD Euro I - 91/542/EEC Stage I & 863 & 110 & 838 & 885 & 776 & 615 & 564 \\
\hline Heavy Duty Trucks & Articulated $20-28 \mathrm{t}$ & HD Euro II - 91/542/EEC Stage II & 1180 & 150 & 1151 & 1211 & 1065 & 850 & 776 \\
\hline Heavy Duty Trucks & Articulated $20-28 \mathrm{t}$ & HD Euro III - 2000 Standards & 1892 & 243 & 1858 & 1967 & 1736 & 1383 & 1268 \\
\hline Heavy Duty Trucks & Articulated $20-28 \mathrm{t}$ & HD Euro IV - 2005 Standards & 1091 & 191 & 1447 & 1764 & 1814 & 1645 & 1510 \\
\hline Heavy Duty Trucks & Articulated $20-28 \mathrm{t}$ & HD Euro V - 2008 Standards & 0 & 44 & 325 & 578 & 788 & 863 & 980 \\
\hline Heavy Duty Trucks & Articulated $20-28 \mathrm{t}$ & HD Euro VI & 0 & 0 & 0 & 0 & 0 & 0 & 242 \\
\hline Heavy Duty Trucks & Articulated $28-34 \mathrm{t}$ & Conventional & 3929 & 497 & 3790 & 3976 & 3416 & 2653 & 2350 \\
\hline Heavy Duty Trucks & Articulated $28-34 \mathrm{t}$ & HD Euro I - 91/542/EEC Stage I & 681 & 87 & 661 & 694 & 610 & 488 & 445 \\
\hline Heavy Duty Trucks & Articulated $28-34 \mathrm{t}$ & HD Euro II - 91/542/EEC Stage II & 930 & 119 & 906 & 953 & 843 & 669 & 611 \\
\hline Heavy Duty Trucks & Articulated $28-34 \mathrm{t}$ & HD Euro III - 2000 Standards & 1491 & 192 & 1465 & 1549 & 1370 & 1094 & 1001 \\
\hline Heavy Duty Trucks & Articulated $28-34 \mathrm{t}$ & HD Euro IV - 2005 Standards & 863 & 150 & 1140 & 1395 & 1431 & 1297 & 1192 \\
\hline Heavy Duty Trucks & Articulated $28-34 \mathrm{t}$ & HD Euro V - 2008 Standards & 0 & 35 & 256 & 455 & 621 & 682 & 772 \\
\hline Heavy Duty Trucks & Articulated $28-34 \mathrm{t}$ & HD Euro VI & 0 & 0 & 0 & 0 & 0 & 0 & 191 \\
\hline Heavy Duty Trucks & Articulated $34-40 \mathrm{t}$ & Conventional & 59865 & 7579 & 57769 & 60601 & 52105 & 40429 & 35812 \\
\hline Heavy Duty Trucks & Articulated $34-40 t$ & HD Euro I - 91/542/EEC Stage I & 10393 & 1324 & 10098 & 10619 & 9345 & 7421 & 6787 \\
\hline Heavy Duty Trucks & Articulated $34-40 \mathrm{t}$ & HD Euro II - 91/542/EEC Stage II & 14187 & 1810 & 13831 & 14565 & 12828 & 10201 & 9337 \\
\hline Heavy Duty Trucks & Articulated $34-40 \mathrm{t}$ & HD Euro III - 2000 Standards & 22761 & 2917 & 22368 & 23636 & 20881 & 16651 & 15280 \\
\hline Heavy Duty Trucks & Articulated $34-40 t$ & HD Euro IV - 2005 Standards & 13142 & 2294 & 17393 & 21257 & 21813 & 19774 & 18186 \\
\hline Heavy Duty Trucks & Articulated $34-40 \mathrm{t}$ & HD Euro V - 2008 Standards & 0 & 530 & 3875 & 6963 & 9462 & 10382 & 11789 \\
\hline Heavy Duty Trucks & Articulated $34-40 t$ & HD Euro VI & 0 & 0 & 0 & 0 & 0 & 0 & 2919 \\
\hline Heavy Duty Trucks & Articulated $40-50 \mathrm{t}$ & Conventional & 281 & 35 & 268 & 283 & 244 & 190 & 165 \\
\hline Heavy Duty Trucks & Articulated $40-50 \mathrm{t}$ & HD Euro I - 91/542/EEC Stage I & 47 & 6 & 46 & 49 & 44 & 36 & 30 \\
\hline Heavy Duty Trucks & Articulated $40-50 \mathrm{t}$ & HD Euro II - 91/542/EEC Stage II & 68 & 9 & 63 & 68 & 61 & 50 & 42 \\
\hline Heavy Duty Trucks & Articulated $40-50 \mathrm{t}$ & HD Euro III - 2000 Standards & 104 & 14 & 103 & 111 & 100 & 77 & 72 \\
\hline Heavy Duty Trucks & Articulated $40-50 \mathrm{t}$ & HD Euro IV - 2005 Standards & 62 & 11 & 80 & 98 & 100 & 90 & 85 \\
\hline Heavy Duty Trucks & Articulated $40-50 \mathrm{t}$ & HD Euro V - 2008 Standards & 0 & 2 & 17 & 31 & 44 & 50 & 55 \\
\hline Heavy Duty Trucks & Articulated $40-50 \mathrm{t}$ & HD Euro VI & 0 & 0 & 0 & 0 & 0 & 0 & 13 \\
\hline Heavy Duty Trucks & Articulated $50-60 \mathrm{t}$ & Conventional & 114 & 15 & 114 & 117 & 100 & 77 & 68 \\
\hline Heavy Duty Trucks & Articulated $50-60 \mathrm{t}$ & HD Euro I - 91/542/EEC Stage I & 21 & 3 & 17 & 18 & 17 & 14 & 13 \\
\hline Heavy Duty Trucks & Articulated $50-60 \mathrm{t}$ & HD Euro II - 91/542/EEC Stage II & 26 & 4 & 28 & 31 & 22 & 18 & 17 \\
\hline Heavy Duty Trucks & Articulated $50-60 \mathrm{t}$ & HD Euro III - 2000 Standards & 42 & 6 & 46 & 43 & 39 & 32 & 30 \\
\hline Heavy Duty Trucks & Articulated $50-60 \mathrm{t}$ & HD Euro IV - 2005 Standards & 26 & 4 & 34 & 43 & 44 & 36 & 34 \\
\hline Heavy Duty Trucks & Articulated $50-60 \mathrm{t}$ & HD Euro V - 2008 Standards & 0 & 1 & 6 & 12 & 17 & 18 & 21 \\
\hline Heavy Duty Trucks & Articulated $50-60 \mathrm{t}$ & HD Euro VI & 0 & 0 & 0 & 0 & 0 & 0 & 4 \\
\hline
\end{tabular}

